\title{
HOW BAD OR GOOD ARE THE EXTERNAL FORWARD SHOCK AFTERGLOW MODELS OF GAMMA-RAY BURSTS?
}

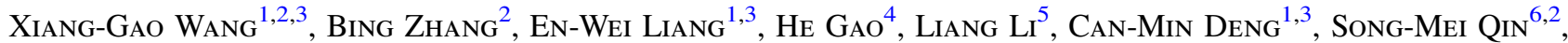 \\ Qing-Wen Tang ${ }^{7}$, D. Alexander Kann ${ }^{8}$, Felix Ryde ${ }^{9}$, and Pawan Kumar ${ }^{10}$ \\ ${ }^{1}$ GXU-NAOC Center for Astrophysics and Space Sciences, Department of Physics, Guangxi University, \\ Nanning 530004, China; wangxg@gxu.edu.cn, lew@gxu.edu.cn \\ ${ }^{2}$ Department of Physics and Astronomy, University of Nevada Las Vegas, NV 89154, USA; zhang@ physics.unlv.edu \\ ${ }^{3}$ Guangxi Key Laboratory for the Relativistic Astrophysics, Nanning 530004, China \\ ${ }^{4}$ Department of Astronomy and Astrophysics, Pennsylvania State University, 525 Davey Laboratory, University Park, PA 16802, USA \\ ${ }^{5}$ Department of Physics, Stockholm University, SE-106 91 Stockholm, Sweden \\ ${ }^{6}$ Mathematics and Physics Section, Guangxi University of Chinese Medicine, Nanning 53001, China \\ ${ }^{7}$ School of Astronomy and Space Science, Nanjing University, Nanjing, Jiangsu 210093, China \\ ${ }^{8}$ Thüringer Landessternwarte Tautenburg, Sternwarte 5, D-07778 Tautenburg, Germany \\ ${ }^{9}$ Department of Astronomy, University of Texas at Austin, Austin, TX 78712, USA \\ ${ }^{10}$ Department of Physics, KTH Royal Institute of Technology, SE-106 91 Stockholm, Sweden \\ Received 2015 March 11; accepted 2015 June 2; published 2015 July 16
}

\begin{abstract}
The external forward shock models have been the standard paradigm to interpret the broadband afterglow data of gamma-ray bursts (GRBs). One prediction of the models is that some afterglow temporal breaks at different energy bands should be achromatic; that is, the break times should be the same in different frequencies. Multiwavelength observations in the Swift era have revealed chromatic afterglow behaviors at least in some GRBs, casting doubts on the external forward shock origin of GRB afterglows. In this paper, using a large sample of GRBs with both X-ray and optical afterglow data, we perform a systematic study to address the question: how bad or good are the external forward shock models? Our sample includes 85 GRBs up to 2014 March with well-monitored X-ray and optical light curves. Based on how well the data abide by the external forward shock models, we categorize them into five grades and three samples. The first two grades (Grade I and II) include 45 of 85 GRBs. They show evidence of, or are consistent with having, an achromatic break. The temporal and spectral behaviors in each afterglow segment are consistent with the predictions (the "closure relations") of the forward shock models. These GRBs are included in the Gold sample. The next two grades (Grade III and IV) include 37 of 85 GRBs. They are also consistent with having an achromatic break, even though one or more afterglow segments do not comply with the closure relations. These GRBs are included in the Silver sample. Finally, Grade V (3/85) shows direct evidence of chromatic behaviors, suggesting that the external shock models are inconsistent with the data. These are included in the Bad sample. We further perform statistical analyses of various observational properties (temporal index $\alpha$, spectral index $\beta$, break time $t_{\mathrm{b}}$ ) and model parameters (energy injection index $q$, electron spectral index $p$, jet opening angle $\theta_{j}$, radiative efficiency $\eta_{\gamma}$, and so on) of the GRBs in the Gold sample, and derive constraints on the magnetization parameter $\epsilon_{B}$ in the forward shock. Overall, we conclude that the simplest external forward shock models can account for the multiwavelength afterglow data of at least half of the GRBs. When more advanced modeling (e.g., long-lasting reverse shock, structured jets, arbitrary circumburst medium density profile) is invoked, up to $>90 \%$ of the afterglows may be interpreted within the framework of the external shock models.
\end{abstract}

Key words: methods: statistical - gamma-ray burst: general - radiation mechanisms: nonthermal

\section{INTRODUCTION}

Gamma-ray bursts (GRBs) are the most luminous explosions in the universe. They signify the birth of a stellar-mass black hole or a rapidly rotating magnetized neutron star during core collapses of massive stars or mergers of compact objects (see Kumar \& Zhang 2015 for a recent review).

Multiwavelength GRB afterglows were predicted (Mészáros \& Rees 1997) before their first discoveries (Costa et al. 1997; Frail et al. 1997; van Paradijs et al. 1997). This was based on a generic external forward shock model. Regardless of the physical nature of the progenitor and central engine, a relativistic jet is launched, which is decelerated by a circumburst medium by a pair of external (forward and reverse) shocks. The reverse shock is likely short-lived. The forward shock, on the other hand, continues to plow into the medium as the jet is decelerated. The synchrotron radiation of electrons accelerated from the external forward shock powers the broadband electromagnetic radiation with a decreasing amplitude. This is the broadband afterglow of GRBs (Mészáros \& Rees 1997; Mészáros et al. 1998; Sari et al. 1998, 1999; Rhoads 1999; Chevalier \& Li 2000).

Before 2004, the observations of the broadband, late-time afterglow emission of GRBs generally show broken power-law (BPL) light curves and instantaneous spectra. Detailed studies (e.g., Waxman 1997; Wijers et al. 1997; Harrison et al. 1999; Huang et al. 1999, 2000; Wijers \& Galama 1999; Panaitescu \& Kumar 2001, 2002; Yost et al. 2003; Wu et al. 2004) suggested that these late-time data are generally consistent with the predictions of the external forward shock models.

The launch of the Swift satellite in 2004 (Gehrels et al. 2004) allowed systematic observations of the multiwavelength GRB afterglow at early epochs. These data, especially the early $\mathrm{X}$-ray afterglow data, presented surprises to modelers. The overall X-ray light curves include five distinct temporal components (Zhang et al. 2006): I: an early-time steep decay 
phase connected to the prompt emission (Barthelmy et al. 2005; Tagliaferri et al. 2005; Zhang et al. 2007c); II: a shallow decay (or plateau) phase, which may signify continuous injection of energy into the blast wave (Nousek et al. 2006; Zhang et al. 2006; Liang et al. 2007); III: a normal decay phase consistent with the forward shock emission of a constantenergy fireball; IV: a late, steep decay phase likely due to a jet break origin (e.g., Liang et al. 2008; Racusin et al. 2009); and $\mathrm{V}$ : erratic X-ray flares, likely powered by late central engine activities (Burrows et al. 2005; Ioka et al. 2005; Fan \& Wei 2005; Liang et al. 2006; Zhang et al. 2006; Chincarini et al. 2007; Lazzati \& Perna 2007; Maxham \& Zhang 2009; Margutti et al. 2010). Components I and V are believed to have an internal origin (in contrast to the external shock origin). The other three components (II, III, and IV) may be interpreted within the framework of the external shock models.

The optical-afterglow light curves also show interesting temporal behaviors (Kann et al. 2006, 2010, 2011; Liang et al. 2006, 2013; Nardini et al. 2006; Panaitescu \& Vestrand 2008, 2011; Li et al. 2012; Wang et al. 2013; Yi et al. 2013). In a spirit similar to Zhang et al. (2006), Li et al. (2012) attempted to summarize a "synthetic" light curve of optical emission. They found more components with distinct physical origins: Ia: prompt optical flares; Ib: an early optical flare of an external reverse shock origin; II: an early shallowdecay segment; III: the standard afterglow component (the normal decay component, sometimes with an early onset hump); IV: the post-jet-break phase; V: late optical flares; VI: late rebrightening humps; and VII: late supernova (SN) bumps. The components II, III, and IV find their counterparts in the canonical X-ray light curve (components II, III, and IV in Zhang et al. 2006). Some flares in the optical band have counterparts in X-rays, but some others do not (Swenson et al. 2013). Some components (e.g., the reverse shock component $\mathrm{Ib}$ and the $\mathrm{SN}$ component VII) are unique to the optical band only.

There are two types of temporal breaks in the external shock models. One type corresponds to the crossing of a characteristic frequency in the observational band (Sari et al. 1998). Such spectrally related breaks occur at different epochs in different energy bands and therefore are chromatic. A testable feature of such a break is that the spectral indices before and after the temporal break should be distinctly different. The second type of breaks is related to the hydrodynamic or geometric properties of the system. Since both effects affect the global behavior of the blast wave, these breaks should be achromatic: the temporal breaks in different energy bands should occur around the same observational time.

Most observed breaks in the GRB light curves are likely of a hydrodynamic or geometric origin. Observationally, essentially all of the temporal breaks observed in the X-ray light curves are consistent with having no spectral changes across the break times (Liang et al. 2007, 2008). Theoretically, the spectral breaks, especially the cooling break, are predicted to be very smooth and are barely observable from the data (Uhm \& Zhang 2014a, see also Granot \& Sari 2002; van Eerten \& Wijers 2009). As a result, one expects that the temporal breaks seen by Swift should be strictly achromatic based on the external forward shock models.

Broadband afterglow data of GRBs are rapidly accumulating. Shortly after Swift detected early X-ray afterglow light curves of GRBs, some authors noticed that the basic requirement of achromaticity of GRB afterglows is violated at least in some GRBs (e.g., Fan \& Piran 2006; Panaitescu et al. 2006; Huang et al. 2007; Liang et al. 2007, 2008). In particular, while a significant break is seen in the X-ray light curves of some GRBs, the optical light curve does not show evidence of a break at the corresponding time (e.g., Molinari et al. 2007; Troja et al. 2007). Such a puzzling effect led theorists to suggest various non-forward-shock models of the X-ray afterglow: the long-lasting reverse shock model (Genet et al. 2007; Uhm \& Beloborodov 2007), the dust scattering model (Shao \& Dai 2007), and the long-lasting central engine model (Ghisellini et al. 2007; Kumar et al. 2008a, 2008b).

Indeed, if most GRB afterglows are chromatic, one must throw away the standard forward shock paradigm and probably point to other factors, in particular, the long-lasting central engine, to account for the X-ray afterglow. This would have profound implications for our understanding of the GRB central engine and emission physics. Yet, there seem to exist some GRBs (e.g., the latest bright GRB 130427A) whose multiwavelength data are consistent with the simplest forward shock afterglow model (e.g., Maselli et al. 2014; Perley et al. 2014).

It is therefore natural to ask the following question: in general how bad or how good are the external forward shock models in interpreting the GRB afterglow data?

This paper aims to address this question through a systematic data analysis and theoretical modeling of a large sample of multiwavelength afterglows. We study a sample of 85 Swift GRBs up to 2014 March, which all have high-quality X-ray and optical light curve data to allow us to study the compliance of the data with the external forward shock models. The sample selection and data analyses are described in Section 2. The theoretical external forward shock model and, in particular, the so-called closure relations are presented in Section 3. In Section 4, we grade the afterglows based on how well they abide by the forward shock models and categorize them into five grades and three samples. A statistical analysis of various observational and theoretical parameters for the Gold sample is presented in Section 5. Our results are summarized in Section 6 with some discussion. We notice that $\mathrm{Li}$ et al. (2015) recently carried out a similar analysis, with the focus on the consistency of the data with afterglow models in individual temporal segments of X-ray and optical light curves, without analyzing the global achromatic/chromatic behaviors of the afterglows.

Throughout the paper, the subscripts " $O$ " and " $X$ " denote the optical and X-ray bands, respectively, and the subscripts " 1 " and " 2 " denote the pre- and postbreak segments, respectively. In addition, two spectral regimes are defined: "I" for $\nu>\max \left(\nu_{\mathrm{m}}, \nu_{\mathrm{c}}\right)$, and "II" for $\nu_{\mathrm{m}}<\nu<\nu_{\mathrm{c}}$, where $\nu_{\mathrm{m}}$ and $\nu_{\mathrm{c}}$ are the minimum injection frequency and cooling frequency for synchrotron radiation, respectively.

\section{SAMPLE AND DATA}

We systematically investigate all of the Swift GRBs that have X-ray and optical afterglow data, over a span of almost 10 years from the launch of Swift to 2014 March. A sample of 260 optical light curves are compiled from published papers or GCN Circulars, and a sample of $\sim 900 \mathrm{X}$-ray light curves are obtained from the Swift XRT data archive. Well-sampled light curves in both the X-ray and optical bands are available for 99 GRBs. Fifteen GRBs do not have well-constrained spectral indices in either the optical or X-ray bands to allow us to 
perform some theoretical constraints (see details below). Fourteen of them are removed from the sample. GRB 070420 is the only GRB without adequate spectral information that is included in our sample. This is because it has a clear chromatic feature, which allows us to group it into the Bad sample even if the spectral information is not available (see details in Section 4.2). The remaining $84 \mathrm{GRBs}$ are included in our final sample, whose information is presented in Table 1. The optical afterglow data is available in GXU Astrophysics website: http://astro.gxu.edu.cn/data.html or the optical data, the correction due to Galactic extinction is taken into account using the reddening map presented by Schlegel et al. (1998). Due to large uncertainties, we do not make corrections to the extinction in the GRB host galaxies.

In order to quantify the rich temporal features of the GRB light curves, we fit the light curves with a model with multiple components. The basic component of our model is either a single power-law (SPL) function

$$
F_{1}=F_{01} t^{-\alpha}
$$

or a smooth BPL function

$$
F_{2}=F_{02}\left[\left(\frac{t}{t_{\mathrm{b}}}\right)^{\alpha_{1} \omega}+\left(\frac{t}{t_{\mathrm{b}}}\right)^{\alpha_{2} \omega}\right]^{-1 / \omega},
$$

where $\alpha, \alpha_{1}, \alpha_{2}$ are the temporal slopes, $t_{\mathrm{b}}$ is the break time, and $\omega$ measures the sharpness of the break. In some afterglow models, a double BPL light curve is expected. For example, it is theoretically expected that the afterglow light curve may have a shallow segment early on due to energy injection, then transits to a normal decay segment when energy injection is over, and finally steepens due to a jet break (e.g., in the canonical X-ray afterglow light curve; Zhang et al. 2006). We therefore also consider a smooth triple power-law (TPL) function to fit some light curves. In these cases, we extend Equation (2) (with $t_{\mathrm{b}}$ defined as $t_{\mathrm{b}, 1}$ ) to the following function (Liang et al. 2008):

$$
F_{3}=\left(F_{2}^{-\omega_{2}}+F_{4}^{-\omega_{2}}\right)^{-1 / \omega_{2}}
$$

where $\omega_{2}$ is the sharpness factor of the second break at $t_{\mathrm{b}, 2}$, and

$$
F_{4}=F_{2}\left(t_{\mathrm{b}, 2}\right)\left(\frac{t}{t_{\mathrm{b}, 2}}\right)^{-\alpha_{3}}
$$

We perform best fits to the data using a subroutine called MPFIT. ${ }^{11}$ The sharpness parameter $\omega$ is usually adopted as 3 or 1 in our fitting. The parameter $t_{\mathrm{b}}$ is not significantly affected by the choice of $\omega$, but the pre- and postbreak slopes (i.e., $\alpha_{1}$ and $\alpha_{2}$ ) somewhat depend on the value of $\omega$ (Liang et al. 2007). The larger the value of $\omega$, the sharper the break. The breaks in most X-ray and optical light curves at later times (e.g., the energy injection breaks and the jet breaks) can be well fit with $\omega=3$, which is consistent with the fit results using other empirical models (e.g., Willingale et al. 2007). Some very smooth breaks (e.g., the onset breaks in the early optical light curves) require $\omega$ being around one (Liang et al. 2007; Li et al. 2012), and we adopt this value when it is needed.

\footnotetext{
11 http://www.physics.wisc.edu/ craigm/idl/fitting.html.
}

One focus of our analysis is to study the "chromaticity" of the light curves in the X-ray and optical bands. In principle there are two approaches to this. The first approach is to blindly search for $t_{\mathrm{b}}$ using the best fits to the optical and X-ray data, respectively, and compare how different the two $t_{\mathrm{b}}$ values are. Such an approach usually gives different break times in the two bands (Liang et al. 2007, 2008; Li et al. 2012, 2015). The second approach is to start with the achromatic assumption and investigate how bad the data violate such an assumption. By doing so, we reduce one free parameter and impose the same $t_{\mathrm{b}}$ in both bands in the model. We believe that this second approach is more reasonable to address the question of how bad the external forward shock models are, so we adopt the second approach with the help of the first approach. The detailed procedure of our light curve fitting is as follows.

1. For each GRB, we first fit the optical and X-ray afterglow light curves separately and get the respective fitting parameters, such as $t_{\mathrm{O}, \mathrm{b}}, t_{\mathrm{X}, \mathrm{b}}$, and $\omega$ values, of each break. A minimum number of components (SPL, BPL, or TPL) are introduced based on eye inspection of the global features in the light curve. If the reduced $\chi^{2}$ is much larger than one, we continue to add more components and redo the fits, until the reduced $\chi^{2}$ becomes close to one (usually less than 1.5). The reduced $\chi^{2}$ values for some light curves are much smaller than one, indicating that some model parameters are poorly constrained. For these cases, we fix some parameters and redo the fits until the reduced $\chi^{2}$ becomes close to one. Some GRBs have erratic fluctuations in the light curves with small error bars, so the reduced $\chi^{2}$ is much larger than one. For these cases, we do not add additional components to fit the light curves, so their $\chi^{2}$ values remain much larger than one.

2. Next, we jointly fit both the optical and X-ray light curves by introducing the same $t_{\mathrm{b}}$. We search for a possible achromatic break time in the range $\left[t_{\mathrm{O}, \mathrm{b}}, t_{\mathrm{X}, \mathrm{b}}\right]$. We still fit the optical and X-ray light curves at a test break time $t_{\mathrm{b}}$ separately in this step. The individual $\chi^{2}$ of the optical or X-ray band could not represent the goodness of the joint fit. To evaluate the goodness of the fits for optical and $\mathrm{X}$-ray light curves at $t_{\mathrm{b}}$, we introduce a weighted reduced $\chi_{\text {total }}^{2}$, which is essentially the average reduced $\chi^{2}$ in both bands. Take GRB 050922C as an example: a best joint fit is achieved at $t_{\mathrm{b}}=17.3 \mathrm{ks}$, where the reduced $\chi_{\mathrm{X}}^{2}$ values are $175 / 157$ and $175 / 148 \simeq 186 / 157$ for the optical and $\mathrm{X}$-ray bands, respectively, so $\chi_{\text {total }}^{2}$ can be expressed as $361 / 314$. For all of the GRBs, we search for the common $t_{\mathrm{b}}$ with the best $\chi_{\text {total }}^{2}$. We accept the fits with $\chi_{\text {total }}^{2} \leqslant 3$ and regard it as not inconsistent with being achromatic. ${ }^{12}$ Usually the parameters of these best joint fits do not correspond to the best reduced $\chi^{2}$ in each band.

\footnotetext{
12 The adoption of a separation line at $\chi_{\text {total }}^{2} \sim 3$ is somewhat arbitrary, but the value is determined based on close inspection of the fitting results of individual bursts. Our results indicate that most GRB afterglow light curves are well fit with the BPL or SPL light curve models, with a typical value of $\chi_{\text {total }}^{2}=1.21 \pm 0.50$. However, some GRBs (e.g., GRB 050730, 060904B 080319C, 100901A, 120326A) show a relatively large $\chi_{\text {total }}^{2}$, which are around or even slightly larger than three. Inspecting their light curves, the relatively large $\chi_{\text {total }}^{2}$ is caused by complicated features in the light curves (such as small flares and fluctuations), especially in the optical band (e.g., GRB 060904B). However, the PL and BPL fits in any case catch the general features of these light curves. Since we are interested in the achromatic/chromatic properties rather than the flaring features of the light curves, a relatively loose criterion $\left(\chi_{\text {total }}^{2} \sim 3\right)$ is reasonable.
} 
Table 1

Temporal and Spectral Parameters of 85 GRBs

\begin{tabular}{|c|c|c|c|c|c|c|c|c|c|c|c|c|c|}
\hline GRB & $\beta_{\mathrm{O}}$ & $\beta_{\mathrm{X}}$ & $\alpha_{O, 1}{ }^{a}$ & $\alpha_{\mathrm{O}, 2}$ & $\omega$ & Function & $\alpha_{\mathrm{X}, 1}{ }^{\mathrm{a}}$ & $\alpha_{\mathrm{X}, 2}$ & $\omega$ & Function & $\Delta \alpha_{\mathrm{X}, \mathrm{O}^{\mathrm{b}}}$ & $\Delta \beta_{\mathrm{X}, \mathrm{O}}^{\mathrm{c}}$ & $t_{\mathrm{b}}^{\mathrm{d}}$ \\
\hline \multicolumn{14}{|l|}{ Grade I } \\
\hline 050408 & $0.28 \pm 0.33$ & $1.14 \pm 0.14$ & $0.49 \pm 0.01$ & $1.29 \pm 0.11$ & 3 & BPL & $0.73 \pm 0.15$ & $1.17 \pm 0.22$ & 3 & BPL & $-0.12 \pm 0.33$ & $0.86 \pm 0.47$ & 40.7 \\
\hline 050801 & $0.69 \pm 0.34$ & $0.92 \pm 0.17$ & $0.07 \pm 0.01$ & $1.20 \pm 0.01$ & 3 & BPL & $0.24 \pm 0.11$ & $1.18 \pm 0.03$ & 3 & BPL & $-0.02 \pm 0.04$ & $0.23 \pm 0.51$ & 0.2 \\
\hline 050820A & $0.72 \pm 0.03$ & $0.89 \pm 0.05$ & $0.91 \pm 0.02$ & $1.67 \pm 0.09$ & 3 & BPL & $1.12 \pm 0.08$ & $1.89 \pm 0.11$ & 3 & BPL & $0.22 \pm 0.20$ & $0.17 \pm 0.08$ & 2379.0 \\
\hline 050922C & $0.51 \pm 0.05$ & $1.06 \pm 0.11$ & $0.82 \pm 0.11$ & $1.53 \pm 0.09$ & 3 & BPL & $1.04 \pm 0.12$ & $1.71 \pm 0.19$ & 3 & BPL & $0.18 \pm 0.28$ & $0.55 \pm 0.16$ & 8.0 \\
\hline 051028 & $0.60 \pm 0.00$ & $0.95 \pm 0.15$ & $0.99 \pm 0.06$ & $\cdots$ & $\cdots$ & SPL & $1.16 \pm 0.08$ & $\cdots$ & $\cdots$ & SPL & $0.17 \pm 0.14$ & $0.35 \pm 0.15$ & $\cdots$ \\
\hline 051109A & $0.70 \pm 0.05$ & $0.98 \pm 0.08$ & $0.64 \pm 0.08$ & $1.07 \pm 0.12$ & 3 & BPL & $0.24 \pm 0.04$ & $1.22 \pm 0.11$ & 3 & BPL & $0.15 \pm 0.23$ & $0.28 \pm 0.13$ & 3.5 \\
\hline 060111B & $0.70 \pm 0.10$ & $0.95 \pm 0.18$ & $0.80 \pm 0.07$ & $1.55 \pm 0.08$ & 3 & BPL & $0.90 \pm 0.15$ & $1.59 \pm 0.12$ & 3 & BPL & $0.04 \pm 0.20$ & $0.25 \pm 0.28$ & 7.2 \\
\hline 060206 & $0.73 \pm 0.05$ & $1.20 \pm 0.31$ & $0.42 \pm 0.09$ & $1.43 \pm 0.10$ & 3 & BPL & $0.40 \pm 0.09$ & $1.50 \pm 0.06$ & 3 & BPL & $0.07 \pm 0.16$ & $0.47 \pm 0.36$ & 12.5 \\
\hline 060418 & $0.78 \pm 0.09$ & $1.10 \pm 0.10$ & $1.23 \pm 0.07$ & $\cdots$ & 3 & BPL & $1.33 \pm 0.06$ & $\cdots$ & 3 & BPL & $0.10 \pm 0.13$ & $0.32 \pm 0.19$ & $\cdots$ \\
\hline 060512 & $0.68 \pm 0.05$ & $1.04 \pm 0.10$ & $0.81 \pm 0.05$ & $\cdots$ & $\cdots$ & SPL & $1.20 \pm 0.07$ & $\cdots$ & $\cdots$ & SPL & $0.39 \pm 0.12$ & $0.36 \pm 0.15$ & $\cdots$ \\
\hline 060714 & $0.44 \pm 0.04$ & $1.10 \pm 0.19$ & $0.15 \pm 0.06$ & $1.04 \pm 0.15$ & 3 & BPL & $0.48 \pm 0.09$ & $1.34 \pm 0.11$ & 3 & BPL & $0.30 \pm 0.26$ & $0.66 \pm 0.23$ & 5.9 \\
\hline 060729 & $0.78 \pm 0.03$ & $1.02 \pm 0.04$ & $0.10 \pm 0.05$ & $1.40 \pm 0.15$ & 3 & BPL & $0.05 \pm 0.01$ & $1.45 \pm 0.11$ & 3 & BPL & $0.05 \pm 0.26$ & $0.24 \pm 0.07$ & 53.0 \\
\hline 060904B & $1.11 \pm 0.10$ & $1.19 \pm 0.15$ & $1.10 \pm 0.05$ & $\cdots$ & 3 & BPL & $1.41 \pm 0.18$ & $\cdots$ & 3 & BPL & $0.31 \pm 0.23$ & $0.08 \pm 0.25$ & 2.4 \\
\hline 060912A & $0.60 \pm 0.15$ & $0.62 \pm 0.20$ & $0.94 \pm 0.03$ & $\ldots$ & $\ldots$ & SPL & $1.07 \pm 0.02$ & $\cdots$ & $\cdots$ & SPL & $0.13 \pm 0.05$ & $0.02 \pm 0.35$ & $\cdots$ \\
\hline 060927 & $0.61 \pm 0.05$ & $0.77 \pm 0.20$ & $1.30 \pm 0.10$ & $\cdots$ & 3 & BPL & $1.30 \pm 0.07$ & $\cdots$ & 3 & BPL & $0.00 \pm 0.17$ & $0.16 \pm 0.25$ & 0.9 \\
\hline 061007 & $1.02 \pm 0.05$ & $1.00 \pm 0.10$ & $1.62 \pm 0.08$ & $\cdots$ & 3 & BPL & $1.66 \pm 0.07$ & $\cdots$ & $\cdots$ & SPL & $0.04 \pm 0.15$ & $-0.02 \pm 0.15$ & $\cdots$ \\
\hline 061126 & $0.82 \pm 0.09$ & $0.85 \pm 0.17$ & $1.29 \pm 0.04$ & $\cdots$ & 3 & BPL & $1.34 \pm 0.05$ & $\cdots$ & $\cdots$ & SPL & $0.05 \pm 0.09$ & $0.03 \pm 0.26$ & $6.0^{\circ}$ \\
\hline 070318 & $0.78 \pm 0.10$ & $0.97 \pm 0.11$ & $1.02 \pm 0.10$ & $\cdots$ & 3 & BPL & $1.03 \pm 0.02$ & $\cdots$ & $\cdots$ & SPL & $0.01 \pm 0.12$ & $0.19 \pm 0.21$ & $\cdots$ \\
\hline 070411 & 0.75 & $1.24 \pm 0.22$ & $0.50 \pm 0.08$ & $1.50 \pm 0.11$ & 3 & BPL & $1.10 \pm 0.06$ & $1.40 \pm 0.09$ & 3 & BPL & $-0.10 \pm 0.20$ & $0.49 \pm 0.22$ & 65.0 \\
\hline 070518 & 0.80 & $1.20 \pm 0.34$ & $0.70 \pm 0.07$ & $1.80 \pm 0.11$ & 3 & BPL & $0.41 \pm 0.06$ & $1.51 \pm 0.09$ & 3 & BPL & $-0.29 \pm 0.20$ & $0.40 \pm 0.34$ & 40.1 \\
\hline 071025 & $0.96 \pm 0.14$ & $1.08 \pm 0.11$ & $1.43 \pm 0.06$ & $\cdots$ & 3 & BPL & $1.52 \pm 0.08$ & $\cdots$ & $\cdots$ & SPL & $0.09 \pm 0.14$ & $0.12 \pm 0.25$ & $\cdots$ \\
\hline 071031 & $0.64 \pm 0.05$ & $0.71 \pm 0.14$ & $0.79 \pm 0.05$ & $\cdots$ & 3 & BPL & $0.82 \pm 0.05$ & $\cdots$ & $\cdots$ & SPL & $0.03 \pm 0.10$ & $0.07 \pm 0.19$ & $\cdots$ \\
\hline 080319C & $0.98 \pm 0.42$ & $0.61 \pm 0.10$ & $1.12 \pm 0.13$ & $\ldots$ & 3 & BPL & $1.33 \pm 0.08$ & $\cdots$ & $\cdots$ & SPL & $0.21 \pm 0.21$ & $-0.37 \pm 0.52$ & $\cdots$ \\
\hline 080413A & $0.52 \pm 0.37$ & $1.15 \pm 0.24$ & $1.54 \pm 0.05$ & $\cdots$ & 3 & BPL & $1.68 \pm 0.09$ & $\cdots$ & 3 & BPL & $0.14 \pm 0.14$ & $0.63 \pm 0.61$ & 0.3 \\
\hline 080603A & $0.98 \pm 0.04$ & $1.01 \pm 0.10$ & $0.95 \pm 0.03$ & $\cdots$ & 3 & BPL & $0.96 \pm 0.05$ & $\cdots$ & $\cdots$ & SPL & $0.01 \pm 0.08$ & $0.03 \pm 0.14$ & $\cdots$ \\
\hline 080710 & $0.80 \pm 0.09$ & $1.00 \pm 0.11$ & $0.39 \pm 0.05$ & $1.32 \pm 0.11$ & 3 & BPL & $0.34 \pm 0.04$ & $1.57 \pm 0.14$ & 3 & BPL & $0.25 \pm 0.25$ & $0.20 \pm 0.20$ & 6.8 \\
\hline 080804 & 0.43 & $0.82 \pm 0.10$ & $0.87 \pm 0.01$ & $\cdots$ & $\cdots$ & SPL & $1.11 \pm 0.01$ & $\cdots$ & $\cdots$ & SPL & $0.24 \pm 0.02$ & $0.39 \pm 0.10$ & $\cdots$ \\
\hline 080913 & $0.79 \pm 0.03$ & $1.01 \pm 0.23$ & $0.98 \pm 0.02$ & $\cdots$ & $\cdots$ & SPL & $1.32 \pm 0.15$ & $\cdots$ & $\cdots$ & SPL & $0.34 \pm 0.17$ & $0.22 \pm 0.26$ & $\cdots$ \\
\hline 080928 & $1.32 \pm 0.22$ & $1.14 \pm 0.10$ & $2.02 \pm 0.12$ & $\cdots$ & 3 & BPL & $1.81 \pm 0.11$ & $\cdots$ & 3 & BPL & $-0.21 \pm 0.23$ & $-0.18 \pm 0.32$ & 7.1 \\
\hline 081008 & $0.40 \pm 0.23$ & $0.98 \pm 0.11$ & $0.64 \pm 0.06$ & $1.60 \pm 0.09$ & 3 & BPL & $0.87 \pm 0.15$ & $1.68 \pm 0.08$ & 3 & BPL & $0.08 \pm 0.17$ & $0.58 \pm 0.34$ & 9.5 \\
\hline 081203A & 0.60 & $1.04 \pm 0.10$ & $1.15 \pm 0.07$ & $1.87 \pm 0.13$ & 3 & BPL & $1.04 \pm 0.09$ & $1.89 \pm 0.11$ & 3 & BPL & $0.02 \pm 0.24$ & $0.44 \pm 0.10$ & 7.1 \\
\hline 090102 & $0.74 \pm 0.22$ & $0.79 \pm 0.11$ & $0.20 \pm 0.04$ & $1.16 \pm 0.09$ & 3 & BPL & $0.31 \pm 0.05$ & $1.41 \pm 0.09$ & 3 & BPL & $0.25 \pm 0.18$ & $0.05 \pm 0.33$ & 1.0 \\
\hline 090323 & $0.74 \pm 0.15$ & $0.87 \pm 0.22$ & $1.55 \pm 0.05$ & $\cdots$ & $\cdots$ & SPL & $1.62 \pm 0.09$ & $\cdots$ & $\cdots$ & SPL & $0.07 \pm 0.14$ & $0.13 \pm 0.37$ & $\cdots$ \\
\hline 090328 & $1.19 \pm 0.21$ & $0.90 \pm 0.30$ & $1.84 \pm 0.08$ & $\cdots$ & $\cdots$ & SPL & $1.67 \pm 0.11$ & $\cdots$ & $\cdots$ & SPL & $-0.17 \pm 0.19$ & $-0.29 \pm 0.51$ & $\cdots$ \\
\hline 090426 & $0.76 \pm 0.14$ & $1.03 \pm 0.15$ & $0.14 \pm 0.09$ & $1.25 \pm 0.04$ & 3 & BPL & $0.13 \pm 0.02$ & $1.04 \pm 0.05$ & 3 & BPL & $-0.21 \pm 0.09$ & $0.27 \pm 0.29$ & 0.2 \\
\hline 090618 & $0.50 \pm 0.05$ & $0.92 \pm 0.05$ & $0.76 \pm 0.11$ & $1.53 \pm 0.11$ & 3 & BPL & $0.93 \pm 0.09$ & $1.74 \pm 0.10$ & 3 & BPL & $0.21 \pm 0.21$ & $0.42 \pm 0.10$ & 45.1 \\
\hline 090926A & $0.72 \pm 0.17$ & $0.98 \pm 0.15$ & $1.34 \pm 0.05$ & $\cdots$ & $\cdots$ & SPL & $1.41 \pm 0.03$ & $\cdots$ & $\cdots$ & SPL & $0.07 \pm 0.08$ & $0.26 \pm 0.32$ & $\cdots$ \\
\hline 091127 & 0.18 & $0.68 \pm 0.11$ & $0.55 \pm 0.11$ & $1.50 \pm 0.11$ & 3 & BPL & $0.96 \pm 0.05$ & $1.59 \pm 0.12$ & 3 & BPL & $0.09 \pm 0.23$ & $0.50 \pm 0.11$ & 35.3 \\
\hline 100418A & $0.98 \pm 0.09$ & $1.04 \pm 0.29$ & $0.11 \pm 0.01$ & $1.60 \pm 0.10$ & 3 & BPL & $-0.12 \pm 0.03$ & $1.57 \pm 0.11$ & 3 & BPL & $-0.03 \pm 0.21$ & $0.06 \pm 0.38$ & 90.1 \\
\hline $100901 \mathrm{~A}$ & $0.52 \pm 0.10$ & $1.00 \pm 0.30$ & $1.42 \pm 0.02$ & $\cdots$ & 3 & BPL & $1.41 \pm 0.02$ & & 3 & BPL & $-0.01 \pm 0.04$ & $0.48 \pm 0.40$ & 29.8 \\
\hline $101024 \mathrm{~A}$ & $0.70 \pm 0.40$ & $0.82 \pm 0.13$ & $0.01 \pm 0.05$ & $1.07 \pm 0.08$ & 3 & BPL & $-0.09 \pm 0.07$ & $1.37 \pm 0.10$ & 3 & BPL & $0.30 \pm 0.18$ & $0.12 \pm 0.53$ & 1.0 \\
\hline $120326 A$ & $0.75 \pm 0.08$ & $0.77 \pm 0.06$ & $1.52 \pm 0.10$ & $\cdots$ & 3 & BPL & $1.69 \pm 0.09$ & $\cdots$ & 3 & BPL & $0.17 \pm 0.19$ & $0.02 \pm 0.14$ & 35.50 \\
\hline $130427 \mathrm{~A}$ & $0.69 \pm 0.01$ & $0.68 \pm 0.16$ & $1.02 \pm 0.09$ & $1.84 \pm 0.11$ & 3 & BPL & $1.09 \pm 0.07$ & $1.63 \pm 0.09$ & 3 & BPL & $-0.21 \pm 0.20$ & $-0.01 \pm 0.17$ & 127.5 \\
\hline
\end{tabular}


Table 1

(Continued)

\begin{tabular}{|c|c|c|c|c|c|c|c|c|c|c|c|c|c|}
\hline GRB & $\beta_{\mathrm{O}}$ & $\beta_{\mathrm{X}}$ & $\alpha_{\mathrm{O}, 1}{ }^{\mathrm{a}}$ & $\alpha_{\mathrm{O}, 2}$ & $\omega$ & Function & $\alpha_{\mathrm{X}, 1}{ }^{\mathrm{a}}$ & $\alpha_{\mathrm{X}, 2}$ & $\omega$ & Function & $\Delta \alpha_{\mathrm{X}, \mathrm{O}}^{\mathrm{b}}$ & $\Delta \beta_{\mathrm{X}, \mathrm{O}}^{\mathrm{c}}$ & $t_{\mathrm{b}}^{\mathrm{d}}$ \\
\hline 090313 & $0.74 \pm 0.40$ & $1.08 \pm 0.17$ & $1.55 \pm 0.12$ & $\cdots$ & 3 & BPL & $1.67 \pm 0.10$ & $\cdots$ & $\ldots$ & SPL & $0.12 \pm 0.22$ & $0.34 \pm 0.57$ & $20.5^{\circ}$ \\
\hline \multicolumn{14}{|l|}{ Grade III } \\
\hline 050319 & $0.74 \pm 0.42$ & $1.01 \pm 0.07$ & $0.39 \pm 0.06$ & $1.02 \pm 0.04$ & 3 & BPL & $0.58 \pm 0.07$ & $1.72 \pm 0.11$ & 3 & BPL & $0.70 \pm 0.15$ & $0.27 \pm 0.49$ & 55.0 \\
\hline 050401 & $0.50 \pm 0.20$ & $0.79 \pm 0.13$ & $0.50 \pm 0.08$ & $0.89 \pm 0.08$ & 3 & SPL & $0.76 \pm 0.05$ & $1.67 \pm 0.11$ & 3 & BPL & $0.78 \pm 0.19$ & $0.29 \pm 0.33$ & $4.3^{x}$ \\
\hline 050416A & 1.30 & $1.07 \pm 0.11$ & $0.26 \pm 0.07$ & $1.12 \pm 0.12$ & 3 & BPL & $0.66 \pm 0.12$ & $0.99 \pm 0.02$ & 3 & BPL & $-0.13 \pm 0.14$ & $-0.23 \pm 0.11$ & 11.0 \\
\hline $050525 \mathrm{~A}$ & $0.52 \pm 0.08$ & $1.09 \pm 0.16$ & $1.46 \pm 0.07$ & $\ldots$ & 3 & BPL & $1.57 \pm 0.04$ & $\ldots$ & 3 & BPL & $0.11 \pm 0.11$ & $0.57 \pm 0.24$ & $4.2^{\mathrm{O}}$ \\
\hline 050603 & $0.20 \pm 0.10$ & $1.02 \pm 0.13$ & $1.70 \pm 0.13$ & $\ldots$ & $\ldots$ & SPL & $1.71 \pm 0.05$ & $\ldots$ & $\ldots$ & SPL & $0.01 \pm 0.18$ & $0.82 \pm 0.23$ & $\ldots$ \\
\hline 050721 & $1.16 \pm 0.35$ & $0.85 \pm 0.22$ & $0.60 \pm 0.03$ & $\ldots$ & $\ldots$ & SPL & $1.01 \pm 0.08$ & $\ldots$ & $\ldots$ & SPL & $0.41 \pm 0.11$ & $-0.31 \pm 0.57$ & $\ldots$ \\
\hline 050730 & $0.52 \pm 0.05$ & $1.62 \pm 0.04$ & $0.48 \pm 0.05$ & $1.47 \pm 0.06$ & 3 & BPL & $0.45 \pm 0.13$ & $2.64 \pm 0.20$ & 3 & BPL & $1.17 \pm 0.26$ & $1.10 \pm 0.09$ & 90.1 \\
\hline 051221A & $0.64 \pm 0.05$ & $1.06 \pm 0.14$ & $0.34 \pm 0.07$ & $1.24 \pm 0.04$ & 3 & BPL & $0.35 \pm 0.08$ & $1.34 \pm 0.04$ & 3 & BPL & $0.10 \pm 0.08$ & $0.42 \pm 0.19$ & 25.1 \\
\hline 060210 & $0.37 \pm 0.08$ & $1.08 \pm 0.08$ & $0.53 \pm 0.05$ & $1.77 \pm 0.14$ & 3 & BPL & $0.53 \pm 0.06$ & $1.30 \pm 0.12$ & 3 & BPL & $-0.47 \pm 0.26$ & $0.71 \pm 0.16$ & 5.0 \\
\hline 060526 & $0.51 \pm 0.32$ & $0.90 \pm 0.11$ & $0.56 \pm 0.10$ & $1.93 \pm 0.10$ & 1 & BPL & $0.67 \pm 0.08$ & $2.06 \pm 0.15$ & 3 & BPL & $0.13 \pm 0.25$ & $0.39 \pm 0.43$ & 50.1 \\
\hline 060605 & 1.06 & $1.02 \pm 0.09$ & $0.13 \pm 0.09$ & $2.64 \pm 0.15$ & 3 & BPL & $0.55 \pm 0.08$ & $2.82 \pm 0.15$ & 3 & BPL & $0.18 \pm 0.30$ & $-0.04 \pm 0.09$ & 15.0 \\
\hline 060614 & $0.47 \pm 0.04$ & $0.90 \pm 0.09$ & $-0.35 \pm 0.04$ & $1.90 \pm 0.12$ & 3 & BPL & $0.11 \pm 0.10$ & $1.97 \pm 0.15$ & 3 & BPL & $0.07 \pm 0.27$ & $0.43 \pm 0.13$ & 44.0 \\
\hline 060906 & $0.56 \pm 0.02$ & $1.08 \pm 0.17$ & $1.45 \pm 0.21$ & $\ldots$ & 3 & BPL & $1.25 \pm 0.11$ & $\ldots$ & 3 & BPL & $-0.20 \pm 0.32$ & $0.52 \pm 0.19$ & 1.3 \\
\hline 060906 & $0.56 \pm 0.02$ & $1.08 \pm 0.17$ & $1.44 \pm 0.20$ & $\ldots$ & 3 & BPL & $1.90 \pm 0.11$ & $\ldots$ & 3 & BPL & $0.46 \pm 0.31$ & $0.52 \pm 0.19$ & 10.5 \\
\hline 060908 & $0.24 \pm 0.20$ & $1.13 \pm 0.19$ & $0.71 \pm 0.04$ & $1.11 \pm 0.09$ & 3 & BPL & $0.54 \pm 0.05$ & $1.66 \pm 0.05$ & 3 & BPL & $0.55 \pm 0.14$ & $0.89 \pm 0.39$ & 1.1 \\
\hline 070110 & $0.55 \pm 0.04$ & $1.08 \pm 0.11$ & $0.16 \pm 0.06$ & $1.67 \pm 0.12$ & 3 & BPL & $0.30 \pm 0.10$ & $5.10 \pm 0.30$ & 3 & BPL & $3.43 \pm 0.42$ & $0.53 \pm 0.15$ & 20.3 \\
\hline 070125 & $0.59 \pm 0.10$ & $1.03 \pm 0.20$ & $2.96 \pm 0.05$ & $\ldots$ & $\ldots$ & BPL & $2.12 \pm 0.04$ & $\ldots$ & 3 & BPL & $-0.84 \pm 0.09$ & $0.44 \pm 0.30$ & 101.1 \\
\hline 070306 & $0.70 \pm 0.10$ & $0.95 \pm 0.09$ & $1.22 \pm 0.11$ & $\ldots$ & 3 & BPL & $2.03 \pm 0.04$ & $\ldots$ & 3 & BPL & $0.81 \pm 0.15$ & $0.25 \pm 0.19$ & 36.9 \\
\hline 070311 & $1.00 \pm 0.20$ & $1.00 \pm 0.24$ & $0.73 \pm 0.02$ & $\ldots$ & $\ldots$ & SPL & $1.09 \pm 0.06$ & $\ldots$ & $\ldots$ & SPL & $\ldots$ & $0.00 \pm 0.44$ & $\ldots$ \\
\hline 070419A & $0.48 \pm 0.48$ & $1.20 \pm 0.30$ & $1.28 \pm 0.04$ & $\ldots$ & 3 & BPL & $0.60 \pm 0.02$ & $\ldots$ & 3 & BPL & $-0.68 \pm 0.06$ & $0.72 \pm 0.78$ & $\ldots$ \\
\hline 071010A & $0.61 \pm 0.12$ & $1.30 \pm 0.40$ & $2.19 \pm 0.08$ & $\ldots$ & 3 & BPL & $1.89 \pm 0.07$ & $\ldots$ & 3 & BPL & $-0.30 \pm 0.15$ & $0.69 \pm 0.52$ & 70.1 \\
\hline 071112C & $0.63 \pm 0.29$ & $0.67 \pm 0.13$ & $0.30 \pm 0.07$ & $0.95 \pm 0.11$ & 3 & BPL & $0.50 \pm 0.08$ & $1.49 \pm 0.11$ & 3 & BPL & $0.54 \pm 0.22$ & $0.04 \pm 0.42$ & 1.5 \\
\hline 080310 & $0.42 \pm 0.12$ & $0.95 \pm 0.18$ & $0.11 \pm 0.01$ & $1.24 \pm 0.02$ & 3 & BPL & $0.03 \pm 0.06$ & $1.24 \pm 0.08$ & 3 & BPL & $0.00 \pm 0.10$ & $0.53 \pm 0.30$ & 5.1 \\
\hline 080319A & $0.77 \pm 0.02$ & $0.89 \pm 0.10$ & $0.65 \pm 0.07$ & $\ldots$ & 3 & BPL & $0.94 \pm 0.05$ & $\ldots$ & $\ldots$ & $\ldots$ & $0.29 \pm 0.12$ & $0.12 \pm 0.12$ & $\ldots$ \\
\hline 080319B & $0.51 \pm 0.26$ & $0.81 \pm 0.07$ & $0.93 \pm 0.11$ & $1.60 \pm 0.12$ & 3 & BPL & $0.73 \pm 0.05$ & $2.73 \pm 0.16$ & 3 & BPL & $1.13 \pm 0.28$ & $0.30 \pm 0.33$ & 3.0 \\
\hline 080319B & $0.51 \pm 0.26$ & $0.81 \pm 0.07$ & $1.03 \pm 0.10$ & $1.69 \pm 0.09$ & 3 & BPL & $1.43 \pm 0.09$ & $2.19 \pm 0.11$ & 3 & BPL & $0.50 \pm 0.20$ & $0.30 \pm 0.33$ & 690.7 \\
\hline 080413B & $0.25 \pm 0.07$ & $0.94 \pm 0.07$ & $0.31 \pm 0.15$ & $1.89 \pm 0.22$ & 3 & BPL & $0.92 \pm 0.16$ & $1.91 \pm 0.23$ & 3 & BPL & $0.02 \pm 0.45$ & $0.69 \pm 0.14$ & 148.5 \\
\hline 080721 & $0.68 \pm 0.02$ & $0.94 \pm 0.06$ & $1.17 \pm 0.03$ & $1.31 \pm 0.05$ & 3 & BPL & $0.81 \pm 0.01$ & $1.65 \pm 0.07$ & 3 & BPL & $0.34 \pm 0.12$ & $0.26 \pm 0.08$ & 3.1 \\
\hline 090510 & $0.85 \pm 0.05$ & $0.75 \pm 0.12$ & $0.84 \pm 0.05$ & $\ldots$ & 3 & BPL & $2.27 \pm 0.06$ & $\ldots$ & 3 & BPL & $1.43 \pm 0.11$ & $-0.10 \pm 0.17$ & 1.5 \\
\hline 090812 & 0.36 & $0.89 \pm 0.14$ & $1.27 \pm 0.05$ & $\ldots$ & 3 & BPL & $1.22 \pm 0.09$ & $\ldots$ & $\ldots$ & SPL & $-0.05 \pm 0.14$ & $0.53 \pm 0.14$ & $\ldots$ \\
\hline 091029 & $0.49 \pm 0.12$ & $1.12 \pm 0.08$ & $0.48 \pm 0.06$ & $1.34 \pm 0.09$ & 3 & BPL & $0.32 \pm 0.09$ & $1.35 \pm 0.08$ & 3 & BPL & $0.01 \pm 0.17$ & $0.63 \pm 0.20$ & 20.8 \\
\hline $100219 \mathrm{~A}$ & 0.56 & $0.69 \pm 0.23$ & $0.74 \pm 0.08$ & $1.91 \pm 0.12$ & 3 & BPL & $0.54 \pm 0.07$ & $1.65 \pm 0.15$ & 3 & BPL & $-0.26 \pm 0.27$ & $0.13 \pm 0.23$ & 1.8 \\
\hline $100219 \mathrm{~A}$ & 0.56 & $0.69 \pm 0.23$ & $2.21 \pm 0.13$ & $\ldots$ & 3 & BPL & $2.51 \pm 0.16$ & $\ldots$ & 3 & BPL & $0.30 \pm 0.29$ & $0.13 \pm 0.23$ & 20.5 \\
\hline $110205 \mathrm{~A}$ & $0.49 \pm 0.08$ & $0.78 \pm 0.06$ & $1.51 \pm 0.08$ & $\ldots$ & 3 & SPL & $1.59 \pm 0.02$ & $\ldots$ & $\ldots$ & SPL & $0.08 \pm 0.10$ & $0.29 \pm 0.14$ & $\ldots$ \\
\hline $110918 \mathrm{~A}$ & $0.42 \pm 0.18$ & $0.89 \pm 0.30$ & $1.65 \pm 0.07$ & $\ldots$ & $\ldots$ & SPL & $1.61 \pm 0.12$ & $\ldots$ & $\ldots$ & SPL & $-0.04 \pm 0.19$ & $0.47 \pm 0.48$ & $\ldots$ \\
\hline $120729 \mathrm{~A}$ & $1.00 \pm 0.10$ & $0.80 \pm 0.17$ & $0.94 \pm 0.05$ & $2.27 \pm 0.09$ & 3 & BPL & $1.09 \pm 0.06$ & $2.40 \pm 0.16$ & 3 & BPL & $0.13 \pm 0.25$ & $-0.20 \pm 0.27$ & 6.61 \\
\hline $120815 \mathrm{~A}$ & $0.78 \pm 0.01$ & $0.72 \pm 0.11$ & $0.63 \pm 0.04$ & $\cdots$ & 3 & BPL & $0.86 \pm 0.06$ & $\cdots$ & $\cdots$ & SPL & $0.23 \pm 0.10$ & $-0.06 \pm 0.12$ & $\cdots$ \\
\hline \multicolumn{14}{|l|}{ Grade IV } \\
\hline 070611 & $0.73 \pm 0.00$ & $0.83 \pm 0.31$ & $0.58 \pm 0.04$ & $\cdots$ & 3 & BPL & $1.34 \pm 0.12$ & $\cdots$ & 3 & BPL & $0.76 \pm 0.16$ & $0.10 \pm 0.31$ & $33.7^{x}$ \\
\hline 071003 & $0.35 \pm 0.11$ & $0.91 \pm 0.12$ & $1.62 \pm 0.11$ & $\ldots$ & 3 & BPL & $1.63 \pm 0.02$ & $\ldots$ & $\ldots$ & SPL & $0.01 \pm 0.13$ & $0.56 \pm 0.23$ & $\ldots$ \\
\hline $120711 \mathrm{~A}$ & $0.52 \pm 0.02$ & $0.81 \pm 0.10$ & $0.96 \pm 0.04$ & $\ldots$ & 3 & BPL & $1.64 \pm 0.05$ & $\ldots$ & $\ldots$ & SPL & $0.68 \pm 0.09$ & $0.29 \pm 0.12$ & $\ldots$ \\
\hline
\end{tabular}


Table 1

(Continued)

\begin{tabular}{|c|c|c|c|c|c|c|c|c|c|c|c|c|c|}
\hline GRB & $\beta_{\mathrm{O}}$ & $\beta_{\mathrm{X}}$ & $\alpha_{\mathrm{O}, 1}{ }^{\mathrm{a}}$ & $\alpha_{\mathrm{O}, 2}$ & $\omega$ & Function & $\alpha_{\mathrm{X}, 1}{ }^{\mathrm{a}}$ & $\alpha_{\mathrm{X}, 2}$ & $\omega$ & Function & $\Delta \alpha_{\mathrm{X}, \mathrm{O}}{ }^{\mathrm{b}}$ & $\Delta \beta_{\mathrm{X}, \mathrm{O}}{ }^{\mathrm{c}}$ & $t_{\mathrm{b}}^{\mathrm{d}}$ \\
\hline $060607 \mathrm{~A}$ & $0.72 \pm 0.27$ & $0.62 \pm 0.06$ & -0.93 & 4.60 & 3 & BPL & $0.36 \pm 0.03$ & $3.10 \pm 0.12$ & 3 & BPL & $\ldots$ & $-0.10 \pm 0.33$ & $9.5^{x}$ \\
\hline 070208 & 0.68 & $1.20 \pm 0.20$ & $0.49 \pm 0.06$ & $\cdots$ & 3 & BPL & $0.43 \pm 0.03$ & $1.78 \pm 0.13$ & 3 & BPL & $\cdots$ & $0.52 \pm 0.20$ & $9.0^{x}$ \\
\hline 070420 & $\ldots$ & $\ldots$ & $-1.43 \pm 0.04$ & $0.90 \pm 0.08$ & 3 & BPL & $0.12 \pm 0.01$ & $1.46 \pm 0.05$ & 3 & BPL & $\ldots$ & $\ldots$ & $3.0^{x}$ \\
\hline
\end{tabular}

${ }^{a}$ For single power-law (SPL) decay light curves (as described in Section 3), the decay indices are also denoted as $\alpha_{1}$.

${ }^{\mathrm{b}} \Delta \alpha_{\mathrm{X}, \mathrm{O}}=\alpha_{\mathrm{X}, 2}-\alpha_{\mathrm{O}, 2}$

c $\Delta \beta_{\mathrm{X}, \mathrm{O}}=\beta_{\mathrm{X}}-\beta_{\mathrm{O}}$

d In units of ks. The symbols "X" and "O" denote the X-ray and optical bands, respectively. 
3. If both the optical and X-ray light curves decay as an SPL, we do not need to search for a common break time. The weighted reduced $\chi_{\text {total }}^{2}$ is calculated based on the above algorithm for the SPL fits in each band.

4. If one band decays as a BPL, while the other band does not have enough data to search for a break time and decays as an SPL (e.g., the Grade II or IV in Section 3), we impose the $t_{\mathrm{b}}$ identified in the first band as the common $t_{\mathrm{b}}$ and perform the $\chi_{\text {total }}^{2}$ analysis as described above.

The fitted results are presented in Figures 1-5. The parameters of the PL or BPL fits of all the light curves are presented in Table 1. Some light curves have additional features (e.g., steep decay phase, flares, rebrightening features) in one band. We do not report them in Table 1. Our analysis below discards these extra components because they likely arise from additional emission components (e.g., in the internal dissipation regions such as internal shocks and internal magnetic dissipation sites) other than the external shock.

\section{EXTERNAL SHOCK MODELS: CLOSURE RELATIONS AND LIGHT CURVE TYPES}

\subsection{Closure Relations}

The standard external shock models of GRB afterglows have clear theoretical predictions that can be verified or falsified by the observational data. These models attribute the multiwavelength afterglow emission to synchrotron radiation of electrons accelerated in the shock front as the fireball jet interacts with the circumburst medium. The models largely do not depend on the details of the central engine activities, so the afterglow behaviors only depend on a limited number of parameters. In the convention of $F_{\nu} \propto t^{-\alpha} \nu^{-\beta}$, where $\alpha$ and $\beta$ are the temporal and spectral indices of the afterglows that can be measured directly from observations, the models predict certain relationships between the $\alpha$ and $\beta$ values, which are called the "closure relations" of the models (e.g., Zhang \& Mészáros 2004; Zhang et al. 2006; Gao et al. 2013). Technically there are many submodels (e.g., interstellar medium (ISM) versus wind, adiabatic versus radiative, whether or not there is energy injection), physical regimes (reverse shock crossing phase, self-similar phase, post-jet-break phase, Newtonian phase), and spectral regimes (different orders among the observed frequency $(\nu)$ and several characteristic frequencies $\left(\nu_{\mathrm{m}}, \nu_{\mathrm{c}}\right.$, the self-absorption frequency $\left.\left.\nu_{\mathrm{a}}\right)\right)$. We refer to a comprehensive review of Gao et al. (2013) and references therein for the details of various models.

For the time frame of our interest (hours to weeks after the trigger), the reverse shock crossing phase is usually over, and the blast wave is still in the relativistic phase. This greatly reduces the number of relevant models. In Table 2, we summarize the $\alpha$ and $\beta$ predictions of various models studied in this paper following Zhang et al. (2006) and Gao et al. (2013). This includes the ISM and wind models for adiabatic blast waves, ${ }^{13}$ for both pre- and post-jet-break temporal phases, with

\footnotetext{
${ }^{13}$ In general, the circumburst medium can be described by an arbitrary profile $n \propto r^{-k}$. The ISM model corresponds to $k=0$, and the wind model corresponds to $k=2$. In our closure relations, we only consider these two cases because they are naturally expected from the ISM and a preexplosion stellar wind. For other $k$ values, it is not straightforward to imagine a physical mechanism to produce such profiles over a large distance scale of interest. We therefore do not include the arbitrary $k$ models in the standard afterglow models, but discuss them as possible modified afterglow models.
}

and without continuous energy injection, and for two spectral regimes (I: $\nu>\nu_{\mathrm{c}}$ and II: $\nu_{\mathrm{m}}<\nu<\nu_{\mathrm{c}}$ ) in the slow cooling $\left(\nu_{\mathrm{m}}<\nu_{\mathrm{c}}\right)$ regime. By doing so, we have assumed that $\nu_{\mathrm{a}}<\min \left(\nu_{\mathrm{m}}, \nu_{\mathrm{c}}\right)$ and $\min \left(\nu_{\mathrm{X}}, \nu_{\mathrm{O}}\right)>\nu_{\mathrm{m}}$, which is usually satisfied for optical and X-ray afterglow emission for typical GRB parameters.

The energy injection model invokes either a long-lasting central engine (Dai \& Lu 1998; Zhang \& Mészáros 2001) or Lorentz-factor-stratified ejecta (Rees \& Mészáros 1998; Sari \& Mészáros 2000; Uhm et al. 2012). The two scenarios are equivalent with each other in terms of light curve behaviors given a relationship between the central engine parameter $q$ and the stratification parameter $k$ (Zhang et al. 2006). We adopt the description of a long-lasting central engine with a power-law luminosity history $L(t)=L_{0}\left(\frac{t}{t_{0}}\right)^{-q}$ (Zhang \& Mészáros 2001), so the injected energy is $E_{\text {inj }}=\frac{L_{0} t_{0}^{q}}{1-q} t^{1-q}$. The prescription applies when $q<1$. The relevant closure relations are presented in Table 2.

Many observations suggest that GRB outflows are collimated. Assuming a conical jet with opening angle $\theta_{j}$, a steepening in the afterglow light curve is predicted when $1 / \Gamma>\theta_{j}$ ( $\Gamma$ is the bulk Lorentz factor of the blast wave). The main reason for this steepening is the so-called "edge effect" (e.g., Panaitescu et al. 1998): ${ }^{14}$ the $1 / \Gamma$ cone is no longer filled with emission beyond the jet break time (when $1 / \Gamma>\theta_{j}$ ). There is a reduction factor in flux $\theta_{j}^{2} /(1 / \Gamma)^{2}=\Gamma^{2} \theta_{j}^{2}$. The relevant closure relations are also presented in Table 2 .

It is possible that in some GRBs the energy injection phase lasts longer than the jet break time, so a jet break with energy injection in both the pre- and postbreak phases can be observed. The relevant closure relations of such models were derived in Gao et al. (2013) and are also presented in Table 2.

\subsection{Type of Afterglow Light Curves}

For the time domain we are interested in and for the optical and X-ray bands, there are four types of light curves (Figure 6).

(1) BPL light curves with an energy injection break: in reference to the canonical X-ray light curve (Zhang et al. 2006), as reproduced in Figure 6(a), the energy injection break connects the shallow decay phase (segment II) to the normal decay phase (segment III), and a typical light curve is shown in Figure 6(b). Before and after the break, the adiabatic deceleration $\alpha(\beta)$ relations with and without energy injection (as listed in Table 2, Zhang et al. 2006; Gao et al. 2013) are used to check whether the data are consistent with model predictions.

(2) BPL light curves with a jet break: this corresponds to the transition from segment III to IV in the canonical light curve, and a typical light curve is shown in Figure 6(c), upper curve. Light curves in such a category should satisfy the constantenergy, isotropic closure relations before the break and the edge-effect post-jet-break closure relations after the break, with no energy injection effect either before or after the break (Table 2). The postbreak decay index is required to be steeper than 1.5 for this model.

\footnotetext{
${ }^{14}$ Sideways expansion has been discussed as another factor in steepening the light curves (Rhoads 1999; Sari et al. 1999). However, later numerical simulations suggest that this effect is not important (e.g., Zhang \& MacFadyen 2009). We do not consider this effect in this paper.
} 

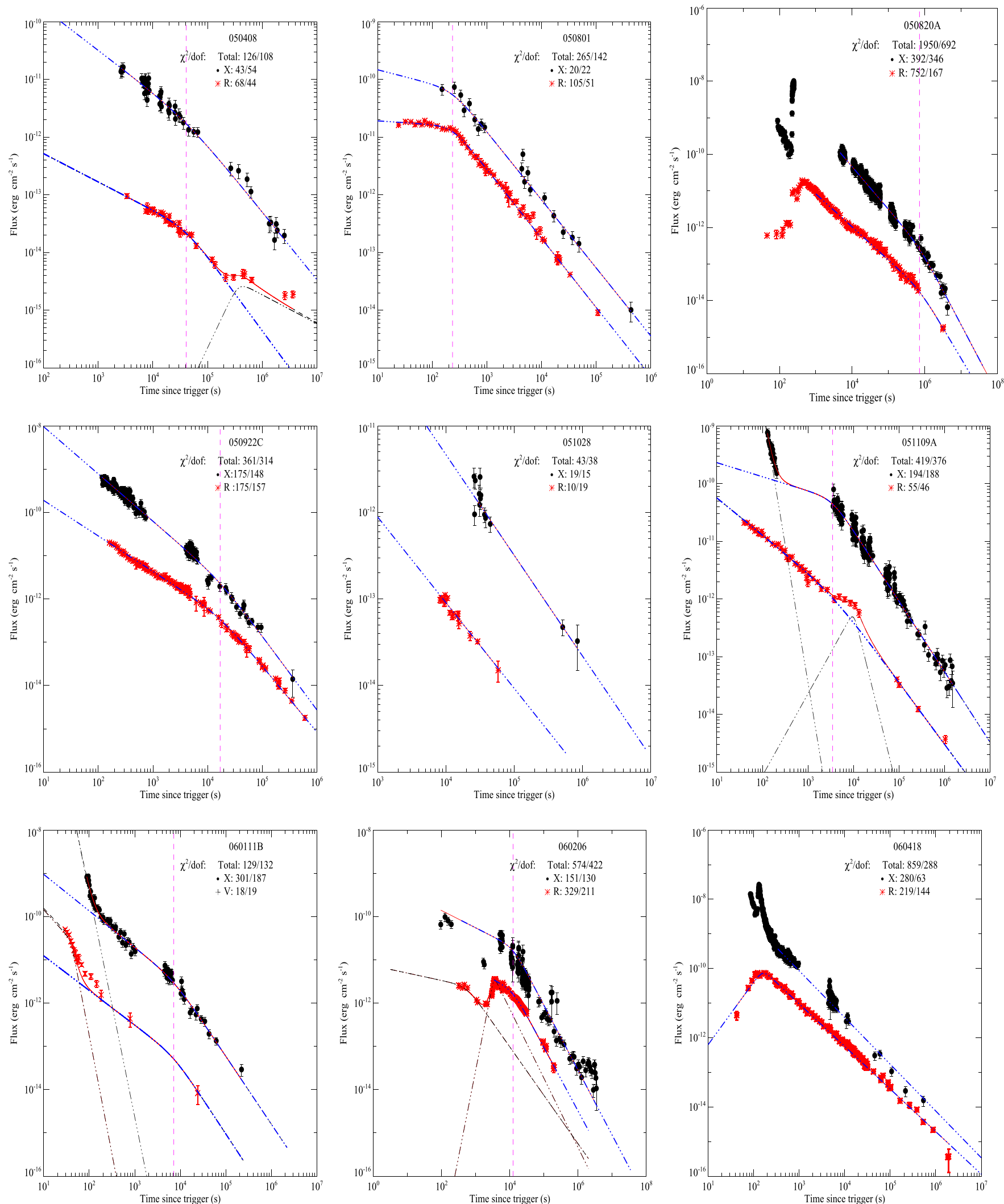

Figure 1. X-ray and optical light curves, as well as the fitting results (blue dot-dashed lines) for the GRBs in the Grade I sample. If an achromatic break exists, the achromatic break time is shown by a purple, vertical dashed line.

(3) BPL light curves with a jet break with energy injection: This model allows the energy injection to extend longer than the jet break. The temporal break is still defined by the edge effect of a canonical jet, but the decay slopes before and after the break are shallower than the previous case (lower curve in Figure 6(c)), so a $q$ parameter is introduced for both the pre- and postbreak phases. 

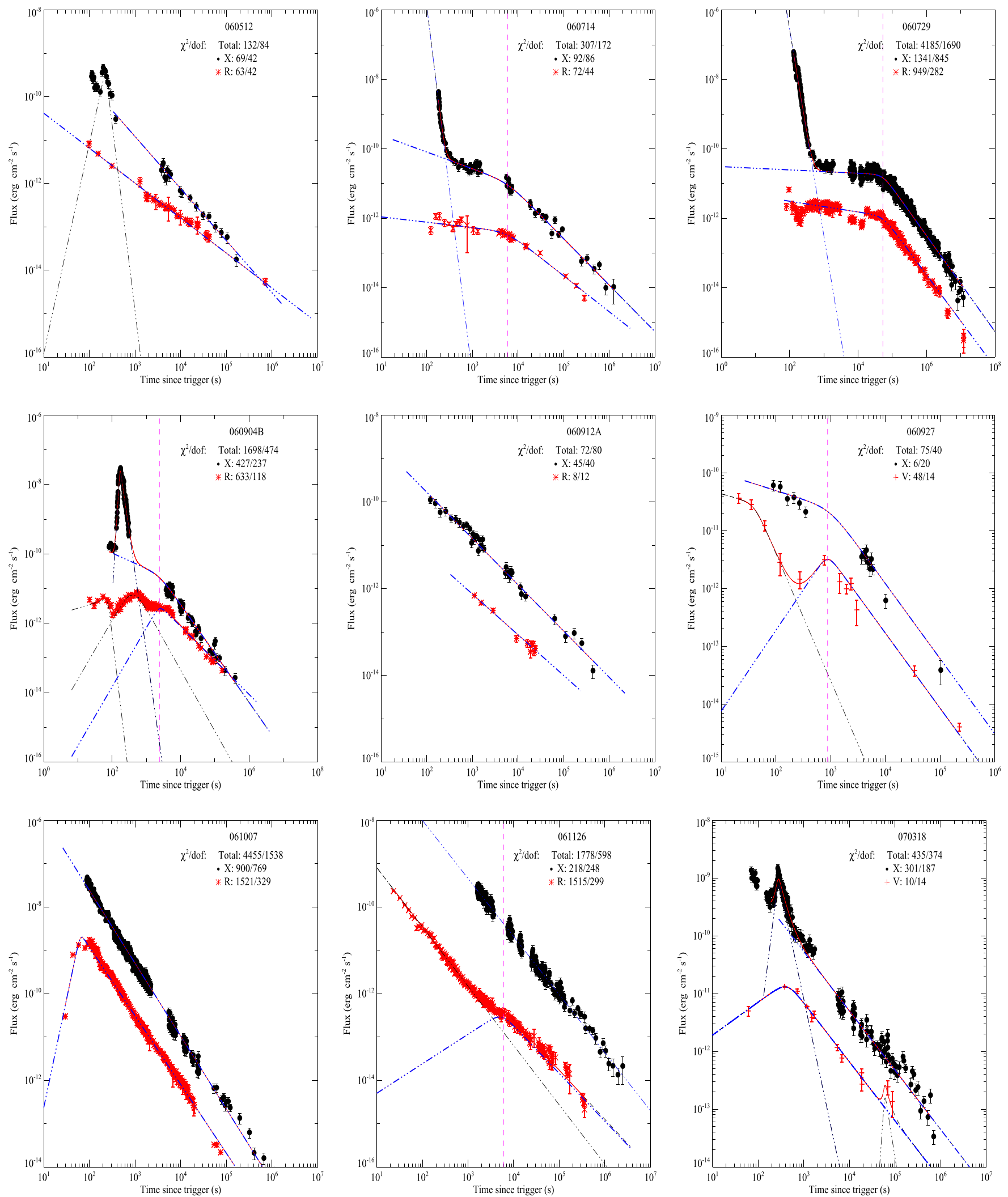

Figure 1. (Continued.)

(4) SPL decay: for some GRBs, a SPL function is adequate to describe the afterglow data (Figure 6(d)) after the deceleration phase. In the X-ray band, there might be a steeper decay phase before this SPL phase, which is due to the tail emission from the prompt emission (Barthelmy et al. 2005; Tagliaferri et al. 2005; Zhang et al. 2006). We ignore the steep decay phase and treat it as an SPL decay (upper curve of Figure 6(d)). Similarly, in the optical band, some GRBs show 

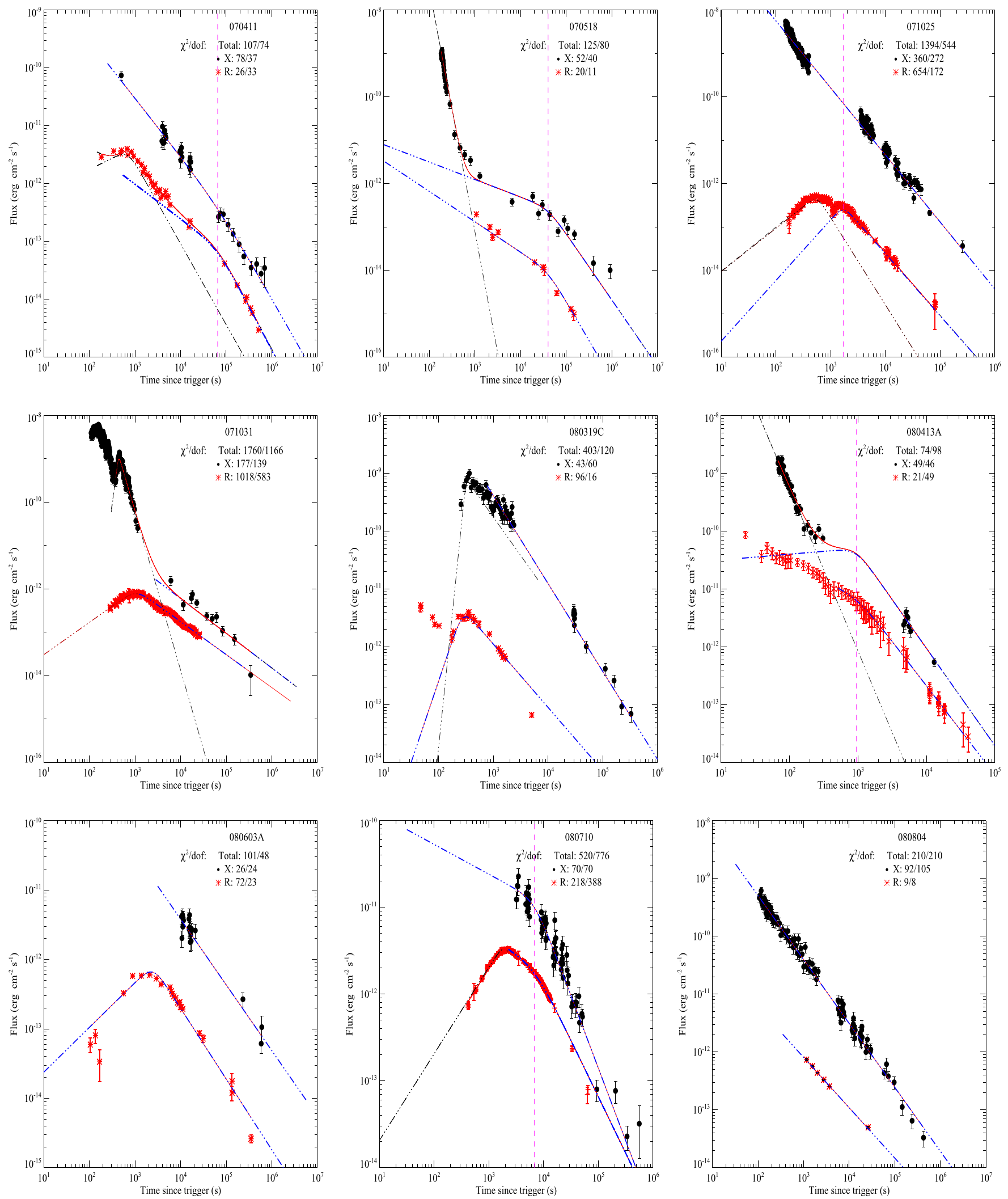

Figure 1. (Continued.)

an early rising phase, which is a signature of the onset of afterglow at the deceleration radius (peak of the light curve, lower curve of Figure 6(d)). We also treat these light curves as SPL decay ones.
For all of the types, sometimes there are X-ray flares overlapping the power-law decay segments. We do not include the flares in our data fitting because they originate from a different emission component due to late central 

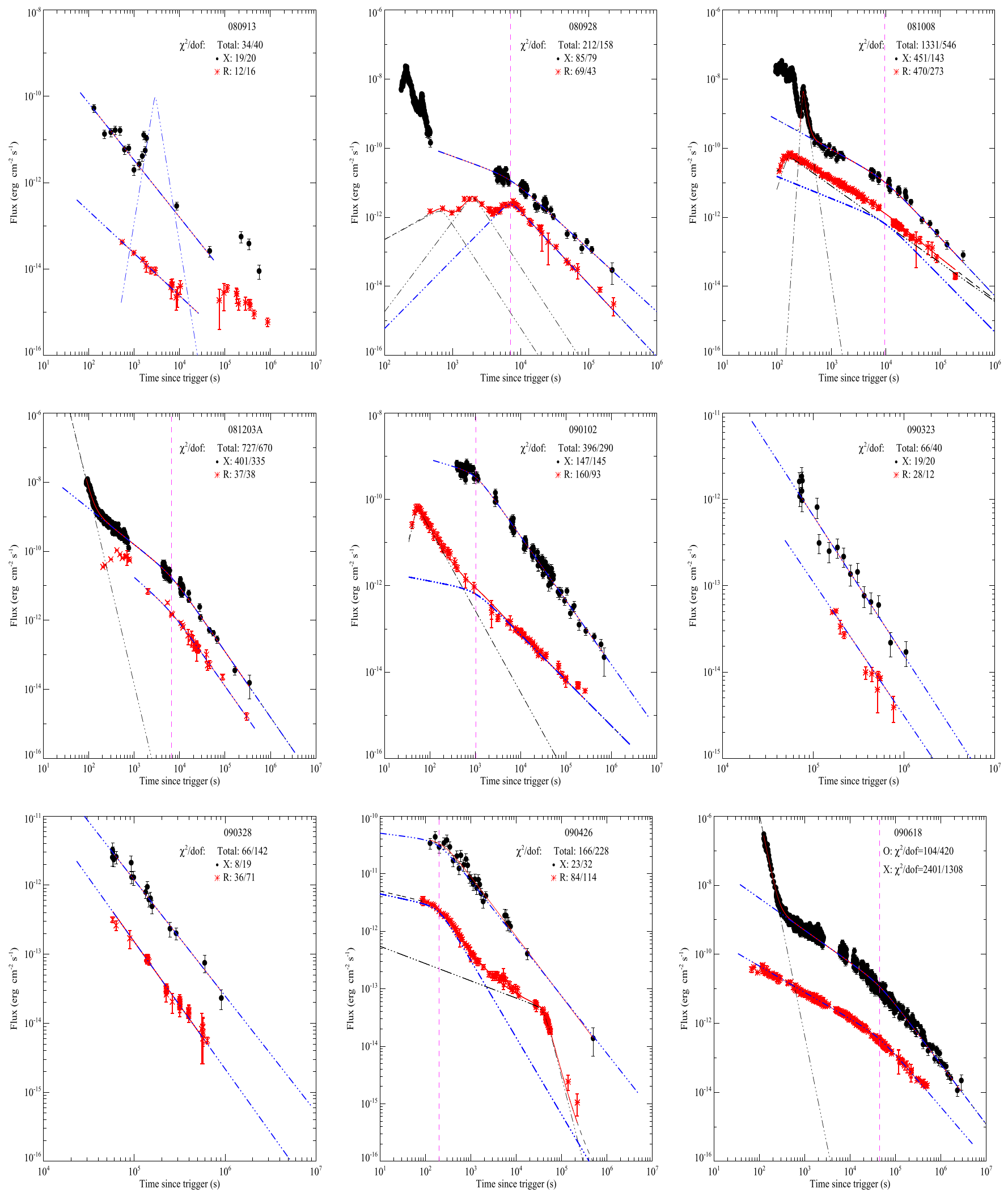

Figure 1. (Continued.)

engine activities (e.g., Zhang et al. 2006; Maxham \& Zhang 2009).

One important task is to perform a self-consistency check between the optical and X-ray bands. If a GRB is consistent with the external forward shock model, we demand that the GRB satisfy the following criteria.

1. The X-ray and optical light curves are consistent with having an achromatic break, if any. 

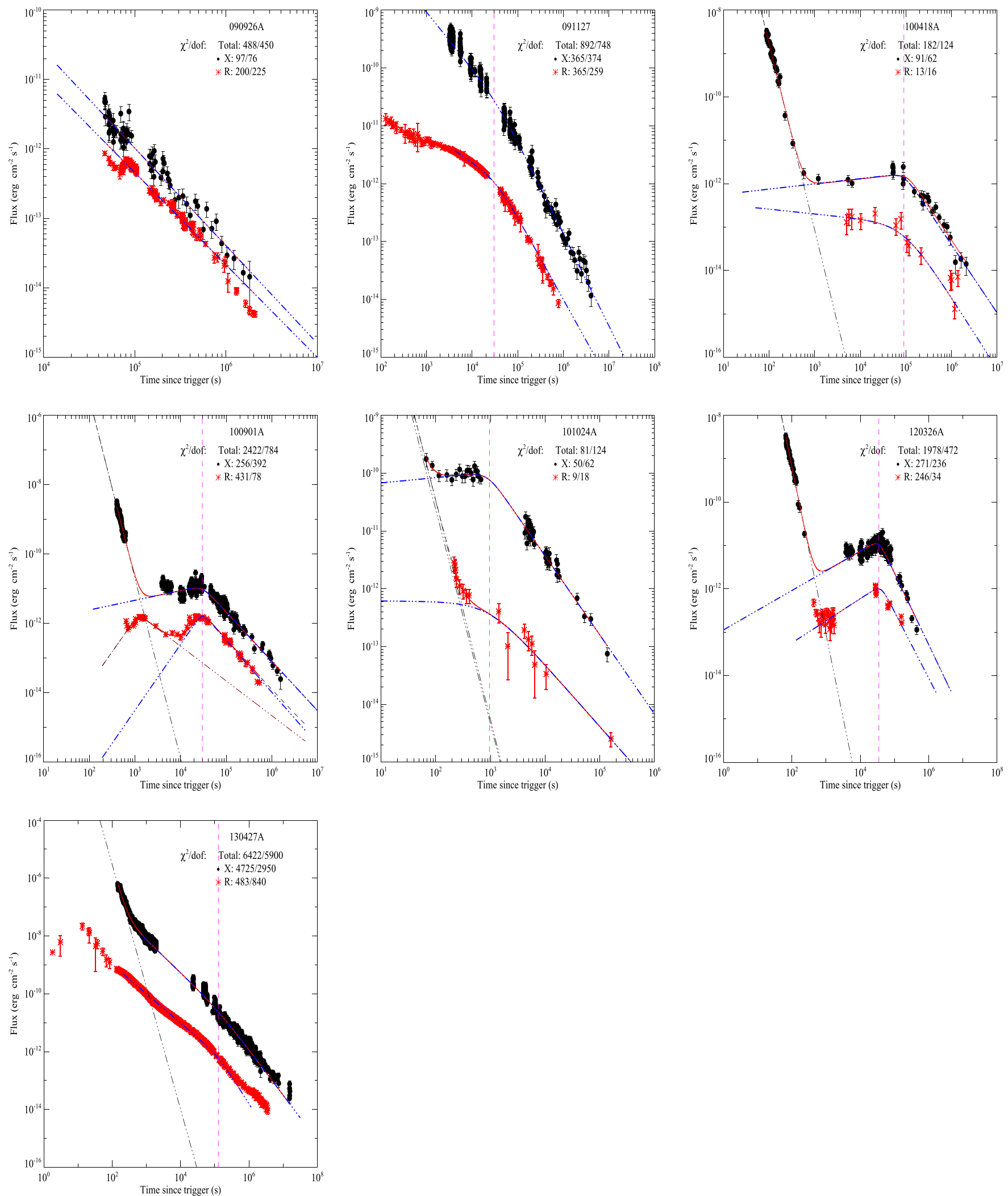

Figure 1. (Continued.)

2. Both the X-ray and optical light curves should satisfy the closure relations of the same circumburst medium type (ISM or wind) in both the pre- and postbreak temporal segments.
3. Either both bands belong to the same spectral regime, or the two bands are separated by a cooling break $\nu_{\mathrm{c}}$, with the X-ray band above the break and the optical band 

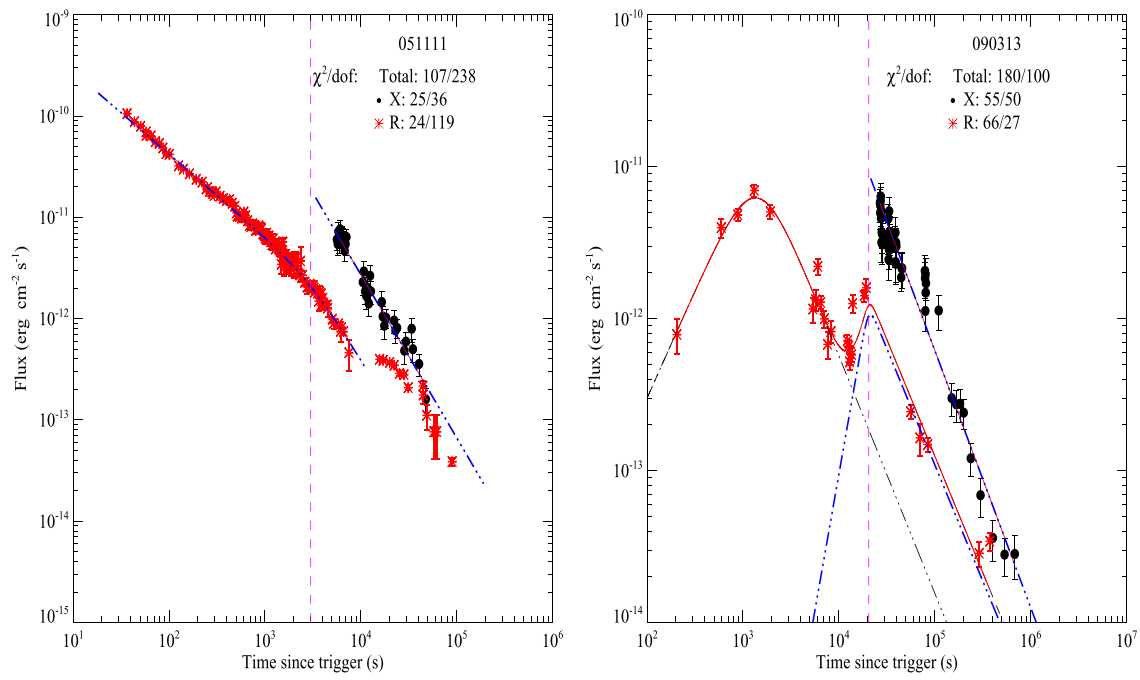

Figure 2. Same as Figure 1, but for the Grade II sample.

below the break (with allowance for a gray zone; see more discussion below).

4. The inferred electron spectral index $p$ from both bands and from both pre- and postbreak segments should be consistent with each other within errors.

5. For energy injection models, the energy injection parameter $q$ values derived from the X-ray and optical bands should be consistent with each other.

Technically, we check the consistency between the closure relations for an individual temporal segment in an individual energy band. To ensure the same $p$ value is derived for different bands, we also check the consistency between the data and models in the $\Delta \beta_{\mathrm{X}, \mathrm{O}}-\Delta \alpha_{\mathrm{X}, \mathrm{O}}$ plane. Here $\Delta \alpha_{\mathrm{X}, \mathrm{O}}=\alpha_{\mathrm{X}}-\alpha_{\mathrm{O}}$ is the difference between the decay indices in the X-ray and optical bands, respectively, in the same temporal segment, and $\Delta \beta_{\mathrm{X}, \mathrm{O}}=\beta_{\mathrm{X}}-\beta_{\mathrm{O}}$ is the difference between the spectral indices in the $\mathrm{X}$-ray and optical bands, respectively. Based on the closure relations (Table 2), one can derive the $\Delta \beta_{\mathrm{X}, \mathrm{O}}-\Delta \alpha_{\mathrm{X}, \mathrm{O}}$ relations of all of the models (Tables 3 and 4). One can see that even though the $\alpha$ and $\beta$ values can be very different in different models, the $\Delta \alpha_{\mathrm{X}, \mathrm{O}}$ and $\Delta \beta_{\mathrm{X}, \mathrm{O}}$ values have several well-predicted values. In particular, for the SPL and jet break models, both pre- and postbreak values are well-defined constants. For the energy injection breaks, the postbreak segment does not depend on the free parameter $q$. As a result, if one focuses on the second component only, all of the models can be expressed as several representative coordinate values in the $\Delta \beta_{\mathrm{X}, \mathrm{O}}-\Delta \alpha_{\mathrm{X}, \mathrm{O}}$ plane. Considering the possible gray zones (see below for details), these points define several straight lines in the $\Delta \beta_{\mathrm{X}, \mathrm{O}}-\Delta \alpha_{\mathrm{X}, \mathrm{O}}$ space (Figure 7 for details). If the observed data intersect with these model lines (within errors), one can regard them as being consistent with the model predictions.

Taking the energy injection break as an example, our analysis uses the following procedure. (1) Use the observed spectral indices $\beta_{\mathrm{O}}$ and $\beta_{\mathrm{X}}$ to predict the postbreak temporal indices $\alpha_{\mathrm{O}, 2}$ and $\alpha_{\mathrm{X}, 2}$ in two possible spectral regimes. Then compare these theoretical predictions with the observational values. If the theoretical values are consistent with the fitting results within errors, then go to the next step. Otherwise, it indicates that this GRB does not fall into this light curve type.
(2) Use the identified spectral regime to calculate the electron spectral index $p$ from the spectral index $\beta$, i.e., $p=2 \beta+1$ for $\nu_{\mathrm{m}}<\nu<\nu_{\mathrm{c}}$, or $p=2 \beta$ for $\nu>\nu_{\mathrm{c}}$. Compare the $p$ values derived from the optical and $\mathrm{X}$-ray data, respectively. If $p_{\mathrm{O}}=p_{\mathrm{X}}$ within errors, then move to the next step. Otherwise, this GRB does not fall into such a light curve type. (3) Use the inferred $p$ value and spectral regimes to infer the energy injection parameter $q$ using the temporal index before the break $\left(\alpha_{\mathrm{O}, 1}\right.$ and $\left.\alpha_{\mathrm{X}, 1}\right)$. Compare the derived $q$ values from the optical and X-ray bands, respectively. If $q_{\mathrm{O}}=q_{\mathrm{X}}$ within errors, then move to the next step. Otherwise, this GRB does not fall into such a light curve type. (4) Using the $\Delta \beta_{\mathrm{X}, \mathrm{O}}-\Delta \alpha_{\mathrm{X}, \mathrm{O}}$ relation to double-check the data, if the data fall into the predicted region in the $\Delta \beta_{\mathrm{X}, \mathrm{O}}-\Delta \alpha_{\mathrm{X}, \mathrm{O}}$ plane, then this burst can be fully interpreted by such a model. Otherwise, the burst does not fall into this category.

The simplest analytical model (Sari et al. 1998) predicts $\beta=p / 2$ for Regime I $\left(\nu>\nu_{\mathrm{c}}\right)$ and $\beta=(p-1) / 2$ for Regime II $\left(\nu_{\mathrm{m}}<\nu<\nu_{\mathrm{c}}\right)$. Detailed numerical calculations (Uhm \& Zhang 2014a) showed that the transition between the two regimes may take several orders of magnitude in observer time. As a result, some "gray zones" with $(p-1) / 2<\beta<p / 2$ are allowed by the model. Therefore, the parameter space between the two closure relation lines defined by the two spectral regimes in the $\alpha-\beta$ plane is allowed by the theory. Data points falling into this gray zone should be regarded as consistent with the model. There are three possibilities: (1) the optical band is in Regime II, while the X-ray band is in the gray zone; (2) the X-ray band is in Regime I, while the optical band is in the gray zone; and (3) both bands are in the gray zone.

For the cases where both the optical and X-ray bands are in the same spectral regime, we demand that the three spectral indices be the same within errors, i.e., $\beta_{\mathrm{O}}=\beta_{\mathrm{OX}}=\beta_{\mathrm{X}}$, where $\beta_{\mathrm{OX}}$ is the spectral index between the optical and X-ray band in the joint spectral energy distribution (SED). ${ }^{15}$ If the two bands are in different spectral regimes, we demand $\beta_{\mathrm{O}}<\beta_{\mathrm{OX}} \leqslant \beta_{\mathrm{X}}$ or $\beta_{\mathrm{O}} \leqslant \beta_{\mathrm{OX}}<\beta_{\mathrm{X}}$.

\footnotetext{
15 In order to obtain $\beta_{\mathrm{OX}}$, we roughly fit the SED from the optical to the X-ray bands. For the optical band, we chose the $\mathrm{R}$ band where the extinction correction is negligible. For the X-rays, we use the Swift XRT data and adopt a typical band $1.5-2 \mathrm{keV}$, where the absorption effect is negligible.
} 

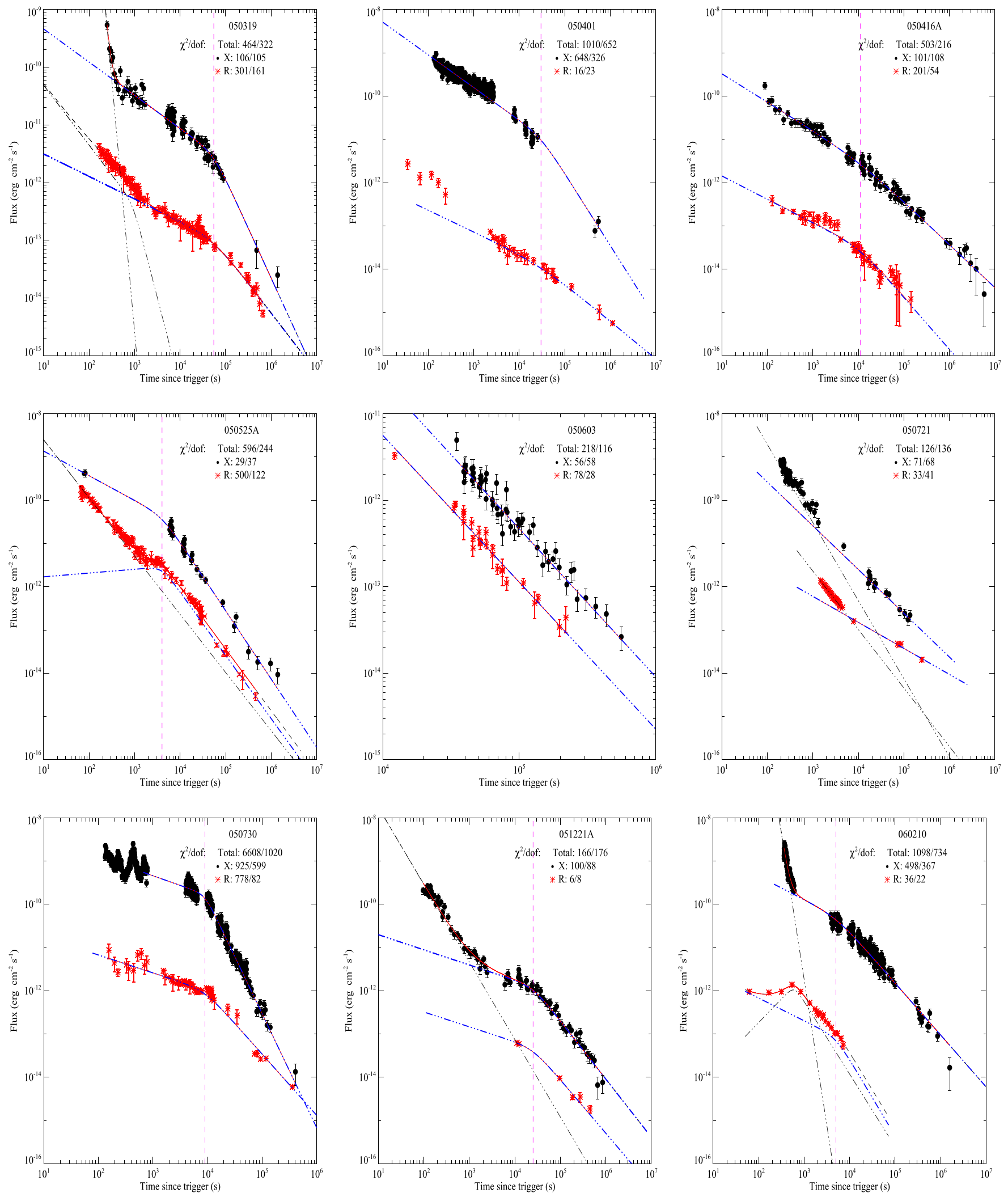

Figure 3. Same as Figure 1, but for the Grade III sample.

\section{COMPARING DATA WITH MODELS}

\subsection{Grading Criteria and Sample Definitions}

With the above preparation, everything is in place for us to systematically compare the broadband data with the external forward shock afterglow models. Based on how badly the data violate the models, we define the following five grades (see also Table 5).

1. Grade I: Both X-ray and optical bands have SPL light curves or BPL light curves with an acceptable achromatic 

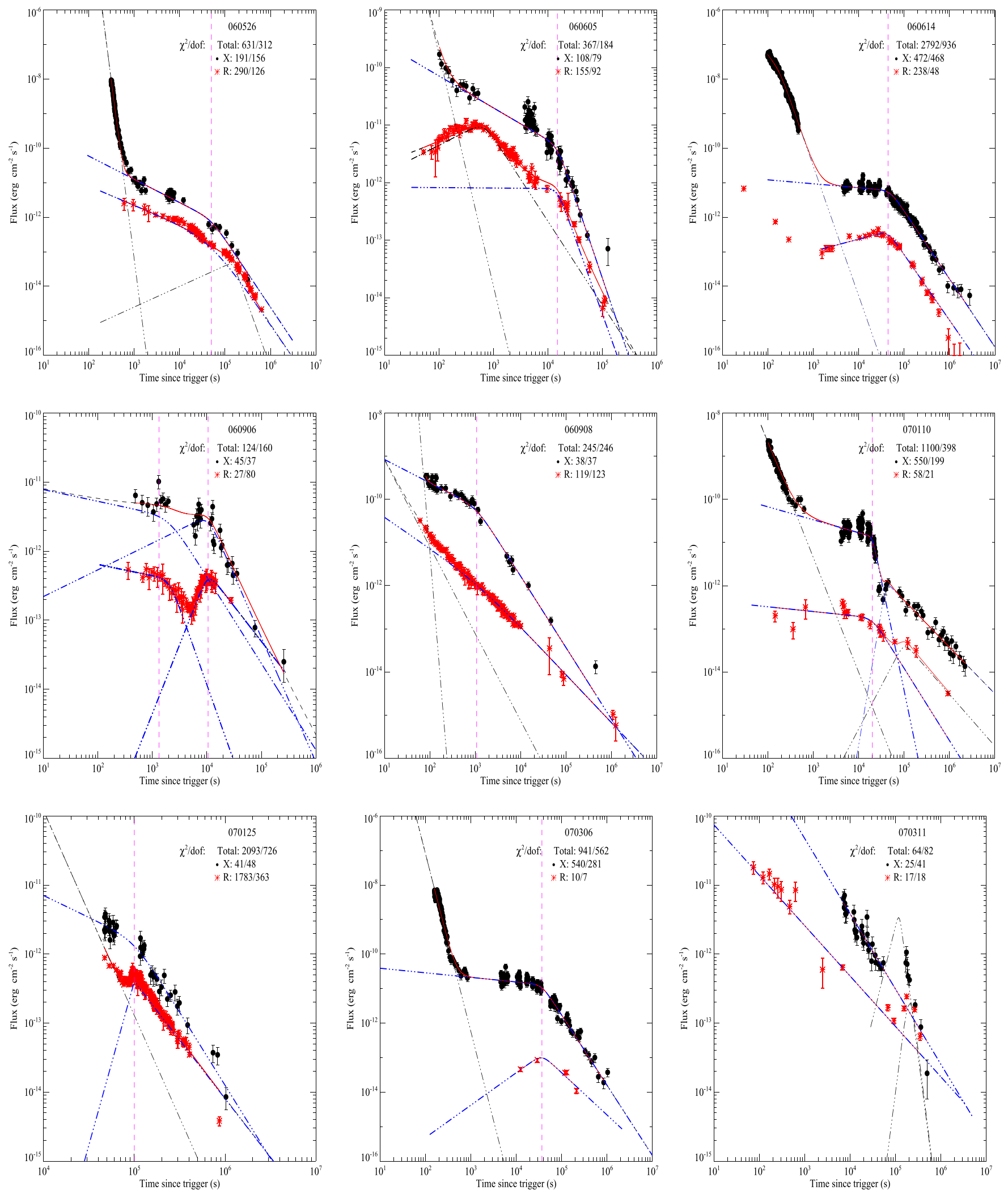

Figure 3. (Continued.)

break. Both bands satisfy closure relations and are selfconsistent (same medium type, $p$ and $q$ values). These are the best examples where the GRB afterglow data abide by the external shock model predictions.
2. Grade II: Some GRBs have a clear break at $t_{\mathrm{b}}$ in one band (e.g., X-rays), but do not have a break in another band (e.g., optical). The missing break is likely due to incomplete observational coverage before or after the 

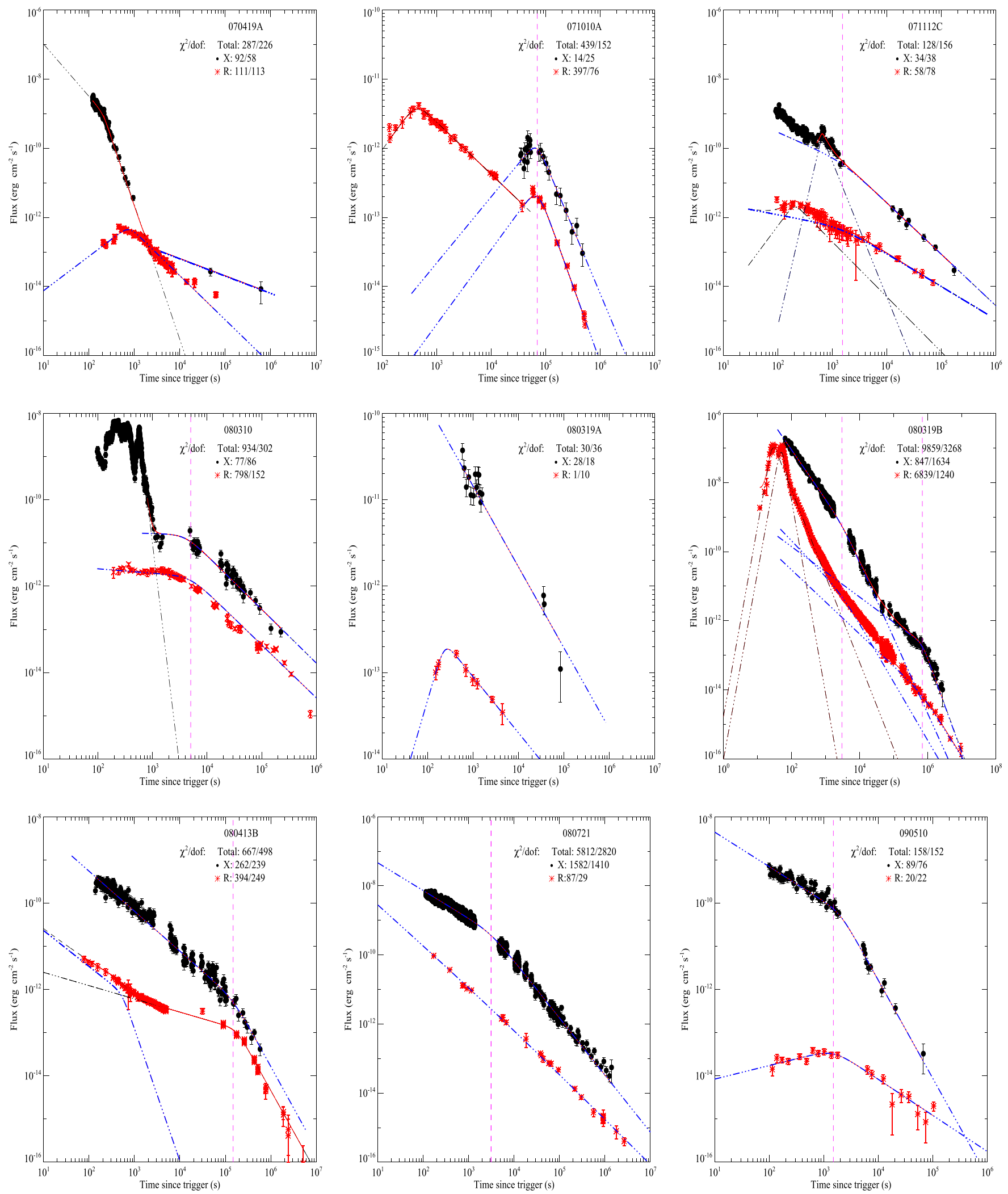

Figure 3. (Continued.)

break. The data are consistent with the hypothesis of an achromatic break, and both bands satisfy closure relations self-consistently. These GRBs are almost as good as Grade I in terms of abiding by the external shock models.
3. Grade III: Both X-ray and optical bands have SPL light curves or BPL light curves with an acceptable achromatic break. However, at least one temporal segment in one band does not satisfy the closure relations 

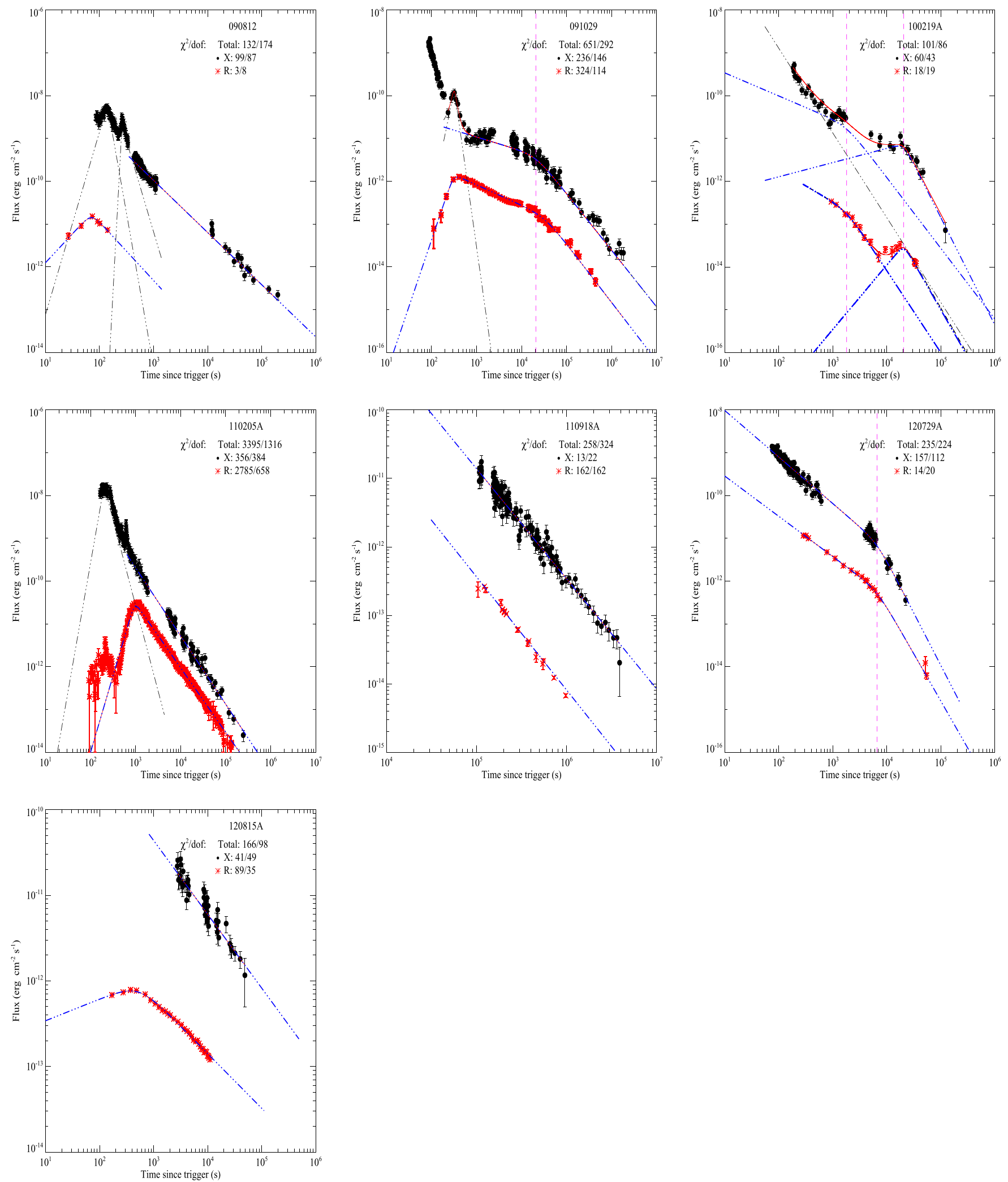

Figure 3. (Continued.)

in a self-consistent manner with respect to other segments or band.

4. Grade IV: This is the Grade II equivalent for Grade III. One band does not have a break, but the data are consistent with the hypothesis of having an achromatic break. At least one temporal segment in one band does not satisfy the closure relations in a self-consistent manner with respect to other segments or band. 

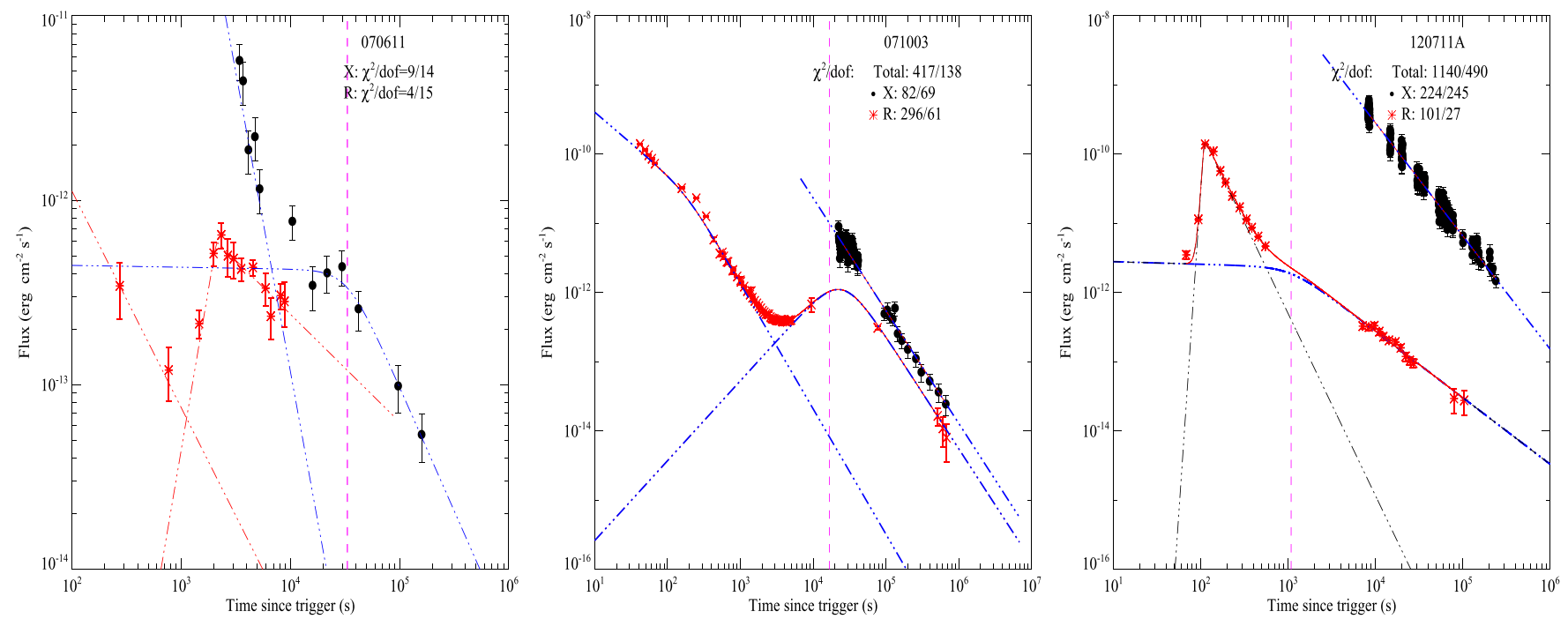

Figure 4. Same as Figure 1, but for the Grade IV sample.
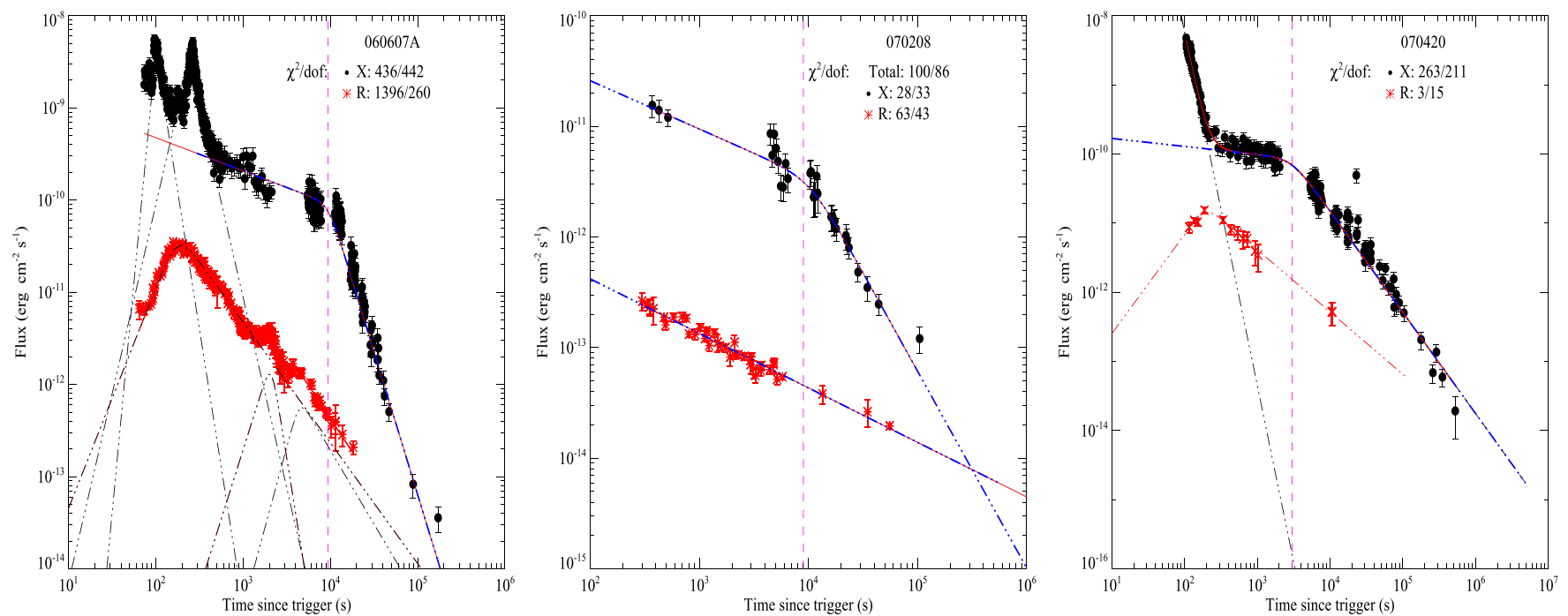

Figure 5. Same as Figure 1, but for the Grade V sample.

5. Grade V: There is clear evidence of chromatic breaks and violation of closure relations. These GRBs cannot be interpreted within the one-component external shock models. ${ }^{16}$

With these five grades, we define three samples.

1. Gold Sample: The GRBs in Grade I and II are defined as the Gold sample GRBs because no observed information violates any predictions of the external shock models.

2. Silver Sample: The GRBs in Grade III and IV are included in this sample. Even though at least one segment or band does not satisfy the closure relations selfconsistently, the basic requirement of achromaticity is not violated. We note that the closure relations are the predictions of the simplest analytical external forward shock models. More complicated models invoking, for example, a structured jet (Rossi et al. 2002; Zhang \&

\footnotetext{
${ }^{16}$ Some of these GRBs may still be interpreted within two-component external shock models with each component dominating one band (e.g., De Pasquale et al. 2009). However, the parameters demanded for the two components are rather contrived
}

Mészáros 2002; Granot \& Kumar 2003; Kumar \& Granot $2003)$ or a circumburst density medium with an arbitrary $k$ value (at least for a certain distance range) predict light curve behaviors that may not fully abide by the simple closure relations. Furthermore, if the GRB engine is longlived and a long-lasting reverse shock outshines the forward shock, a variety of rich light curve behaviors can be generated, which do not follow the simple closure relations (e.g., Uhm et al. 2012; Uhm \& Zhang 2014b). So it is possible that the GRBs in the Silver sample are still consistent with the external shock models.

3. Bad Sample: The GRBs in Grade V violate the basic achromaticity principle of the external shock models and do not abide by the closure relations and therefore cannot be interpreted within the framework of the external shock models.

\subsection{Grading Results}

The 85 well-sampled GRBs in our sample are graded based on the above-defined grading criteria. The GRBs in 
Table 2

Temporal Decay Index $\alpha$ and Spectral Index $\beta$ in Different Afterglow Models

\begin{tabular}{|c|c|c|c|c|c|c|}
\hline CMB & Spectral Regime & $\beta(p)$ & $\alpha(p) / \alpha(p, q)$ & $\alpha(\beta) / \alpha(\beta, q)$ & $\alpha(p) / \alpha(p, q)$ & $\alpha(\beta) / \alpha(\beta, q)$ \\
\hline & & & \multicolumn{2}{|c|}{$p>2$} & \multicolumn{2}{|c|}{$1<p<2$} \\
\hline & \multicolumn{4}{|c|}{ Adiabatic deceleration without energy injection } & & \\
\hline $\begin{array}{l}\text { ISM } \\
\text { Wind }\end{array}$ & $\begin{array}{c}\nu_{\mathrm{m}}<\nu<\nu_{\mathrm{c}} \\
\nu>\nu_{\mathrm{c}} \\
\nu_{\mathrm{m}}<\nu<\nu_{\mathrm{c}} \\
\nu>\nu_{\mathrm{c}}\end{array}$ & $\begin{array}{l}\frac{p-1}{2} \\
\frac{p}{2} \\
\frac{p-1}{2} \\
\frac{p}{2}\end{array}$ & $\begin{array}{l}\frac{3(p-1)}{4} \\
\frac{3 p-2}{4} \\
\frac{3 p-1}{4} \\
\frac{3 p-2}{4}\end{array}$ & $\begin{array}{c}\alpha=\frac{3 \beta}{2} \\
\alpha=\frac{3 \beta-1}{2} \\
\alpha=\frac{3 \beta+1}{2} \\
\alpha=\frac{3 \beta-1}{2}\end{array}$ & $\begin{array}{l}\frac{3(p+2)}{16} \\
\frac{3 p+10}{16} \\
\frac{p+8}{8} \\
\frac{p+6}{8}\end{array}$ & $\begin{aligned} \alpha & =\frac{6 \beta+9}{16} \\
\alpha & =\frac{3 \beta+5}{8} \\
\alpha & =\frac{2 \beta+9}{8} \\
\alpha & =\frac{2 \beta+6}{8}\end{aligned}$ \\
\hline
\end{tabular}

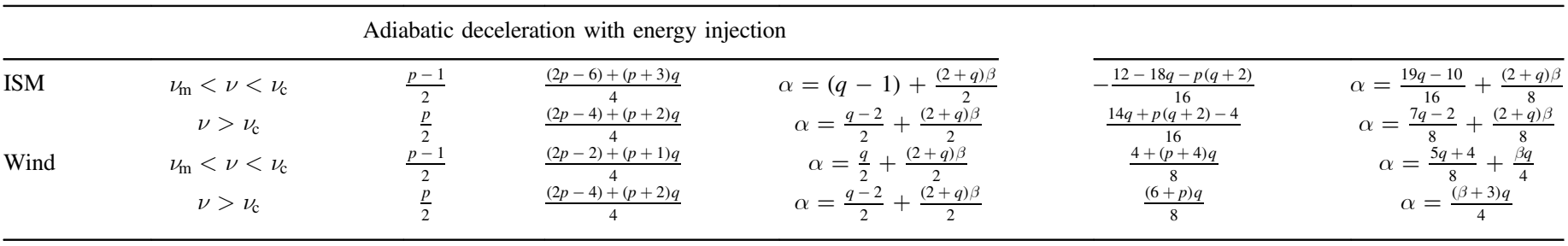

\begin{tabular}{|c|c|c|c|c|c|c|}
\hline \multirow[b]{2}{*}{ ISM } & \multicolumn{4}{|c|}{ Post-jet-break phase without energy injection } & \multirow[b]{2}{*}{$\frac{3(p+6)}{16}$} & \multirow[b]{2}{*}{$\alpha=\frac{3(2 \beta+7)}{16}$} \\
\hline & $\nu_{\mathrm{m}}<\nu<\nu_{\mathrm{c}}$ & $\frac{p-1}{2}$ & $\frac{3 p}{4}$ & $\alpha=\frac{6 \beta+3}{4}$ & & \\
\hline & $\nu>\nu_{\mathrm{c}}$ & $\frac{p}{2}$ & $\frac{3 p+1}{4}$ & $\alpha=\frac{6 \beta+1}{4}$ & $\frac{3 p+22}{16}$ & $\alpha=\frac{3 \beta+11}{8}$ \\
\hline \multirow[t]{3}{*}{ Wind } & $\nu_{\mathrm{m}}<\nu<\nu_{\mathrm{c}}$ & $\frac{p-1}{2}$ & $\frac{3 p+1}{4}$ & $\alpha=\frac{3 \beta+2}{2}$ & $\underline{p+12}$ & $\alpha=\frac{2 \beta+13}{\Omega}$ \\
\hline & $\nu>\nu_{\mathrm{c}}$ & $\underline{p}$ & $\underline{3 p}$ & $\alpha=\frac{3 \beta}{\beta}$ & $p+10$ & $\alpha=\frac{8}{8+5}$ \\
\hline & & & 4 & & 8 & 4 \\
\hline
\end{tabular}

Post-jet-break phase with energy injection

\begin{tabular}{|c|c|c|c|c|c|c|}
\hline ISM & & $\frac{p-1}{\frac{p}{p}}$ & $\frac{p(q+2)-4(1-q)}{4}$ & 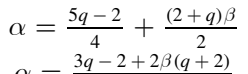 & $\begin{array}{l}\frac{22 q-4+p(q+2)}{16} \\
18 q+4+p(q+2)\end{array}$ & $\begin{array}{l}\alpha=\frac{11 q-2}{8}+\frac{(2+q) \beta}{8} \\
9 q+2+\beta(q+2)\end{array}$ \\
\hline Wind & $\begin{array}{c}\nu>\nu_{\mathrm{c}} \\
\nu_{\mathrm{m}}<\nu<\nu_{\mathrm{c}} \\
\nu>\nu_{\mathrm{c}}\end{array}$ & $\frac{\frac{p}{2}}{\frac{p-1}{2}}$ & $\begin{array}{c}\frac{3 q-2+p(q+2)}{4} \\
\frac{p(q+2)-4(1-q)}{4}\end{array}$ & $\begin{array}{c}\alpha=\frac{4}{\alpha=q+\frac{(2+q) \beta}{2}} \\
\alpha=\frac{\beta(q+2)-2(1-q)}{2}\end{array}$ & $\begin{array}{c}16 \\
\frac{p q+8 q+4}{8} \\
\frac{(p+10) q}{8}\end{array}$ & $\begin{array}{c}\alpha=\frac{8}{\alpha=\frac{1}{2}+\frac{(2 \beta+9) q}{8}} \\
\alpha=\frac{(\beta+5) q}{4}\end{array}$ \\
\hline
\end{tabular}

the five grades are presented in Figures 1-5, respectively. The relevant data of different grades are presented in Tables 1 and 6 .

1. Grade I: As can be seen from Tables 1 and 6 and Figure 1, within errors, 43 of 85 GRBs satisfy the Grade I criteria. Out of 43 GRBs, 13, 8, and 22 GRBs are constrained to have an energy injection break, jet break, and SPL decay, respectively.

2. Grade II: Within errors, two of 85 GRBs fall into this grade (Figure 2).

3. Grade III: There are 34 of 85 GRBs that fall into this grade (Figure 3). Within the sample, 15 and 19 GRBs have SPL and BPL light curves, respectively. GRBs 060906, 080319B, and 100219A have two breaks at different times.

4. Grade IV: Three of 85 GRBs fall into this grade (Figure 4).

5. Grade V: Three of 85 GRBs fall into this grade (Figure 5). Two of them (GRBs 060607A and 070208) show clear chromatic breaks with good temporal coverage in both bands at the break times. One GRB (GRB 070420) shows a chromatic behavior based on the available data and simple model fitting, even though no observational data are available in the optical band at the break time of the X-ray band, so the existence of a break in the optical band (even though very contrived in shape) at the same epoch is not completely ruled out.
Consequently, we get three samples.

1. Gold sample: This sample has 45 of $85 \mathrm{GRBs}$, including $13 / 45,8 / 45$, and $24 / 45$ GRBs satisfying the energy injection, jet break, jet break with energy injection, and SPL decay models, respectively. Among them, 27/45 and $18 / 45$ are consistent with the ISM and wind models, respectively; 17/45, 4/45, and 24/45 GRBs are consistent with being in the same spectral regime, different spectral regimes (X-ray band in regime I and optical band in regime II), and gray zone, respectively. Among the 17 GRBs with the same spectral regime, 15 and two GRBs are consistent with being in the ISM II and wind II spectral regimes, respectively. For the four GRBs with a different spectral regime, all of them are consistent with having an ISM medium.

2. Silver sample: This sample has 37 of $85 \mathrm{GRBs}$, which may (or may not) be interpreted within the more complicated numerical external shock models.

3. Bad sample: Only three of 85 GRBs definitely violate the basic achromaticity principle of the external shock models and therefore belong to the bad sample.

Figure 7 (a) shows $\Delta \beta_{\mathrm{X}, \mathrm{O}}-\Delta \alpha_{\mathrm{X}, \mathrm{O}}$ distributions for the Gold sample. For the energy injection sample, we only used the postbreak segment to remove the $q$ dependence. These are the GRBs that also satisfy the closure relations in all temporal segments. We do not show the closure relation $\alpha-\beta$ plots 


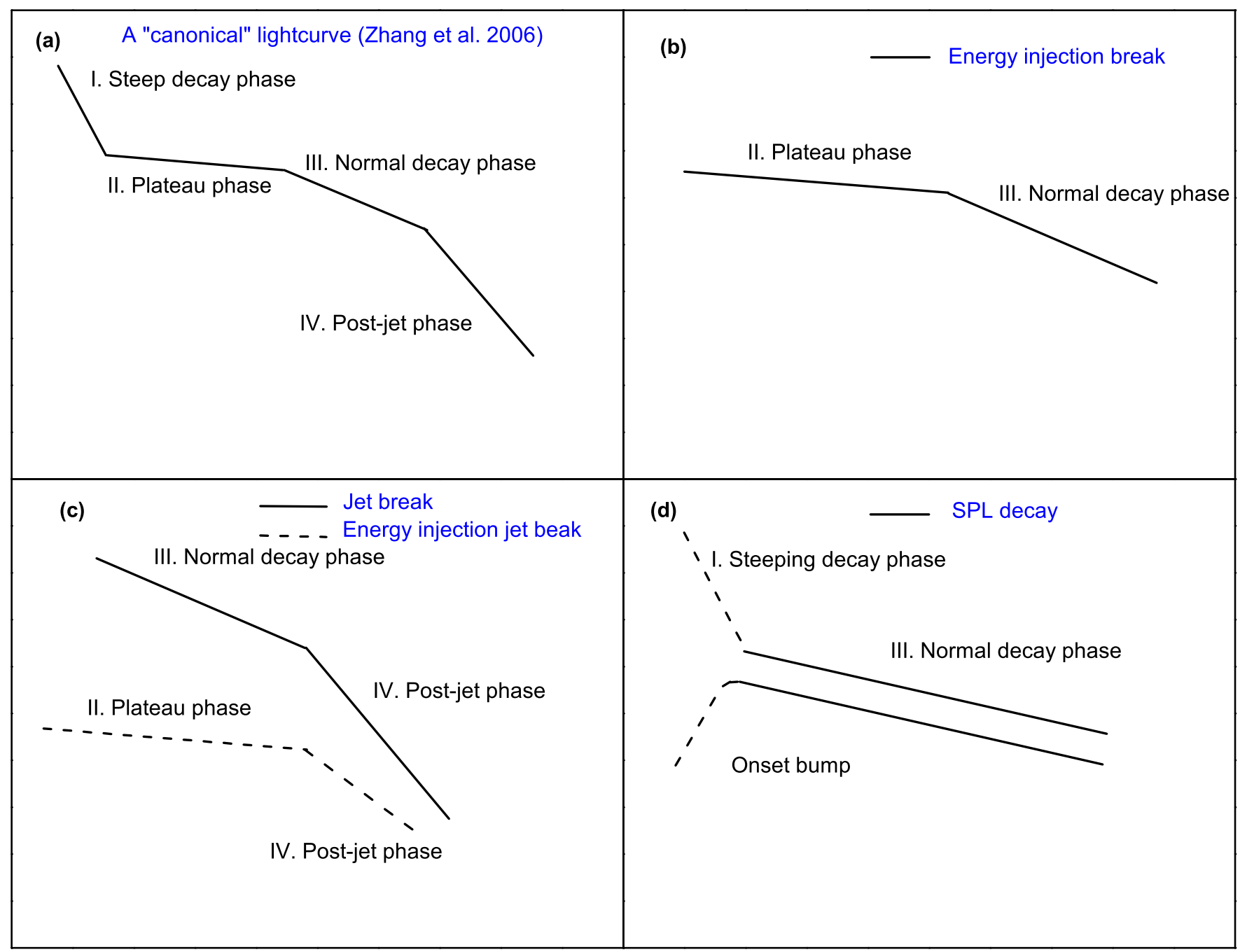

Figure 6. Typical light curve behaviors: (a) the canonical X-ray light curve, reproduced from Zhang et al. (2006), with four characteristic temporal segments marked; (b) the energy injection break case, with a transition from the shallow decay phase (segment II) to the normal decay phase (segment III); (c) the jet break cases without (solid) or with (dashed) energy injection. For the former, it is a transition from the normal decay phase (segment III) to the post-jet-break phase (segment IV); for the latter, both segments have a shallower decay slope; (d) the single power-law (SPL) case. The steep decay phase in the X-ray light curve and the early rising phase in the optical light curve are not included in the analysis.

since the energy injection models have an extra $q$ dependence on the $\alpha$ values. To show the details of how each burst may fall into the model predictions of each model (gray zone included), in Figures 7(b)-(e) we show the $\Delta \beta_{\mathrm{X}, \mathrm{O}}-\Delta \alpha_{\mathrm{X}, \mathrm{O}}$ distributions of those Gold sample GRBs that satisfy the ISM and wind medium models with $p>2$ and $1<p<2$, respectively. The Silver sample GRBs are collected in Figure 7(f). About half of them fall outside the predicted region (red box) defined by the models. Even though some fall into the box, they do not satisfy the closure relations in all of the temporal segments in all energy bands.

\section{STATISTICS OF THE EXTERNAL SHOCK AFTERGLOW MODEL PARAMETERS}

Because the Gold sample (Grade I and II) GRBs comply with the external shock models well, they serve as an excellent sample to study external shock model parameters. The derived external shock parameters of the Gold sample GRBs are presented in Table 6 . We present some statistical properties of these model parameters in this section.

\subsection{Temporal Indices $\alpha$}

Figure 8 shows the distributions of the temporal indices $\alpha$ in different energy bands and different temporal segments. They are all well fitted with Gaussian distributions for each band or temporal segment. For the GRBs having a BPL light curve, the typical $\alpha$ values are $\alpha_{\mathrm{O}, 1}=0.49 \pm 0.45, \alpha_{\mathrm{O}, 2}=1.44 \pm 0.39$, $\alpha_{\mathrm{X}, 1}=0.58 \pm 0.63$, and $\alpha_{\mathrm{X}, 2}=1.50 \pm 0.27$, respectively (Figure 8(a)). For the GRBs with an SPL light curve, one has $\alpha_{\mathrm{O}}=1.26 \pm 0.38$ and $\alpha_{\mathrm{X}}=1.39 \pm 0.26$ (Figure 8(b)). For the BPL sample, we also separate it into the energy injection sample and the jet break sample and perform the statistics. For the energy injection breaks, one has $\alpha_{\mathrm{O}, 1}=0.25 \pm 0.12$, $\alpha_{\mathrm{O}, 2}=1.26 \pm 0.26, \alpha_{\mathrm{X}, 1}=0.30 \pm 0.27$, and $\alpha_{\mathrm{X}, 2}=1.35 \pm$ 0.24 , respectively (Figure $8(\mathrm{c})$ ). For the jet breaks, one has $\alpha_{\mathrm{O}, 1}=0.77 \pm 0.18, \alpha_{\mathrm{O}, 2}=1.66 \pm 0.16, \alpha_{\mathrm{X}, 1}=0.95 \pm 0.16$, and $\alpha_{\mathrm{X}, 2}=1.70 \pm 0.19$, respectively (Figure $8(\mathrm{~d})$ ). Both the prebreak and the postbreak $\alpha$ values in the energy injection sample are systematically shallower than those in the jet break sample. On average, the X-ray light curves are steeper than the optical light curves, consistent with the expectations of the 
Table 3

The $\Delta \alpha_{\mathrm{X}, \mathrm{O}}$ and $\Delta \beta_{\mathrm{X}, \mathrm{O}}$ Values in Different Afterglow Models, $p>2$

\begin{tabular}{|c|c|c|c|c|}
\hline \multicolumn{5}{|c|}{$p>2$} \\
\hline Same Regime ${ }^{\mathrm{a}}$ & \multicolumn{2}{|c|}{ Different Regimes $^{b}$} & \multicolumn{2}{|c|}{ Gray Zone ${ }^{c}$} \\
\hline \multirow[t]{2}{*}{ ISM,Wind } & ISM & Wind & ISM & Wind \\
\hline & & \multicolumn{2}{|c|}{ SPL } & \\
\hline$\Delta \beta_{\mathrm{X}, \mathrm{O}}=0$ & $\Delta \beta_{\mathrm{X}, \mathrm{O}}=\frac{1}{2}$ & $\Delta \beta_{\mathrm{X}, \mathrm{O}}=\frac{1}{2}$ & $\Delta \beta_{\mathrm{X}, \mathrm{O}}=\left(0, \frac{1}{2}\right)$ & $\Delta \beta_{\mathrm{X}, \mathrm{O}}=\left(0, \frac{1}{2}\right)$ \\
\hline \multirow[t]{2}{*}{$\Delta \alpha_{\mathrm{X}, \mathrm{O}}=0$} & $\Delta \alpha_{\mathrm{X}, \mathrm{O}}=\frac{1}{4}$ & $\Delta \alpha_{\mathrm{X}, \mathrm{O}}=-\frac{1}{4}$ & $\Delta \alpha_{\mathrm{X}, \mathrm{O}}=\left(0, \frac{1}{4}\right)$ & $\Delta \alpha_{\mathrm{X}, \mathrm{O}}=\left(-\frac{1}{4}, 0\right)$ \\
\hline & & \multicolumn{2}{|c|}{ Energy injection break } & \\
\hline$\Delta \beta_{\mathrm{X}, \mathrm{O}}=0$ & $\Delta \beta_{\mathrm{X}, \mathrm{O}}=\frac{1}{2}$ & $\Delta \beta_{\mathrm{X}, \mathrm{O}}=\frac{1}{2}$ & $\Delta \beta_{\mathrm{X}, \mathrm{O}}=\left(0, \frac{1}{2}\right)$ & $\Delta \beta_{\mathrm{X}, \mathrm{O}}=\left(0, \frac{1}{2}\right)$ \\
\hline$\Delta \alpha_{1, \mathrm{X}, \mathrm{O}}=0$ & $\Delta \alpha_{1, \mathrm{X}, \mathrm{O}}=\frac{2-q}{4}$ & $\Delta \alpha_{1, \mathrm{X}, \mathrm{O}}=\frac{-2+q}{4}$ & $\Delta \alpha_{1, \mathrm{X}, \mathrm{O}}=\left(0, \frac{2-q}{4}\right)$ & $\Delta \alpha_{1, \mathrm{X}, \mathrm{O}}=\left(\frac{-2+q}{4}, 0\right)$ \\
\hline \multirow[t]{2}{*}{$\Delta \alpha_{2, \mathrm{X}, \mathrm{O}}=0$} & $\Delta \alpha_{2, \mathrm{X}, \mathrm{O}}=\frac{1}{4}$ & $\Delta \alpha_{2, \mathrm{X}, \mathrm{O}}=-\frac{1}{4}$ & $\Delta \alpha_{2, \mathrm{X}, \mathrm{O}}=\left(0, \frac{1}{4}\right)$ & $\Delta \alpha_{2, \mathrm{X}, \mathrm{O}}=\left(-\frac{1}{4}, 0\right)$ \\
\hline & & \multicolumn{2}{|c|}{ Jet break } & \\
\hline$\Delta \beta_{\mathrm{X}, \mathrm{O}}=0$ & $\Delta \beta_{\mathrm{X}, \mathrm{O}}=\frac{1}{2}$ & $\Delta \beta_{\mathrm{X}, \mathrm{O}}=\frac{1}{2}$ & $\Delta \beta_{\mathrm{X}, \mathrm{O}}=\left(0, \frac{1}{2}\right)$ & $\Delta \beta_{\mathrm{X}, \mathrm{O}}=\left(0, \frac{1}{2}\right)$ \\
\hline$\Delta \alpha_{1, \mathrm{X}, \mathrm{O}}=0$ & $\Delta \alpha_{1, \mathrm{X}, \mathrm{O}}=\frac{1}{4}$ & $\Delta \alpha_{1, \mathrm{X}, \mathrm{O}}=-\frac{1}{4}$ & $\Delta \alpha_{1, \mathrm{X}, \mathrm{O}}=\left(0, \frac{1}{4}\right)$ & $\Delta \alpha_{1, \mathrm{X}, \mathrm{O}}=\left(-\frac{1}{4}, 0\right)$ \\
\hline \multirow[t]{2}{*}{$\Delta \alpha_{2, \mathrm{X}, \mathrm{O}}=0$} & $\Delta \alpha_{2, \mathrm{X}, \mathrm{O}}=\frac{1}{4}$ & $\Delta \alpha_{2, \mathrm{X}, \mathrm{O}}=-\frac{1}{4}$ & $\Delta \alpha_{2, \mathrm{X}, \mathrm{O}}=\left(0, \frac{1}{4}\right)$ & $\Delta \alpha_{2, \mathrm{X}, \mathrm{O}}=\left(-\frac{1}{4}, 0\right)$ \\
\hline & & \multicolumn{2}{|c|}{ Energy injection with jet break } & \\
\hline$\Delta \beta_{\mathrm{X}, \mathrm{O}}=0$ & $\Delta \beta_{\mathrm{X}, \mathrm{O}}=\frac{1}{2}$ & $\Delta \beta_{\mathrm{X}, \mathrm{O}}=\frac{1}{2}$ & $\Delta \beta_{\mathrm{X}, \mathrm{O}}=\left(0, \frac{1}{2}\right)$ & $\Delta \beta_{\mathrm{X}, \mathrm{O}}=\left(0, \frac{1}{2}\right)$ \\
\hline$\Delta \alpha_{1, \mathrm{X}, \mathrm{O}}=0$ & $\Delta \alpha_{1, \mathrm{X}, \mathrm{O}}=\frac{2-q}{4}$ & $\Delta \alpha_{1, \mathrm{X}, \mathrm{O}}=\frac{-2+q}{4}$ & $\Delta \alpha_{1, \mathrm{X}, \mathrm{O}}=\left(0, \frac{2-q}{4}\right)$ & $\Delta \alpha_{1, \mathrm{X}, \mathrm{O}}=\left(0, \frac{2-q}{4}\right)$ \\
\hline$\Delta \alpha_{2, \mathrm{X}, \mathrm{O}}=0$ & $\Delta \alpha_{2, \mathrm{X}, \mathrm{O}}=\frac{2-q}{4}$ & $\Delta \alpha_{2, \mathrm{X}, \mathrm{O}}=\frac{-2+q}{4}$ & $\Delta \alpha_{2, \mathrm{X}, \mathrm{O}}=\left(0, \frac{2-q}{4}\right)$ & $\Delta \alpha_{2, \mathrm{X}, \mathrm{O}}=\left(0, \frac{2-q}{4}\right)$ \\
\hline
\end{tabular}

Notes.

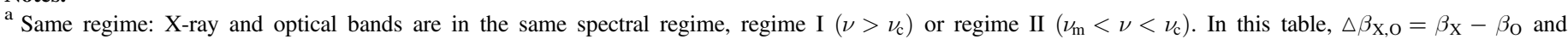
$\Delta \alpha_{\mathrm{X}, \mathrm{O}}=\alpha_{\mathrm{X}}-\alpha_{\mathrm{O}}$. The subscripts " 1 " and " 2 " denote the pre- and postbreak segments for the BPL light curves, respectively.

${ }^{\mathrm{b}}$ Different regimes: X-ray band in regime I $\left(\nu>\nu_{\mathrm{c}}\right)$, and optical band in regime $\mathrm{II}\left(\nu_{\mathrm{m}}<\nu<\nu_{\mathrm{c}}\right)$.

${ }^{c}$ Gray zone: one band or two bands in the gray zone regime I-II.

theoretical models (i.e., the X-ray band is more likely above $\nu_{\mathrm{c}}$ while the optical band is more likely below $\nu_{\mathrm{c}}$ ).

Another self-consistency check is to compare the observed change of decay slope, $\Delta \alpha=\alpha_{2}-\alpha_{1}$, with the model predictions. From the closure relations (Table 2), one can derive these relations:

1. for energy injection breaks:

$$
\begin{aligned}
& \Delta \alpha \\
& \begin{cases}\frac{(1-q)(2+\beta)}{2}, & \text { ISM II }(p>2) \\
\frac{(1-q)(19+2 \beta)}{16}, & \text { ISM II }(1<p<2), \\
\frac{(1-q)(1+\beta)}{2}, & \text { ISM I }(p>2), \\
\frac{(1-q)(7+\beta)}{8}, & \text { wind II }(p>2), \text { wind I }(p>2) \\
\frac{(1-q)(5+2 \beta)}{8}, & \text { wind II }(1<p<2) \\
\frac{(1-q)(3+\beta)}{4}, & \text { wind I }(1<p<2) ;\end{cases}
\end{aligned}
$$

2. for jet breaks:

$$
\Delta \alpha=\left\{\begin{array}{l}
\frac{3}{4}, \quad \text { ISM I and II }(p>2 \text { and } 1<p<2) \\
\frac{1}{2}, \text { wind I and II }(p>2 \text { and } 1<p<2)
\end{array}\right.
$$

3. for jet breaks with energy injection:

$$
\Delta \alpha= \begin{cases}\frac{(q+2)}{4}, & \text { ISM II }(p>2), \\ \frac{(3 q+6)}{16}, & \text { ISM I II }(p>2 \text { and } 1<p<2) \\ \frac{q}{2}, & \text { wind II and wind I } \\ & (p>2 \text { and } 1<p<2) .\end{cases}
$$

Figure 9 shows a comparison between the observed $\Delta \alpha_{\text {obs }}$ and the theoretically predicted $\Delta \alpha_{\text {th }}$ for each GRB derived from the measured $\beta$ and $q$ values using the corresponding closure relations. One can see that the two are consistent with each other.

Figure 10 displays the observed $\Delta \alpha$ distributions of various samples. For the Gold sample, the $\Delta \alpha$ distributions of optical and X-ray data are consistent with each other, i.e., $\Delta \alpha_{\mathrm{O}}=0.94 \pm 0.23$ and $\Delta \alpha_{\mathrm{X}}=0.88 \pm 0.28$ (Figure 10(a)). 
Table 4

The $\Delta \alpha_{\mathrm{X}, \mathrm{O}}$ and $\Delta \beta_{\mathrm{X}, \mathrm{O}}$ Values in Different Afterglow Models, $1<p<2$

\begin{tabular}{lccccc}
\hline \hline & & & & \\
\hline Same Regime $^{\mathrm{a}}$ & & Different Regimes $^{\mathrm{b}}$ & & & Gray Zone $^{\mathrm{c}}$ \\
ISM,Wind & ISM & Wind & & ISM & Wind
\end{tabular}

SPL

\begin{tabular}{|c|c|c|c|c|}
\hline$\Delta \beta_{\mathrm{X}, \mathrm{O}}=0$ & $\Delta \beta_{\mathrm{X}, \mathrm{O}}=\frac{1}{2}$ & $\Delta \beta_{\mathrm{X}, \mathrm{O}}=\frac{1}{2}$ & $\Delta \beta_{\mathrm{X}, \mathrm{O}}=\left(0, \frac{1}{2}\right)$ & $\Delta \beta_{\mathrm{X}, \mathrm{O}}=\left(0, \frac{1}{2}\right)$ \\
\hline$\Delta \alpha_{\mathrm{X}, \mathrm{O}}=0$ & $\Delta \alpha_{\mathrm{X}, \mathrm{O}}=\frac{1}{2}$ & $\Delta \alpha_{\mathrm{X}, \mathrm{O}}=\frac{1}{4}$ & $\Delta \alpha_{\mathrm{X}, \mathrm{O}}=\left(0, \frac{1}{2}\right)$ & $\Delta \alpha_{\mathrm{X}, \mathrm{O}}=\left(0, \frac{1}{4}\right)$ \\
\hline
\end{tabular}

\begin{tabular}{lcccc}
\hline & \multicolumn{2}{c}{ Energy injection break } & & \multicolumn{1}{c}{$c$} \\
\hline$\Delta \beta_{\mathrm{X}, \mathrm{O}}=0$ & $\Delta \beta_{\mathrm{X}, \mathrm{O}}=\frac{1}{2}$ & $\Delta \beta_{\mathrm{X}, \mathrm{O}}=\frac{1}{2}$ & $\Delta \beta_{\mathrm{X}, \mathrm{O}}=\left(0, \frac{1}{2}\right)$ & $\Delta \beta_{\mathrm{X}, \mathrm{O}}=\left(0, \frac{1}{2}\right)$ \\
$\Delta \alpha_{1, \mathrm{X}, \mathrm{O}}=0$ & $\Delta \alpha_{1, \mathrm{X}, \mathrm{O}}=\frac{2-q}{4}$ & $\Delta \alpha_{1, \mathrm{X}, \mathrm{O}}=\frac{-2+q}{4}$ & $\Delta \alpha_{1, \mathrm{X}, \mathrm{O}}=\left(0, \frac{2-q}{4}\right)$ & $\Delta \alpha_{1, \mathrm{X}, \mathrm{O}}=\left(\frac{-2+q}{4}, 0\right)$ \\
$\Delta \alpha_{2, \mathrm{X}, \mathrm{O}}=0$ & $\Delta \alpha_{2, \mathrm{X}, \mathrm{O}}=\frac{1}{2}$ & $\Delta \alpha_{2, \mathrm{X}, \mathrm{O}}=\frac{1}{4}$ & $\Delta \alpha_{2, \mathrm{X}, \mathrm{O}}=\left(0, \frac{1}{2}\right)$ & $\Delta \alpha_{2, \mathrm{X}, \mathrm{O}}=\left(0, \frac{1}{4}\right)$ \\
\hline
\end{tabular}

\begin{tabular}{|c|c|c|c|c|}
\hline \multirow[b]{2}{*}{$\begin{array}{l}\Delta \beta_{\mathrm{X}, \mathrm{O}}=0 \\
\Delta \alpha_{1, \mathrm{X}, \mathrm{O}}=0 \\
\Delta \alpha_{2, \mathrm{X}, \mathrm{O}}=0\end{array}$} & \multirow[b]{2}{*}{$\begin{aligned} \Delta \beta_{\mathrm{X}, \mathrm{O}} & =\frac{1}{2} \\
\Delta \alpha_{1, \mathrm{X}, \mathrm{O}} & =\frac{1}{2} \\
\Delta \alpha_{2, \mathrm{X}, \mathrm{O}} & =\frac{1}{4}\end{aligned}$} & \multicolumn{2}{|c|}{ Jet break } & \multirow[b]{2}{*}{$\begin{array}{c}\Delta \beta_{\mathrm{X}, \mathrm{O}}=\left(0, \frac{1}{2}\right) \\
\Delta \alpha_{1, \mathrm{X}, \mathrm{O}}=\left(0, \frac{1}{4}\right) \\
\Delta \alpha_{2, \mathrm{X}, \mathrm{O}}=\left(-\frac{1}{4}, 0\right)\end{array}$} \\
\hline & & $\begin{array}{c}\Delta \beta_{\mathrm{X}, \mathrm{O}}=\frac{1}{2} \\
\Delta \alpha_{1, \mathrm{X}, \mathrm{O}}=\frac{1}{4} \\
\Delta \alpha_{2, \mathrm{X}, \mathrm{O}}=-\frac{1}{4}\end{array}$ & $\begin{aligned} \Delta \beta_{\mathrm{X}, \mathrm{O}} & =\left(0, \frac{1}{2}\right) \\
\Delta \alpha_{1, \mathrm{X}, \mathrm{O}} & =\left(0, \frac{1}{2}\right) \\
\Delta \alpha_{2, \mathrm{X}, \mathrm{O}} & =\left(0, \frac{1}{4}\right)\end{aligned}$ & \\
\hline & & \multicolumn{2}{|c|}{ Energy injection with jet break } & \\
\hline
\end{tabular}

Notes.

${ }^{a}$ Same regime: X-ray and optical bands are in the same spectral regime, regime I $\left(\nu>\nu_{\mathrm{c}}\right)$ or regime II $\left(\nu_{\mathrm{m}}<\nu<\nu_{\mathrm{c}}\right)$. In this table, $\Delta \beta_{\mathrm{X}, \mathrm{O}}=\beta_{\mathrm{X}}-\beta_{\mathrm{O}}$ and $\Delta \alpha_{\mathrm{X}, \mathrm{O}}=\alpha_{\mathrm{X}}-\alpha_{\mathrm{O}}$. The subscripts "1" and "2" denote the pre- and postbreak segments for the BPL light curves, respectively.

${ }^{\mathrm{b}}$ Different regimes: X-ray band in regime I $\left(\nu>\nu_{\mathrm{c}}\right)$, and optical band in regime $\mathrm{II}\left(\nu_{\mathrm{m}}<\nu<\nu_{\mathrm{c}}\right)$.

${ }^{\mathrm{c}}$ Gray zone: one band or two bands in the gray zone regime I-II.

Furthermore, the $\Delta \alpha$ values of both bands in subgroups (energy injection breaks and jet breaks) are also consistent with each other: $\Delta \alpha_{\mathrm{O}}=1.05 \pm 0.17$ and $\Delta \alpha_{\mathrm{X}}=1.10 \pm 0.21$ for the energy injection breaks, and $\Delta \alpha_{\mathrm{O}}=0.75 \pm 0.22$ and $\Delta \alpha_{\mathrm{X}}=0.75 \pm 0.22$ for the jet breaks (Figure 10(b)). The Silver sample, on the other hand, shows a poorer statistical behavior (Figures 10(c) and (d)).

\subsection{Spectral Indices $\beta$}

Figure 11 shows the spectral index distributions for the Gold sample. In general, the distributions can be fitted with Gaussian functions. For the global sample, one has $\beta_{\mathrm{O}}=0.70 \pm 0.15$ and $\beta_{\mathrm{X}}=0.98 \pm 0.15$ (Figure $11(\mathrm{a})$ ). In the Gold sample, 17 of 45 GRBs have both the optical and X-ray bands in the same spectral regime. One has $\beta_{\mathrm{O}}=0.77 \pm 0.19$ and $\beta_{\mathrm{X}}=0.89 \pm 0.15$, which are consistent with each other (Figure $11(\mathrm{~b})$ ). The remaining 28 of 45 GRBs are identified to have X-ray and optical bands separated by a cooling break. The results show $\beta_{\mathrm{O}}=0.68 \pm 0.18, \beta_{\mathrm{X}}=1.01 \pm 0.14$, with $\Delta \beta=\beta_{\mathrm{X}}-\beta_{\mathrm{O}}=0.37 \pm 0.18$, which is consistent with the theoretically expected value $0<\Delta \beta \leqslant 0.5$ (Figure $11(\mathrm{c})$ ).

We investigate the $\beta$ distributions in different types of light curves. For the energy injection sample, one has $\beta_{\mathrm{O}}=0.78 \pm 0.12$ and $\beta_{\mathrm{X}}=1.01 \pm 0.13$ (Figure $11(\mathrm{~d})$ ); for the jet break sample, one has $\beta_{\mathrm{O}}=0.59 \pm 0.11$ and $\beta_{\mathrm{X}}=0.97 \pm 0.08$ (Figure $11(\mathrm{e})$ ); and for the SPL sample, one has $\beta_{\mathrm{O}}=0.74 \pm 0.24 \quad$ and $\quad \beta_{\mathrm{X}}=0.95 \pm 0.19$ (Figure 11(f)).

We also investigate the $\beta$ distributions in different ambient medium types. For the ISM model (27/45 GRBs), one has $\beta_{\mathrm{O}}=0.72 \pm 0.21$ and $\beta_{\mathrm{X}}=0.98 \pm 0.10$ (Figure $11(\mathrm{~g})$ ); for the wind model (18/45 GRBs), one has $\beta_{\mathrm{O}}=0.70 \pm 0.10$ and $\beta_{\mathrm{X}}=1.00 \pm 0.20$ (Figure $\left.11(\mathrm{~h})\right)$. The ISM model is more favored than the wind model, which is consistent with the previous results (e.g., Panaitescu \& Kumar 2002; Yost et al. 2003; Zhang et al. 2006; Schulze et al. 2011).

\subsection{Electron Spectral Index $p$}

Figure 12 shows the distributions of the electron spectral index $p$ of the Gold sample. It has a Gaussian distribution with $p=2.33 \pm 0.48$ (Figure 12(a)), which is very consistent with the typical value of $p$ for relativistic shocks due to first-order Fermi acceleration (e.g., Achterberg et al. 2001; Ellison \& Double 2002). It also has a wide distribution, which is consistent with previous studies (e.g., Shen et al. 2006; Liang et al. 2007, 2008; Curran et al. 2010).

The $p$ distributions in different subsamples are also generally consistent with each other. Within the Gold sample, those GRBs with optical and X-ray bands in the same spectral regime have $p=2.58 \pm 0.39$, whereas those with optical and X-ray bands in different spectral regimes have $p=2.17 \pm 0.44$ (Figure 12(a)). For the three light curve subsamples, one has 

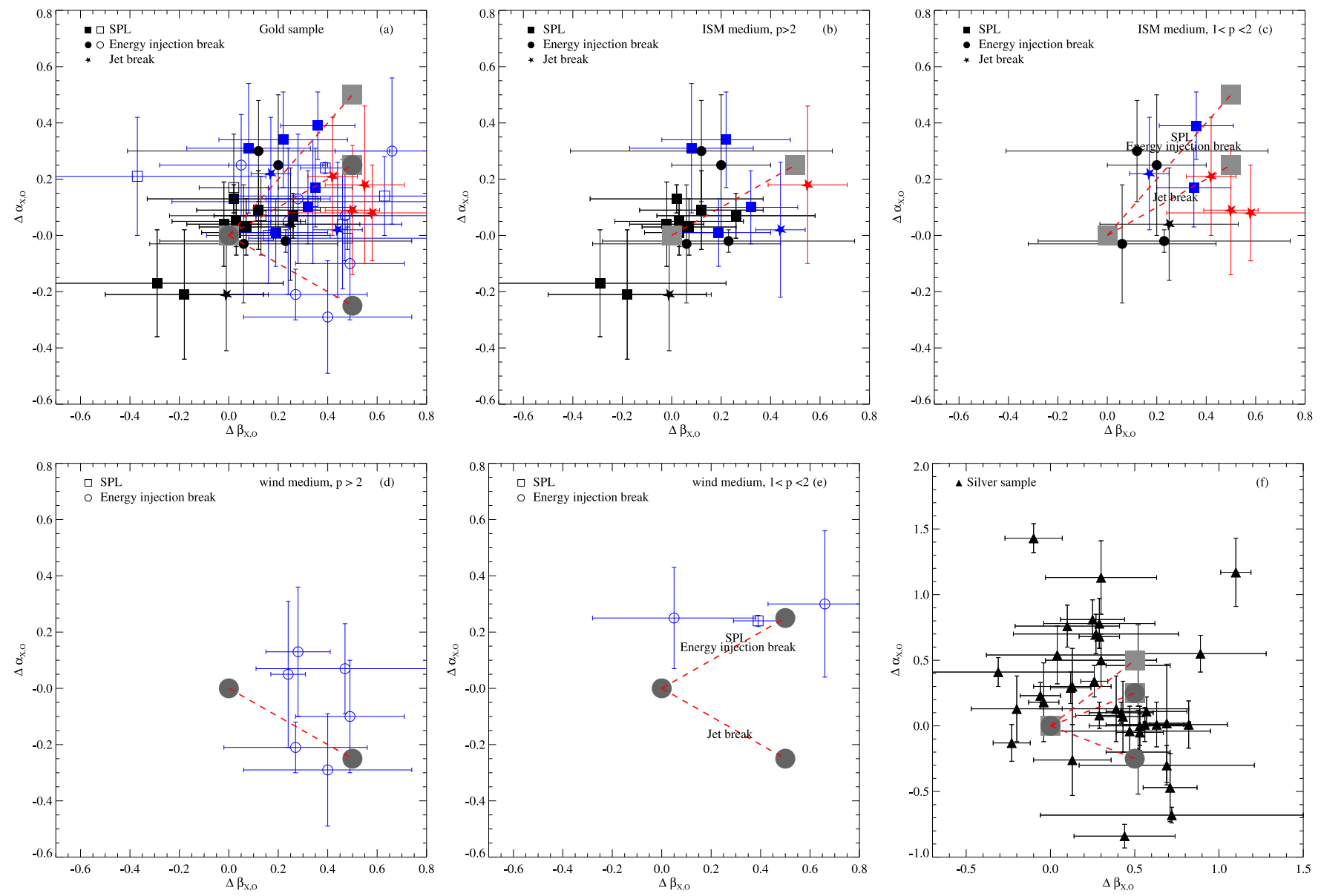

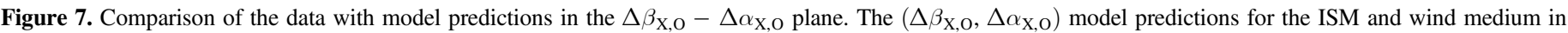

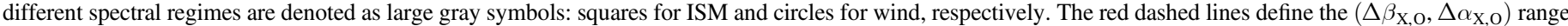

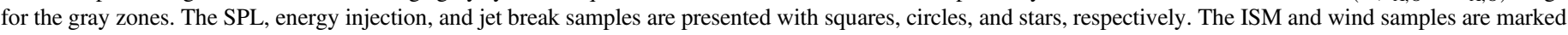

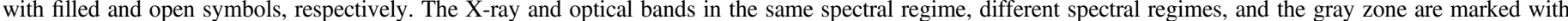

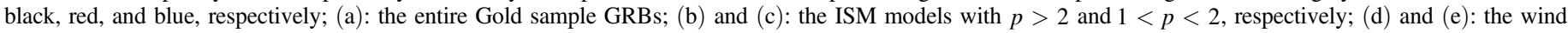
models with $p>2$ and $1<p<2$, respectively; (f): the Silver sample GRBs (denoted as triangles).

$p=2.34 \pm 0.38$ for the energy injection break sample, $p=$ $1.91 \pm 0.37$ for the jet break sample, and $p=2.48 \pm 0.47$ for the SPL sample, respectively (Figure 12(b)). For the two medium type models, one has $p=2.43 \pm 0.57$ for the ISM model and $p=2.28 \pm 0.33$ for the wind model, respectively (Figure 12(c)).

\subsection{Break Time $t_{b}$}

Figure 13 shows the distributions of the observed achromatic break times, $t_{\mathrm{b}}$. The global distribution in the Gold sample gives $\log \left(t_{\mathrm{b}} / \mathrm{ks}\right)=(3.8 \pm 0.9)$. Separating the energy injection sample and jet break sample, one has $\log \left(t_{\mathrm{b}} / \mathrm{ks}\right)=(3.6 \pm 1.9)$ for the energy injection break sample and $\log \left(t_{\mathrm{b}} / \mathrm{ks}\right)=$ $(3.9 \pm 0.7)$ for the jet break sample. The energy injection end (which depends on central engine) is on average earlier than the jet break time (which depends on the geometry of the jet). The distribution of the energy injection break time is wider than the jet break time distribution.

\subsection{Energy Injection Parameter q}

Within the Gold sample, 13 of 45 GRBs show an energy injection type break, either an energy injection break or a jet break with energy injection. Among them, 4/13 and 9/13 GRBs satisfy the ISM and wind model, respectively. The distributions of the energy injection parameter $q$ of various samples are shown in Figure 14. The global sample has $q=0.22 \pm 0.11$. The ISM and wind models have $q=0.20 \pm 0.12$ and $q=0.23 \pm 0.13$, respectively, which are consistent with each other.

\subsection{Shock Magnetic Field Equipartition Factor $\epsilon_{B}$}

Among the derived shock parameters, the magnetic field equipartition factor $\epsilon_{B}$ is of special interest. If the shock simply compresses the upstream magnetic field, then the expected $\epsilon_{B}$ is low, of the order of $10^{-6}-10^{-7}$. If, however, various plasma instabilities are playing a role to amplify the magnetic fields (e.g., Medvedev \& Loeb 1999; Nishikawa et al. 2009), one would expect a relatively large $\epsilon_{B}$, as high as 0.1 . Early afterglow modeling (e.g., Wijers \& Galama 1999; Panaitescu \& Kumar 2001, 2002; Yost et al. 2003) derived a relatively large $\epsilon_{B}$, with a typical value of $\sim 0.01$. On the other hand, modeling of gigaelectronvolt emission in several Fermi/LATdetected GRBs led to the suggestion that $\epsilon_{B}$ should be relatively low, at least for some GRBs (Kumar \& Barniol Duran 2009, 2010). Santana et al. (2014) derived $\epsilon_{B}$ for a large sample of GRBs and derived a medium value of $10^{-5}$. 
Table 5

Criteria for GRB Grades

\begin{tabular}{|c|c|c|c|}
\hline Grades & Light Curve Behavior & Closure relation & Fraction \\
\hline Grade I & Achromatic break or SPL decay in both bands & Yes & $43 / 85$ \\
\hline Grade II & Break missing in one band, consistent with being achromatic & Yes & $2 / 85$ \\
\hline Grade III & Achromatic break or SPL decay in both bands & No & $34 / 85$ \\
\hline Grade IV & Break missing in one band, consistent with being achromatic & No & $3 / 85$ \\
\hline Grade V & Chromatic & No & $3 / 85$ \\
\hline
\end{tabular}

With our Gold sample, we can constrain $\epsilon_{B}$ independently. Even though in most GRBs $\epsilon_{B}$ cannot be constrained due to the degeneracy of the data, one can still place interesting upper limits on $\epsilon_{B}$ based on the medium type and spectral regime of the GRBs. For example, in the ISM model, $\nu_{\mathrm{c}}$ decreases with time. For a regime II $\left(\nu_{\mathrm{m}}<\nu<\nu_{\mathrm{c}}\right) \mathrm{GRB}$, the last data point in the light curve would set a lower limit on $\nu_{\mathrm{c}}$ at that epoch and, hence, an upper limit on $\epsilon_{B}$. Similarly, in the wind model, $\nu_{\mathrm{c}}$ increases with time. For a regime II GRB, the first data point in the light curve would set a lower limit on $\nu_{\mathrm{c}}$ at that point and, hence, an upper limit on $\epsilon_{B}$.

In the appendix, we present expressions of $\nu_{\mathrm{m}}, \nu_{\mathrm{c}}$, and $F_{\nu, \max }$

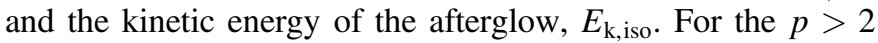
cases, we adopt the formalism of previous works (Zhang et al. 2007a; Gao et al. 2013; Lü \& Zhang 2014). New expressions for the $1<p<2$ regime are also presented following Gao et al. (2013). We then derive the expressions of $\epsilon_{B}$ in various models, Equations (18), (21), (32), and (35), which are used to constrain $\epsilon_{B}$.

The derived upper limits of $\epsilon_{B}$ are presented in Figure 15, with other parameters fixed as $\epsilon_{e}=0.1, n=1$, or $A_{*}=1$. One can see that in general these upper limits point toward a relatively low $\epsilon_{B}$ value. In some cases for the ISM model, the upper limits are even lower than $10^{-5}$. These results are consistent with the findings of Kumar \& Barniol Duran (2009, 2010), Barniol Duran (2014), and Santana et al. (2014).

\subsection{Energetics}

The isotropic $\gamma$-ray energy $E_{\gamma \text {,iso }}$ is calculated as

$$
E_{\gamma, \text { iso }}=\frac{4 \pi D_{\mathrm{L}}^{2} S_{\gamma} k}{1+z},
$$

where $S_{\gamma}$ is the gamma-ray fluence in the BAT band, $D_{\mathrm{L}}$ is the luminosity distance of the source at redshift $z$, and the parameter $k$ is a factor to correct the observed $\gamma$-ray energy in a given bandpass to a broad band (e.g., $1-10^{4} \mathrm{keV}$ in the rest frame) with the observed GRB spectra (Bloom et al. 2001). It is well known that a typical GRB spectrum is well fitted with the so-called Band function (Band et al. 1993). If the Band parameters are measured for a burst, these parameters are used to derive the $k$ parameter. However, owing to the narrowness of the Swift/BAT band, the spectra of many Swift GRBs in our sample are adequately fitted with an SPL, $N \propto E^{-\Gamma}$, so the Band parameters are not well constrained. For these GRBs, we use an empirical relation between $E_{p}$ and the BAT-band photon index $\Gamma$ (Zhang et al. 2007b; Sakamoto et al. 2009; Virgili et al. 2012) to estimate $E_{p}$. Taking typical values of the photon indices $\alpha=-1.1$ and $\beta=-2.2$ (Preece et al. 2000; Kaneko et al. 2006), we can derive the $E_{\gamma \text {,iso }}$ values of the GRBs with redshift measurements in our Gold sample, which range from $10^{51}$ to $10^{55} \mathrm{erg}$, with a typical value of $\log \left(E_{\gamma, \text { iso }} / \mathrm{erg}\right)=$ $53.15 \pm 0.69$ (Figure 16(a)).

The isotropic kinetic energy of the afterglow $E_{\mathrm{K} \text {,iso }}$ can be derived from the afterglow data. In general, broadband modeling is needed to precisely measure $E_{\mathrm{K} \text {,iso }}$ (Panaitescu \& Kumar 2001, 2002). Most GRBs do not have adequate data to perform such an analysis. More conveniently, one may use the $\mathrm{X}$-ray data only to constrain $E_{\mathrm{K} \text {,iso }}$ because the X-ray band is usually above $\nu_{\mathrm{c}}$, so the X-ray flux does not depend on the ambient density and only weakly depends on $\epsilon_{e}$ (Kumar 1999; Freedman \& Waxman 2001; Berger et al. 2003; LloydRonning \& Zhang 2004; Zhang et al. 2007a); see appendix for detailed derivations. For the cases with energy injection, $E_{\mathrm{K} \text {,iso }}$ is a function of time during the energy injection phase. Following Zhang et al. (2007a), we calculate $E_{\mathrm{K} \text {,iso }}$ at two different epochs, one at the break time $t_{\mathrm{b}}$, when energy injection is over, and another at a putative deceleration time $t_{\mathrm{dec}} \sim \max \left(60 \mathrm{~s}, T_{90}\right)$. We use the X-ray flux at $t_{\mathrm{b}}$ to derive $E_{\mathrm{K}, \text { end }}$ and then derive $E_{\mathrm{K}, \mathrm{dec}}=E_{\mathrm{K} \text {,end }}\left(t_{\mathrm{dec}} / t_{\mathrm{b}}\right)^{1-q}$. The total injected energy is calculated as $E_{\mathrm{K}, \text { inj }}=E_{\mathrm{K} \text {,end }}-E_{\mathrm{K} \text {,dec }}$. Based on our constraints on $\epsilon_{B}$, we take $\epsilon_{B}=10^{-5}$ for all of the GRBs in our calculations. Other parameters are taken as typical values: $\epsilon_{e}=0.1, n=1$ or $A_{*}=1$, and $Y=1$.

In Figure 16, we present several statistical results of the $E_{\mathrm{K} \text {,iso }}$ calculations. For the GRBs with energy injection, the distributions are $\log \left(E_{\mathrm{K}, \text { end }} / \mathrm{erg}\right)=54.99 \pm 0.86$ (Figure 16(b)), log $\left(E_{\mathrm{K}, \text { inj }} / \mathrm{erg}\right)=54.95 \pm 0.61 \quad$ (Figure $\left.16(\mathrm{c})\right)$, and $\log$ $\left(E_{\mathrm{K}, \mathrm{dec}} / \mathrm{erg}\right)=53.29 \pm 0.45$ (Figure 16(d)). For the entire Gold sample, one has $\log \left(E_{\mathrm{K}, \mathrm{dec}} / \mathrm{erg}\right)=54.66 \pm 1.18$ (Figure $16(\mathrm{~d}))$. It is interesting to see that the energetics of the energy injection sample reach a level similar to the no-energy-injection sample after the energy injection is over. Clear correlations are found among different energy components: $E_{\mathrm{K} \text {,end }}-E_{\mathrm{K} \text {,inj }}$ relation $E_{\mathrm{K}, \text { inj, } 52}=0.69 E_{\mathrm{K}, \text { end,52 }}^{1.02 \pm 02}$ (Figure $17(\mathrm{a})), \quad E_{\mathrm{K}, \text { dec }}-E_{\mathrm{K}, \text { inj }} \quad$ relation $\quad E_{\mathrm{K}, \text { inj,52 }}=41.7$ $E_{\mathrm{K}, \mathrm{dec}, 52}^{0.76 \pm 0.20}($ Figure $17(\mathrm{~b})), E_{\gamma, \text { iso }}-E_{\mathrm{K} \text {, end }}$ relation $E_{\mathrm{K}, \text { end }, 52}=$ $476.3 E_{\gamma, \text { iso }, 52}^{0.53 \pm 0.23}$ (Figure $17(\mathrm{c})$ ), $E_{\gamma \text {, iso }}-E_{\mathrm{K}, \mathrm{dec}}$ relations in the entire Gold sample, $E_{\mathrm{K}, \mathrm{dec}, 52}=56.2 E_{\gamma, \text { iso,52 }}^{0.93 \pm 0.19}($ Figure $17(\mathrm{~d}))$, in the energy injection sample, $E_{\mathrm{K}, \mathrm{dec}, 52}=8.9 E_{\gamma, \text { iso, } 52}^{1.10 \pm 0.29}$ (Figure 17(e)), and in the no-energy-injection sample, $E_{\mathrm{K}, \mathrm{dec}, 52}=316.2 E_{\gamma, \text { iso,52 }}^{0.55 \pm 0.21} \quad($ Figure $17(\mathrm{f}))$, respectively. In particular, the $E_{\mathrm{K}, \mathrm{inj}, 52}=41.7 E_{\mathrm{K}, \mathrm{dec}, 52}^{0.76 \pm 0.20}$ correlation suggests a substantial energy injection during the shallow decay phase for most GRBs. 
Table 6

Gold Sample GRBs and Their Derived Parameters

\begin{tabular}{|c|c|c|c|c|c|c|c|c|c|c|c|c|c|}
\hline GRB & $z$ & Optical $^{\mathrm{a}}$ & $X$-ray ${ }^{a}$ & $p$ & $q$ & $\epsilon_{B}^{\mathrm{b}}$ & $E_{\gamma, \text { iso }}{ }^{\mathrm{c}}$ & $E_{\mathrm{K}, \text { end }}{ }^{\mathrm{c}}$ & $E_{\mathrm{K}, \mathrm{in}}{ }^{\mathrm{c}}$ & $E_{\mathrm{K}, \mathrm{dec}}{ }^{\mathrm{c}}$ & $\eta_{\gamma, \mathrm{dec}}{ }^{\mathrm{d}}$ & $\eta_{\gamma, \text { end }}{ }^{\mathrm{d}}$ & Type $^{\mathrm{e}}$ \\
\hline 050408 & 1.24 & windII & $\begin{array}{l}\text { windI- } \\
\text { windII }\end{array}$ & $\begin{array}{c}2.12 \\
\pm 0.12\end{array}$ & $\begin{array}{c}0.25 \\
\pm 0.17\end{array}$ & $\ldots$ & 11.76 & $3410.39 \pm 76.12$ & $3386.60 \pm 75.06$ & $23.80 \pm 6.84$ & $33 \pm 10$ & $0.3 \pm 0.0$ & 1 \\
\hline 050801 & 1.56 & ISMII & ISMII & $\begin{array}{c}2.78 \\
\pm 0.28\end{array}$ & $\begin{array}{c}0.22 \\
\pm 0.15\end{array}$ & $2.7 \mathrm{E}-05$ & $0.52 \pm 0.11$ & $124.89 \pm 17.39$ & $81.83 \pm 20.10$ & $43.06 \pm 17.65$ & $1 \pm 1$ & $0.4 \pm 0.1$ & 1 \\
\hline 051109A & 2.35 & $\begin{array}{l}\text { windI- } \\
\text { windII }\end{array}$ & $\begin{array}{l}\text { windI- } \\
\text { windII }\end{array}$ & $2.4 \pm 0.1$ & $\begin{array}{c}0.22 \\
\pm 0.06\end{array}$ & $\ldots$ & $9.15 \pm 6.83$ & $\begin{array}{c}14675.86 \pm \\
763.28\end{array}$ & $\begin{array}{c}14060.42 \pm \\
730.61\end{array}$ & $615.44 \pm 38.42$ & $1 \pm 1$ & $0.1 \pm 0.1$ & 1 \\
\hline 060206 & 4.05 & $\begin{array}{l}\text { windI- } \\
\text { windII }\end{array}$ & windI & $2.46 \pm 0.1$ & $\begin{array}{l}0.41 \\
\pm 0.06\end{array}$ & $\ldots$ & $4.78 \pm 20.79$ & $386.76 \pm 93.02$ & $344.28 \pm 83.18$ & $42.47 \pm 15.11$ & $10 \pm 44$ & $1 \pm 5$ & 1 \\
\hline 060714 & 2.71 & $\begin{array}{l}\text { windI- } \\
\text { windII }\end{array}$ & $\begin{array}{l}\text { windI- } \\
\text { windII }\end{array}$ & $\begin{array}{l}1.89 \\
\pm 0.07\end{array}$ & $\begin{array}{l}0.19 \\
\pm 0.05\end{array}$ & $\ldots$ & $18.22 \pm 2.53$ & $250.46 \pm 248.11$ & $239.83 \pm 237.24$ & $10.62 \pm 11.25$ & $63 \pm 67$ & $7 \pm 7$ & 1 \\
\hline 060729 & 0.54 & $\begin{array}{l}\text { windI- } \\
\text { windII }\end{array}$ & $\begin{array}{l}\text { windI- } \\
\text { windII }\end{array}$ & $\begin{array}{c}2.04 \\
\pm 0.08\end{array}$ & $\begin{array}{l}0.06 \\
\pm 0.01\end{array}$ & $\cdots$ & $0.75 \pm 0.07$ & $2312.14 \pm 33.54$ & $2304.60 \pm 33.43$ & $7.54 \pm 0.12$ & $9 \pm 1$ & $\begin{array}{l}0.03 \\
\pm 0.01\end{array}$ & 1 \\
\hline 070411 & 2.95 & $\begin{array}{l}\text { windI- } \\
\text { windII }\end{array}$ & $\begin{array}{l}\text { windI- } \\
\text { windII }\end{array}$ & $\begin{array}{c}2.48 \\
\pm 0.02\end{array}$ & $\begin{array}{c}0.86 \\
\pm 0.09\end{array}$ & $\ldots$ & $17.06 \pm 1.84$ & $\begin{array}{c}24456.57 \pm \\
256.42\end{array}$ & $\begin{array}{c}14460.59 \pm \\
794.78\end{array}$ & $9995.98 \pm 782.91$ & $0.2 \pm 0.1$ & $0.1 \pm 0.1$ & 1 \\
\hline 070518 & 1.16 & $\begin{array}{l}\text { windI- } \\
\text { windII }\end{array}$ & $\begin{array}{l}\text { windI- } \\
\text { windII }\end{array}$ & $2.4 \pm 0.2$ & $\begin{array}{c}0.24 \\
\pm 0.08\end{array}$ & $\ldots$ & $0.27 \pm 0.13$ & $917.47 \pm 22.99$ & $911.31 \pm 22.81$ & $6.16 \pm 0.52$ & $4 \pm 2$ & $\begin{array}{c}0.03 \\
\pm 0.01\end{array}$ & 1 \\
\hline 080710 & 0.85 & ISMII & ISMII & 2.78 & 0.32 & $9.6 \mathrm{E}-06$ & $1.34 \pm 0.32$ & $424.26 \pm 40.68$ & $393.99 \pm 37.78$ & $30.27 \pm 2.90$ & $4 \pm 1$ & $0.3 \pm 0.1$ & 1 \\
\hline 090102 & 1.55 & $\begin{array}{l}\text { windI- } \\
\text { windII }\end{array}$ & $\begin{array}{l}\text { windI- } \\
\text { windII }\end{array}$ & $\begin{array}{l}1.58 \\
\pm 0.22\end{array}$ & $\begin{array}{c}0.33 \\
\pm 0.05\end{array}$ & $\ldots$ & $22.74 \pm 2.12$ & $\begin{array}{c}1750.25 \pm \\
1149.59\end{array}$ & $\begin{array}{c}1490.68 \pm \\
1009.33\end{array}$ & $259.57 \pm 141.45$ & $8 \pm 4$ & $1 \pm 1$ & 1 \\
\hline 090426 & 2.61 & $\begin{array}{l}\text { windI- } \\
\text { windII }\end{array}$ & windI & $2.06 \pm 0.3$ & $\begin{array}{c}0.13 \\
\pm 0.02\end{array}$ & $\ldots$ & $0.83 \pm 0.28$ & $2.77 \pm 3.33$ & $1.80 \pm 2.17$ & $0.97 \pm 1.17$ & $46 \pm 58$ & $23 \pm 29$ & 1 \\
\hline 100418A & 0.62 & ISMII & ISMII & $\begin{array}{c}2.96 \\
\pm 0.18\end{array}$ & $\begin{array}{l}0.05 \\
\pm 0.02\end{array}$ & 2.9E-06 & $0.14 \pm 0.02$ & $1754.24 \pm 403.22$ & $1754.06 \pm 403.18$ & $0.18 \pm 0.05$ & $43 \pm 14$ & $\begin{array}{l}0.01 \\
\pm 0.01\end{array}$ & 1 \\
\hline $101024 \mathrm{~A}$ & $\ldots$ & ISMII & ISMII & $\begin{array}{c}2.64 \\
\pm 0.26\end{array}$ & $\begin{array}{l}0.06 \\
\pm 0.11\end{array}$ & $\ldots$ & $\ldots$ & $\ldots$ & $\ldots$ & $\ldots$ & $\ldots$ & $\ldots$ & 1 \\
\hline 050820A & 2.61 & ISMI-ISMII & $\begin{array}{l}\text { ISMI- } \\
\text { ISMII }\end{array}$ & $1.78 \pm 0.1$ & $\ldots$ & $\ldots$ & $\begin{array}{l}114.67 \\
\pm 33.59\end{array}$ & $\ldots$ & $\ldots$ & $4117.25 \pm 2462.50$ & $3 \pm 2$ & $\ldots$ & 2 \\
\hline 050922C & 2.20 & ISMII & ISMI & $\begin{array}{c}2.06 \\
\pm 0.05\end{array}$ & $\ldots$ & $\ldots$ & $9.93 \pm 1.06$ & $\ldots$ & $\ldots$ & $118.25 \pm 17.29$ & $8 \pm 1$ & $\ldots$ & 2 \\
\hline 060111B & $\ldots$ & ISMI & ISMI & $\begin{array}{l}1.57 \\
\pm 0.03\end{array}$ & $\ldots$ & $\ldots$ & $11.00 \pm 5.00$ & $\ldots$ & $\ldots$ & $\ldots$ & $\ldots$ & $\cdots$ & 2 \\
\hline 081008 & 1.97 & ISMII & ISMI & $\begin{aligned} & 1.96 \\
\pm & 0.18\end{aligned}$ & $\cdots$ & $\ldots$ & $9.30 \pm 1.91$ & $\ldots$ & $\cdots$ & $253.98 \pm 93.28$ & $4 \pm 3$ & $\ldots$ & 2 \\
\hline 081203A & 2.10 & ISMI-ISMII & $\begin{array}{l}\text { ISMI- } \\
\text { ISMII }\end{array}$ & $2.2 \pm$ & $\cdots$ & $\ldots$ & $34.71 \pm 17.12$ & $\cdots$ & $\ldots$ & $594.07 \pm 14.99$ & $6 \pm 3$ & $\ldots$ & 2 \\
\hline 090618 & 0.54 & ISMII & ISMI & $\begin{array}{l}1.92 \\
\pm 0.02\end{array}$ & $\cdots$ & $\cdots$ & $25.30 \pm 0.00$ & $\cdots$ & $\ldots$ & $89.10 \pm 14.81$ & $22 \pm 4$ & $\cdots$ & 2 \\
\hline 091127 & 0.49 & ISMII & ISMI & $1.36 \pm$ & $\ldots$ & $\ldots$ & $1.52 \pm 0.08$ & $\ldots$ & $\ldots$ & $263.04 \pm 22.94$ & $1 \pm 0.1$ & $\ldots$ & 2 \\
\hline $130427 \mathrm{~A}$ & 0.34 & ISMII & ISMII & $\begin{array}{c}2.38 \\
\pm 0.02\end{array}$ & $\ldots$ & $9.1 \mathrm{E}-06$ & 81.00 & $\ldots$ & $\ldots$ & $1665.96 \pm 62.14$ & $5 \pm 0.2$ & $\ldots$ & 2 \\
\hline 051028 & 3.70 & ISMI-SIMII & $\begin{array}{l}\text { ISMI- } \\
\text { ISMII }\end{array}$ & $1.9 \pm 0.1$ & $\ldots$ & $\ldots$ & $11.94 \pm 1.87$ & $\ldots$ & $\ldots$ & $2515.02 \pm 1557.52$ & $0.5 \pm 0.3$ & $\ldots$ & 4 \\
\hline 060418 & 1.49 & ISMII & $\begin{array}{l}\text { ISMI- } \\
\text { ISMII }\end{array}$ & $\begin{aligned} & 2.58 \\
\pm & 0.18\end{aligned}$ & $\cdots$ & $\cdots$ & $12.45 \pm 4.44$ & $\ldots$ & $\cdots$ & $386.85 \pm 42.95$ & $3 \pm 1$ & $\cdots$ & 4 \\
\hline 060512 & 0.44 & ISMI-SIMII & $\begin{array}{l}\text { ISMI- } \\
\text { ISMII }\end{array}$ & $\begin{array}{l}1.94 \\
\pm 0.06\end{array}$ & $\ldots$ & $\ldots$ & $0.02 \pm 0.01$ & $\ldots$ & $\ldots$ & $5.70 \pm 0.12$ & $0.3 \pm 0.2$ & $\ldots$ & 4 \\
\hline 060904B & 0.70 & ISMI-SIMII & $\begin{array}{l}\text { ISMI- } \\
\text { ISMII }\end{array}$ & $\begin{array}{l}3.25 \\
\pm 0.17\end{array}$ & $\cdots$ & $\cdots$ & $0.79 \pm 0.56$ & $\cdots$ & $\ldots$ & $750.73 \pm 388.34$ & $0.1 \pm 0.1$ & $\cdots$ & 4 \\
\hline 060912A & 0.94 & ISMII & ISMII & $2.2 \pm 0.3$ & $\ldots$ & 8.7E-05 & $1.12 \pm 0.08$ & $\ldots$ & $\ldots$ & $46.23 \pm 1.16$ & $2 \pm 0.2$ & $\ldots$ & 4 \\
\hline 060927 & 5.46 & windII & $\begin{array}{l}\text { windI- } \\
\text { windII }\end{array}$ & $2.22 \pm 0.1$ & $\cdots$ & $\ldots$ & $10.37 \pm 3.03$ & $\cdots$ & $\cdots$ & $37039.49 \pm 2373.94$ & $\begin{array}{l}0.03 \\
\pm 0.01\end{array}$ & $\cdots$ & 4 \\
\hline 061007 & 1.26 & ISMII & ISMII & $2.04 \pm 0.1$ & $\ldots$ & 7.3E-05 & $99.81 \pm 7.22$ & $\ldots$ & $\ldots$ & $1085.73 \pm 18.15$ & $8 \pm 1$ & $\ldots$ & 4 \\
\hline
\end{tabular}


Table 6

(Continued)

\begin{tabular}{|c|c|c|c|c|c|c|c|c|c|c|c|c|c|}
\hline GRB & $z$ & Optical $^{\mathrm{a}}$ & X-ray ${ }^{\mathrm{a}}$ & $p$ & $q$ & $\epsilon_{B}^{\mathrm{b}}$ & $E_{\gamma, \text { iso }}{ }^{\mathrm{c}}$ & $E_{\mathrm{K}, \text { end }}{ }^{\mathrm{c}}$ & $E_{\mathrm{K}, \text { in }}{ }^{\mathrm{c}}$ & $E_{\mathrm{K}, \mathrm{dec}}{ }^{\mathrm{c}}$ & $\eta_{\gamma, \mathrm{dec}}{ }^{\mathrm{d}}$ & $\eta_{\gamma, \text { end }}{ }^{\mathrm{d}}$ & Type $^{\mathrm{e}}$ \\
\hline 061126 & 1.16 & ISMII & ISMII & $\begin{array}{c}2.64 \\
\pm 0.18\end{array}$ & $\ldots$ & $1.0 \mathrm{E}-05$ & $13.26 \pm 1.90$ & $\ldots$ & $\ldots$ & $1282.64 \pm 177.08$ & $1 \pm 0.1$ & $\ldots$ & 4 \\
\hline 070318 & 0.84 & ISMII-ISMII & $\begin{array}{l}\text { ISMI- } \\
\text { ISMII }\end{array}$ & $\begin{array}{c}2.74 \\
\pm 0.02\end{array}$ & $\ldots$ & $\ldots$ & $1.39 \pm 0.35$ & $\ldots$ & $\ldots$ & $277.28 \pm 22.56$ & $0.5 \pm 0.1$ & $\ldots$ & 4 \\
\hline 071025 & 1.55 & ISMII & ISMII & $\begin{array}{l}3.07 \\
\pm 0.13\end{array}$ & $\ldots$ & $9.2 \mathrm{E}-06$ & $85.42 \pm 12.42$ & $\ldots$ & $\ldots$ & $1394.60 \pm 851.47$ & $6 \pm 4$ & $\ldots$ & 4 \\
\hline 071031 & 2.69 & ISMI & ISMI & $\begin{array}{c}2.01 \\
\pm 0.27\end{array}$ & $\ldots$ & $\ldots$ & $4.73 \pm 9.26$ & $\ldots$ & $\ldots$ & $68.53 \pm 38.27$ & $6 \pm 5$ & $\ldots$ & 4 \\
\hline 080319C & 1.95 & windI-II & $\begin{array}{l}\text { windI- } \\
\text { windII }\end{array}$ & $2.22 \pm 0.2$ & $\ldots$ & $\ldots$ & $23.54 \pm 1.47$ & $\ldots$ & $\ldots$ & $12314.17 \pm 3183.23$ & $0.2 \pm 0.1$ & $\ldots$ & 4 \\
\hline 080413A & 2.43 & windII & $\begin{array}{l}\text { windI- } \\
\text { windII }\end{array}$ & $2.3 \pm 0.48$ & $\ldots$ & $\ldots$ & $14.09 \pm 7.30$ & $\ldots$ & $\ldots$ & $249.98 \pm 71.42$ & $5 \pm 3$ & $\ldots$ & 4 \\
\hline 080603A & 1.68 & ISMI & ISMI & $\begin{aligned} & 2.96 \\
\pm & 0.08\end{aligned}$ & $\ldots$ & $\ldots$ & $2.20 \pm 0.80$ & $\ldots$ & $\ldots$ & $3577.17 \pm 272.70$ & $0.1 \pm 0.1$ & $\ldots$ & 4 \\
\hline 080804 & 2.20 & $\begin{array}{l}\text { windI- } \\
\text { windII }\end{array}$ & $\begin{array}{l}\text { windI- } \\
\text { windII }\end{array}$ & 1.86 & $\ldots$ & $\ldots$ & $24.61 \pm 4.79$ & $\ldots$ & $\ldots$ & $143.09 \pm 16.57$ & $15 \pm 3$ & $\ldots$ & 4 \\
\hline 080913 & 6.70 & ISMI-SIMII & $\begin{array}{l}\text { ISMI- } \\
\text { ISMII }\end{array}$ & $\begin{array}{l}2.79 \\
\pm 0.27\end{array}$ & $\ldots$ & $\ldots$ & $8.44 \pm 1.55$ & $\ldots$ & $\ldots$ & $354.60 \pm 174.38$ & $2 \pm 1$ & $\ldots$ & 4 \\
\hline 080928 & 1.69 & ISMII & ISMII & $\begin{array}{c}2.44 \\
\pm 0.04\end{array}$ & $\ldots$ & $5.7 \mathrm{E}-05$ & $6.30 \pm 0.75$ & $\ldots$ & $\ldots$ & $548.00 \pm 26.66$ & $1 \pm 0.1$ & $\ldots$ & 4 \\
\hline 090323 & 3.57 & windII & windII & $\begin{array}{l}2.65 \\
\pm 0.13\end{array}$ & $\ldots$ & $9.5 \mathrm{E}-04$ & $\begin{array}{l}372.38 \\
\pm 16.86\end{array}$ & $\ldots$ & $\ldots$ & $\begin{array}{c}209526.73 \pm \\
18842.90\end{array}$ & $0.2 \pm 0.1$ & $\ldots$ & 4 \\
\hline 090328 & 0.74 & ISMII & ISMII & $\begin{array}{l}3.19 \\
\pm 0.21\end{array}$ & $\ldots$ & $3.4 \mathrm{E}-06$ & 19.03 & $\ldots$ & $\ldots$ & $3861.76 \pm 2039.23$ & $0.5 \pm 0.3$ & $\ldots$ & 4 \\
\hline 090926A & 2.11 & ISMII & ISMII & $\begin{aligned} & 2.29 \\
\pm & 0.19\end{aligned}$ & $\ldots$ & $\ldots$ & $185.13 \pm 9.10$ & $\ldots$ & $\ldots$ & $1859.10 \pm 111.85$ & $9 \pm 1$ & $\ldots$ & 4 \\
\hline $100901 \mathrm{~A}$ & 1.41 & windII & $\begin{array}{l}\text { windI- } \\
\text { windII }\end{array}$ & $2.1 \pm 0.1$ & $\ldots$ & $\ldots$ & $2.95 \pm 0.63$ & $\ldots$ & $\ldots$ & $27847.74 \pm 871.45$ & $\begin{array}{l}0.01 \\
\pm 0.01\end{array}$ & $\ldots$ & 4 \\
\hline $120326 \mathrm{~A}$ & 1.80 & windII & windII & $\begin{array}{l}2.51 \\
\pm 0.09\end{array}$ & $\ldots$ & $5.2 \mathrm{E}-04$ & $3.18 \pm 0.40$ & $\ldots$ & $\ldots$ & $41757.87 \pm 1069.46$ & $\begin{array}{l}0.01 \\
\pm 0.01\end{array}$ & $\ldots$ & 4 \\
\hline \multicolumn{14}{|l|}{ Grade II } \\
\hline 051111 & 1.55 & windII & $\begin{array}{l}\text { windI- } \\
\text { windII }\end{array}$ & $\begin{array}{c}2.52 \\
\pm 0.14\end{array}$ & $\ldots$ & $\ldots$ & $10.79 \pm 3.07$ & $\ldots$ & $\ldots$ & $3793.13 \pm 273.16$ & $0.3 \pm 0.1$ & $\ldots$ & 4 \\
\hline 090313 & 3.38 & windII & $\begin{array}{l}\text { windI- } \\
\text { windII }\end{array}$ & $\begin{array}{c}2.54 \\
\pm 0.72\end{array}$ & $\ldots$ & $5.0 \mathrm{E}-06$ & $13.02 \pm 2.94$ & $\ldots$ & $\ldots$ & $\begin{array}{c}173028.58 \pm \\
189747.00\end{array}$ & $\begin{array}{c}0.01 \\
\pm 0.01\end{array}$ & $\ldots$ & 4 \\
\hline
\end{tabular}

Notes.

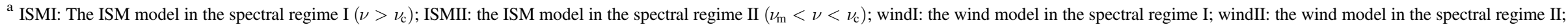

ISMI-ISMII: the ISM model in the gray zone (between $\nu>\nu_{\mathrm{c}}$ and $\nu_{\mathrm{m}}<\nu<\nu_{\mathrm{c}}$ ); windI-windII: the wind model in the gray zone.

${ }^{\mathrm{b}}$ Upper limit, calculated with the X-ray data in the spectral regime II $\left(\nu_{\mathrm{m}}<\nu<\nu_{\mathrm{c}}\right)$.

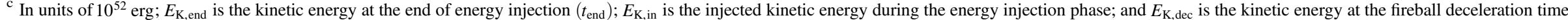

$\left(t_{\mathrm{dec}}\right)$.

${ }^{\mathrm{d}}$ In units of $\% ; \eta_{\gamma, \text { dec }}$ is the radiative efficiency calculated using $E_{\mathrm{K}, \mathrm{dec}} ; \eta_{\gamma, \text { dec }}$ is the radiative efficiency calculated using $E_{\mathrm{K} \text {,end }}$.

${ }^{\mathrm{e}}$ 1: Energy injection break; 2: jet break; 3: jet break with energy injection; 4: SPL decay. 

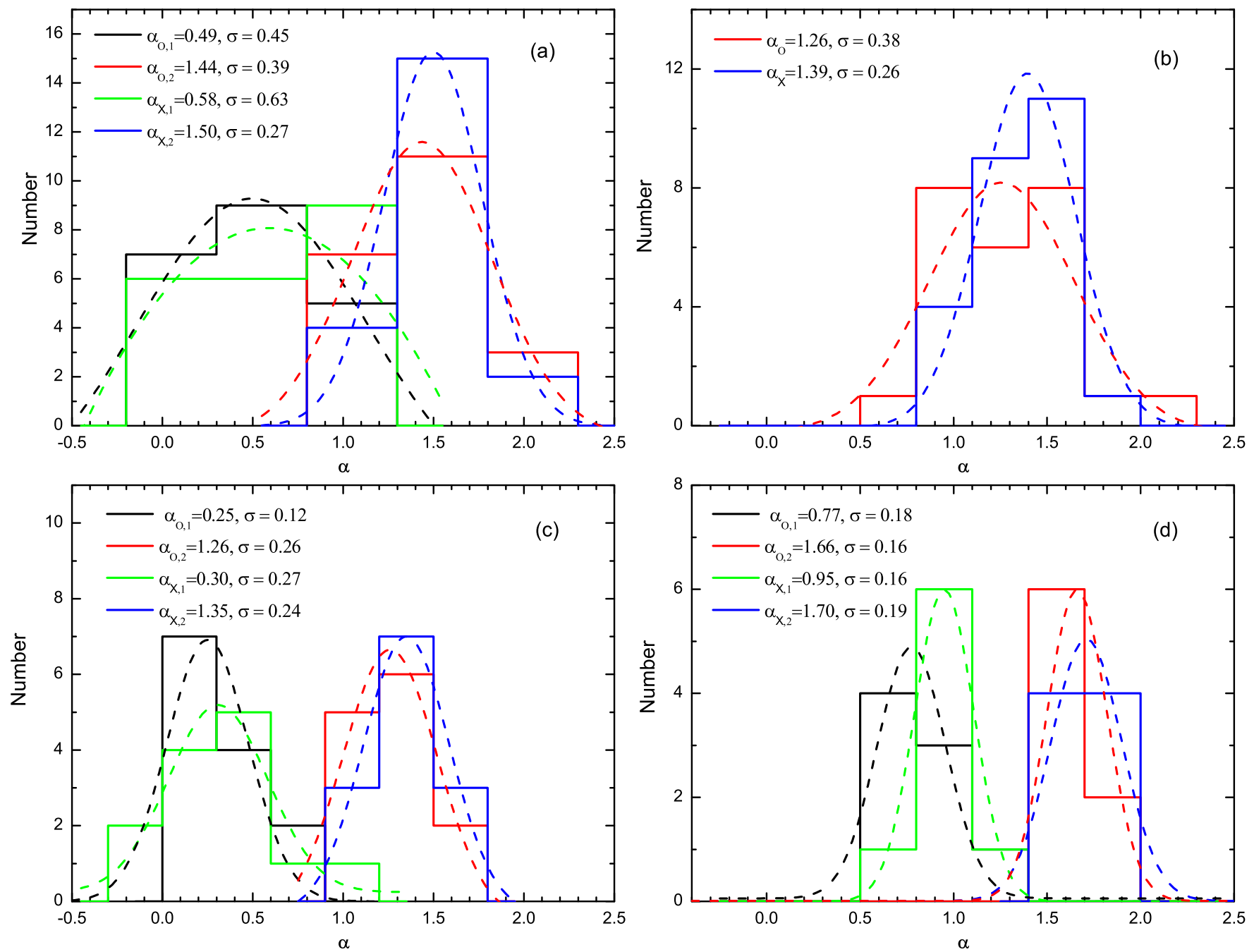

Figure 8. Distributions of the temporal decay index $\alpha$ for various subsamples in the Gold sample and their best Gaussian fits (dashed curves): (a) all of the BPL GRBs in the Gold sample, with best fits $\alpha_{\mathrm{O}, 1}=0.49 \pm 0.45, \alpha_{\mathrm{O}, 2}=1.44 \pm 0.39, \alpha_{\mathrm{X}, 1}=0.58 \pm 0.63$, and $\alpha_{\mathrm{X}, 2}=1.50 \pm 0.27$; (b) all of the SPL GRBs in the Gold sample, with best fits $\alpha_{\mathrm{O}}=1.26 \pm 0.38$ and $\alpha_{\mathrm{X}}=1.39 \pm 0.26$; (c) the energy injection sample, with $\alpha_{\mathrm{O}, 1}=0.25 \pm 0.12, \alpha_{\mathrm{O}, 2}=1.26 \pm 0.26$, $\alpha_{\mathrm{X}, 1}=0.30 \pm 0.27$, and $\alpha_{\mathrm{X}, 2}=1.35 \pm 0.24 ; \quad$ (d) the jet break sample, with $\alpha_{0,1}=0.77 \pm 0.18, \quad \alpha_{0,2}=1.66 \pm 0.16, \quad \alpha_{\mathrm{X}, 1}=0.95 \pm 0.16, \quad$ and $\alpha_{\mathrm{X}, 2}=1.70 \pm 0.19$.

\subsection{Radiative Efficiency $\eta_{\gamma}$}

The GRB radiative efficiency, defined as (Lloyd-Ronning \& Zhang 2004)

$$
\eta_{\gamma}=\frac{E_{\gamma, \text { iso }}}{E_{\gamma, \text { iso }}+E_{\mathrm{K}, \text { iso }}}
$$

is an essential parameter to probe how efficiently a burst converts its global energy into prompt $\gamma$-ray emission. As mentioned above, if there is a continuous energy injection, the kinetic energy of the afterglow $E_{\mathrm{K} \text {,iso }}$ takes different values if one chooses different epochs. In principle, $\eta_{\gamma}$ can be defined for two different epochs, $t_{\mathrm{dec}}$ and $t_{\mathrm{b}}$, which have different physical meanings (see a discussion in Zhang et al. 2007a).

Figure 18 shows the radiative efficiencies calculated at $t_{\mathrm{dec}}$ and $t_{\mathrm{b}}$ as a function of $E_{\gamma, \text { iso }}$ along with their histograms. No significant correlation between $\eta_{\gamma}$ and $E_{\gamma, \text { iso }}$ is found. The fireball internal shock model predicts a relatively small efficiency of a few percent (e.g., Kumar 1999; Panaitescu et al. 1999; Maxham \& Zhang 2009; Gao \& Mészáros 2015).
Previous constraints on GRB radiative efficiencies give relatively large values, as large as above $90 \%$ (e.g., LloydRonning \& Zhang 2004; Zhang et al. 2007a; Racusin et al. 2011) for some GRBs. This challenges the internal shock models and favors alternative prompt emission models, such as dissipation of magnetic fields (Zhang \& Yan 2011) or photospheric emission (Lazzati et al. 2013).

The derived efficiencies can be fit with rough log-normal distributions (Figures 18(b), (d), (f)). For the entire sample, one has $\log \left(\eta_{\gamma, \mathrm{dec}} / \%\right)=0.75 \pm 0.86$. For the subsample of GRBs without energy injection, the radiative efficiency is lower, with $\log \left(\eta_{\gamma, \mathrm{dec}} / \%\right)=0.37 \pm 0.61$. For the subsample of GRBs with energy injection, the radiative efficiencies read as $\log \left(\eta_{\gamma, \mathrm{dec}} / \%\right)=0.92 \pm 1.25$ for $t_{\mathrm{dec}}$, and $\log \left(\eta_{\gamma, \text { end }} / \%\right)=$ $-0.89 \pm 0.97$ for $t_{\mathrm{b}}$, respectively.

The derived efficiencies are somewhat smaller than the values derived in previous work (e.g., Zhang et al. 2007a). The main reason is the adoption of a smaller value of $\epsilon_{B} \sim 10^{-5}$, so the derived $E_{K \text {,iso }}$ are systematically larger. This greatly alleviates the low-efficiency problem of the internal shock 


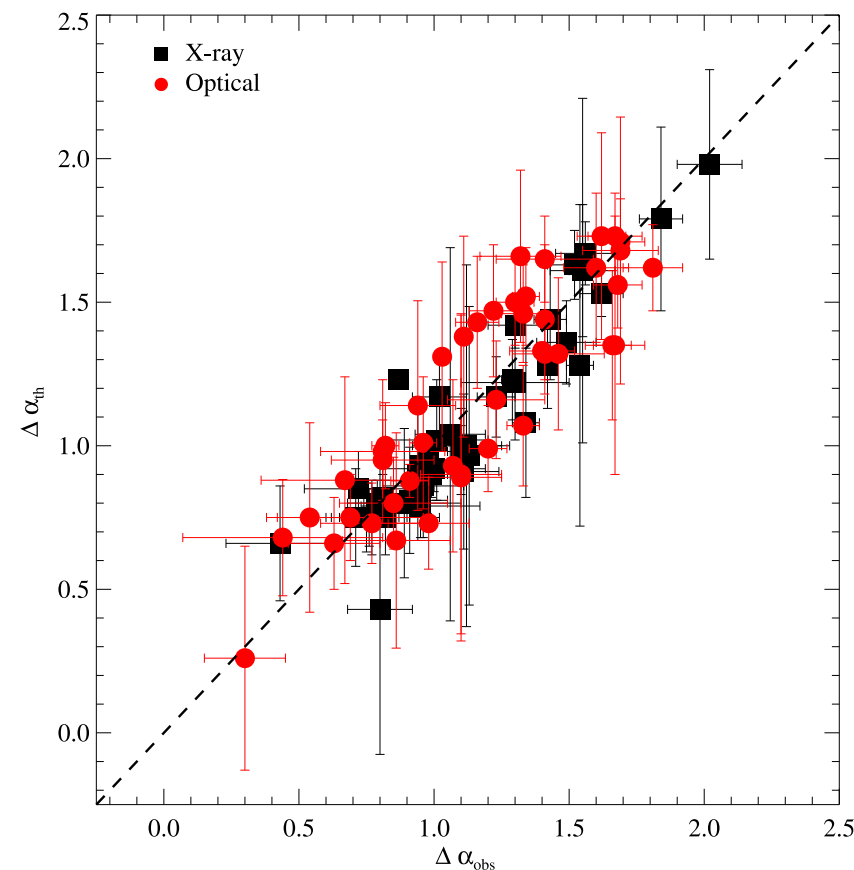

Figure 9. Observed temporal break change $\Delta \alpha=\alpha_{2}-\alpha_{1}$ compared against the theoretical value predicted from the closure relations. The square (black) and circle (red) data points correspond to the X-ray and optical bands, respectively.

models. Nonetheless, some GRBs still have tens of percent efficiency, which demands a contrived setup for the internal shock models (e.g., Beloborodov 2000; Kobayashi \& Sari 2001). If $t_{\mathrm{dec}}$ is adopted, which is more natural for most prompt emission models to calculate efficiency (see Zhang et al. 2007a for a detailed discussion), $\eta_{\gamma}$ is still typically too large for the internal shock model. This is, on the other hand, consistent with the suggestion that internal collision-induced magnetic reconnection and turbulence (ICMART) is the dominant process to power GRB prompt emission in the majority of GRBs, which can typically gives tens of percent radiative efficiency (Zhang \& Yan 2011; Deng et al. 2015). This conclusion is also consistent with independent studies of modeling the GRB prompt emission spectrum (Uhm \& Zhang 2014c) and quasithermal photosphere emission component (Gao \& Zhang 2015).

\subsection{Jet Opening Angle and Geometrically Corrected Gamma-ray Energy}

In the Gold sample, 8 of 45 GRBs show a jet break. These include five GRBs without energy injection and one more with energy injection. The ambient medium type of all six GRBs is ISM. Five out of these six GRBs have redshift information (Table 7).

Under the assumption of a conical jet, one can derive the jet opening angle based on observational data (Rhoads 1999; Sari et al. 1999; Frail et al. 2001):

$$
\begin{aligned}
\theta_{j}= & 0.070 \mathrm{rad}\left(\frac{t_{\mathrm{b}}}{1 \text { day }}\right)^{3 / 8}\left(\frac{1+z}{2}\right)^{-3 / 8} \\
& \times\left(\frac{E_{\mathrm{K}, \text { iso }}}{10^{53} \mathrm{erg}}\right)^{-1 / 8}\left(\frac{n}{0.1 \mathrm{~cm}^{-3}}\right)^{1 / 8} .
\end{aligned}
$$

We then calculate the geometrically corrected $\gamma$-ray energy

$$
E_{\gamma}=\left(1-\cos \theta_{j}\right) E_{\gamma, \text { iso }}
$$

and kinetic energy

$$
E_{\mathrm{K}}=\left(1-\cos \theta_{j}\right) E_{\mathrm{K}, \text { iso }} .
$$

Here $E_{\mathrm{K} \text {,iso }}$ is taken as $E_{\mathrm{K} \text {,end }}$ for the energy injection sample. The medium density is taken as $n=1 \mathrm{~cm}^{-3}$. The results are presented in Table 7 and Figure 19. The best-fitting results give $\theta_{j}=(2.8 \pm 1.5), \quad \log \left(E_{\gamma} / \mathrm{erg}\right)=49.86 \pm 0.65$, and $\log \left(E_{\mathrm{K}} / \mathrm{erg}\right)=50.89 \pm 0.54$.

\section{CONCLUSIONS AND DISCUSSION}

The chromatic afterglow behavior observed in some GRBs has raised concern regarding whether the external forward shock models are still adequate to interpret the broadband afterglows of GRBs and whether alternative ideas, such as a long-lasting engine-driven afterglow, are needed to account for the data. In order to answer in this paper how bad or good the external shock models are, we systematically studied 85 Swift GRBs up to 2014 March, which all have high-quality X-ray and optical light curves and spectral data to allow us to study the compliance of the data to the external forward shock models. The results of this study are summarized in the following.

Based on how well the data abide by the external forward shock afterglow models, we categorized GRBs into five grades and three samples.

1. A Gold sample (Grade I and II) includes 45/85 GRBs. These GRBs are fully consistent with the theoretical predictions of the external shock models, including having an acceptable achromatic break and fulfilling various closure relations between the temporal decay indices $\alpha$ and spectral indices $\beta$.

2. A Silver sample (Grade III and IV) includes 37/85 GRBs. These GRBs are also consistent with having an acceptable achromatic break, even though one or more afterglow segments do not comply with the closure relations. These GRBs are potentially interpretable within the framework of external shock models.

3. A Bad sample (Grade V) only includes 3/85 GRBs. These GRBs show direct evidence of chromatic behaviors, which cannot be accounted for within singlecomponent external shock models.

The bottom line of this study is to address how bad or good the external shock models are. Our results show that external shock models work very well for at least $\sim 53 \%$ of GRBs (our Gold sample). These GRBs can be interpreted within the simplest afterglow models. If more advanced modeling invoking other factors (e.g., structured jet or long-lasting reverse shock) is carried out, up to $\sim 96 \%$ of GRBs (including the Silver sample) may be accounted for within the external shock models. Only less than $4 \%$ GRBs truly violate the basic expectations of the external shock models and demand another emission component (e.g., central engine afterglow) to account for emission in at least one band (e.g., the $\mathrm{X}$-ray band).

Several caveats deserve mentioning. First, we only focused on the main afterglow components (SPL, BPL, or TPL) of the X-ray and optical light curves. In some GRBs, there 

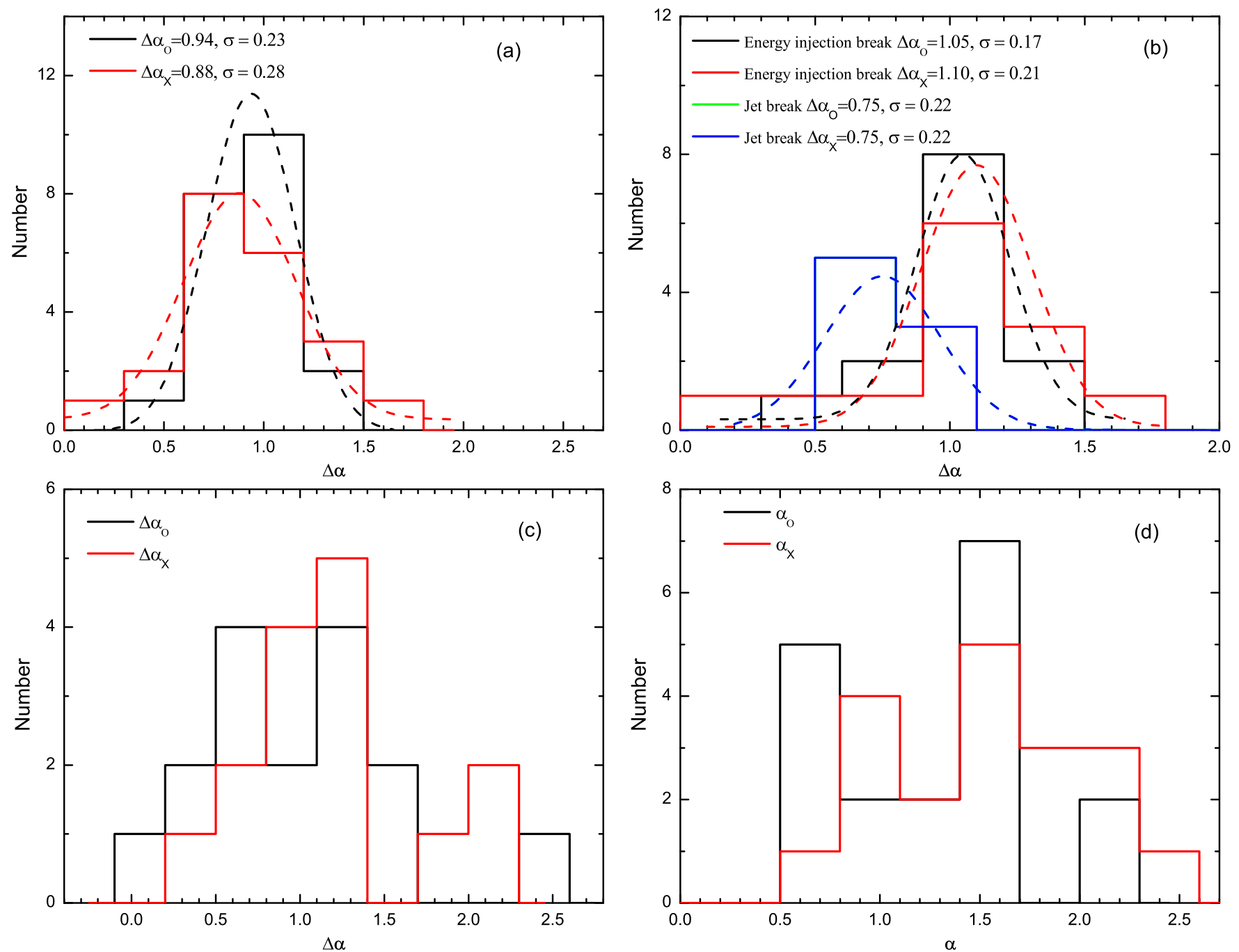

Figure 10. Distributions of the temporal break change $\Delta \alpha=\alpha_{2}-\alpha_{1}$ for various subsamples in the Gold sample, and the $\Delta \alpha$ and $\alpha$ distributions of the Silver sample. The best Gaussian fits are marked with dashed curves: (a) the $\Delta \alpha$ distributions of the entire Gold sample, with $\Delta \alpha_{\mathrm{O}}=0.94 \pm 0.23$ and $\Delta \alpha_{\mathrm{X}}=0.88 \pm 0.28$; (b) the $\Delta \alpha$ distributions of different subsamples in the Gold sample, with $\Delta \alpha_{\mathrm{O}}=1.05 \pm 0.17$ and $\Delta \alpha_{\mathrm{X}}=1.10 \pm 0.21$ for the energy injection breaks, and $\Delta \alpha_{\mathrm{O}}=0.75 \pm 0.22$ and $\Delta \alpha_{\mathrm{X}}=0.75 \pm 0.22$ for the jet breaks; (c) and (d) the $\Delta \alpha$ distributions for the BPL and SPL GRBs in the Silver sample, respectively. The data are dispersed.

are additional components overlapping with these main components, such as the X-ray steep decay phase, X-ray flares, and optical rebrightening features, which are not included in the analysis. These features are usually chromatic and demand additional emission components to interpret the data. The general conclusion that a lot of GRBs have extended central engine activities (Zhang et al. 2006) remains valid. The true duration of the GRB central engine activities may be much longer than what is measured by the GRB duration $T_{90}$ (Zhang et al. 2014). Second, we adopted a relatively "loose" criterion $\left(\chi_{\text {total }}^{2} \leqslant 3\right)$ to define "achromaticity" by requiring the X-ray and optical light curves to have the same break time. Searching for break times independently in the two bands often results in somewhat different break times, but many GRBs can be made consistent with being achromatic. The relatively large $\chi_{\text {total }}^{2} \sim 3$ in some GRBs is mostly caused by additional features (small flares and fluctuations that we do not care about) in the otherwise (broken) power-law light curves. We therefore believe that our approach is appropriate to address the question of how bad the models are. On the other hand, if in the future high-quality data indeed show slight chromatic behaviors with high confidence, one should take cautiously the fraction numbers presented in this paper and consider how such slight chromatic behaviors may impact the models. Finally, we only studied 85 GRBs that have both bright X-ray and bright optical emission data to allow us to perform the test. There are more GRBs detected by Swift ( $\sim 900$ with X-ray light curves and $\sim 260$ with optical light curves). Because of complicated sample selection effects, we do not guarantee that the fractions of Gold, Silver, and Bad samples are reliable numbers for the entire GRB population. In any case, 85 GRBs represent a reasonably large sample, so our statistics are valid at least for the "bright" sample of GRBs.

With the Gold sample, we further performed a series of statistical analyses of various observational properties and model parameters. The following interesting conclusions can be drawn.

1. Temporal index $\alpha$ : The temporal indices $\alpha$ in different bands and different temporal segments satisfy the afterglow model predictions. On average, the X-ray light 

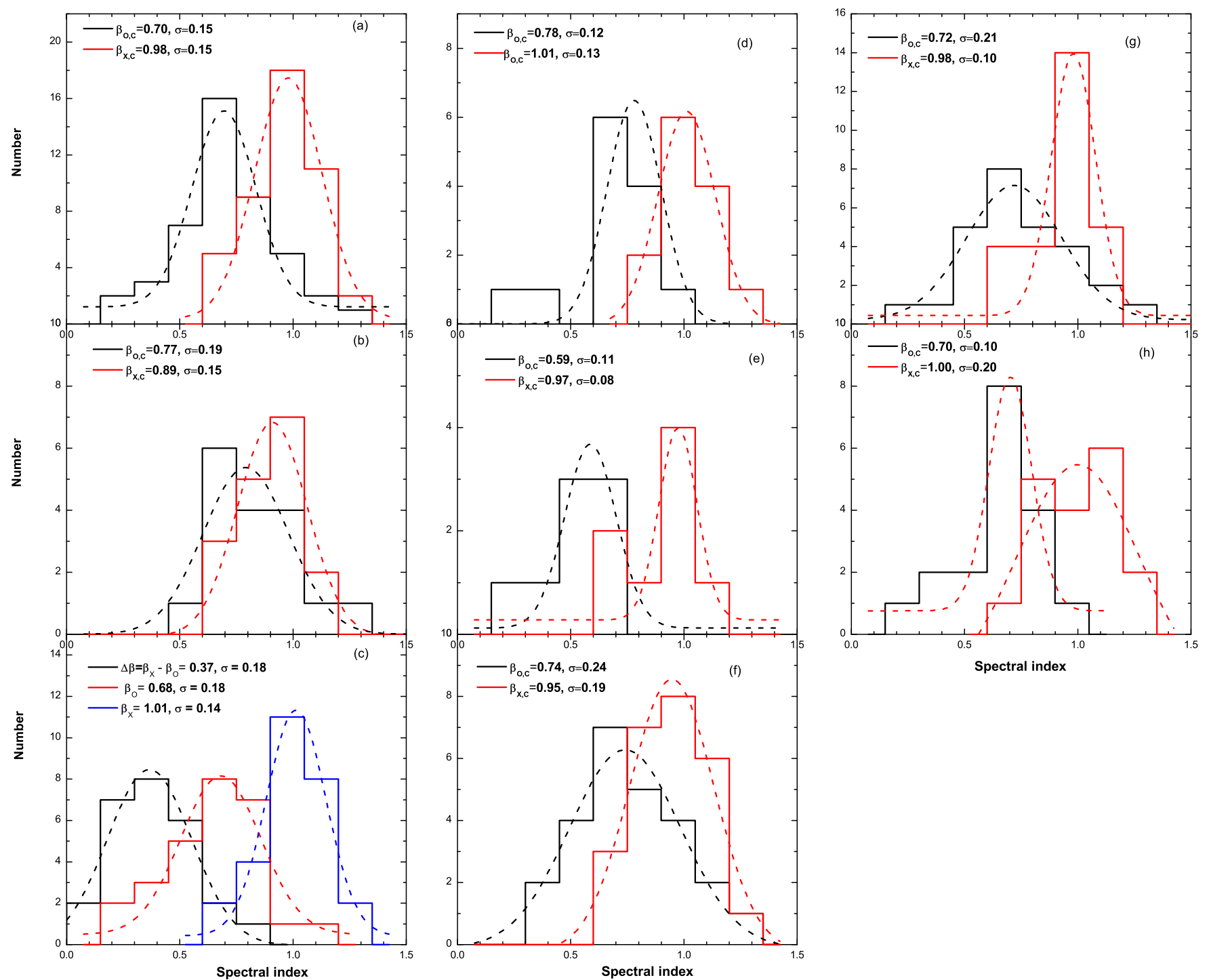

Figure 11. Distributions of the spectral indices $\beta$ of various subsamples in the Gold sample and their best Gaussian fits (dashed curves): (a) the distributions for the entire Gold sample, with $\beta_{\mathrm{O}}=0.70 \pm 0.15$ and $\beta_{\mathrm{X}}=0.98 \pm 0.15$; (b) the $\beta$ distributions of the GRBs with optical and X-ray bands constrained in the same spectral regime, with $\beta_{\mathrm{O}}=0.77 \pm 0.19$ and $\beta_{\mathrm{X}}=0.89 \pm 0.15$; (c) the $\beta$ distributions for the GRBs with optical and X-ray bands constrained in different spectral regimes, with $\beta_{\mathrm{O}}=0.68 \pm 0.18, \beta_{\mathrm{X}}=1.01 \pm 0.14$, and $\Delta \beta=\beta_{\mathrm{X}}-\beta_{\mathrm{O}}=0.37 \pm 0.18$, which is consistent with the theoretical value 0.5 as expected for a cooling break; (d) the $\beta$ distributions for energy injection, with $\beta_{\mathrm{O}}=0.78 \pm 0.12$ and $\beta_{\mathrm{X}}=1.01 \pm 0.13$; (e) the $\beta$ distributions for the jet break sample, with $\beta_{\mathrm{O}}=0.59 \pm 0.11$ and $\beta_{\mathrm{X}}=0.97 \pm 0.08$; (f) the $\beta$ distributions for the SPL decay sample, with $\beta_{\mathrm{O}}=0.74 \pm 0.24$ and $\beta_{\mathrm{X}}=0.95 \pm 0.19$; (g) the $\beta$ distributions for the GRBs with an ISM medium, with $\beta_{\mathrm{O}}=0.72 \pm 0.21$ and $\beta_{\mathrm{X}}=0.98 \pm 0.10$; (h) the $\beta$ distributions for the GRBs with a wind medium, with $\beta_{\mathrm{O}}=0.70 \pm 0.10$ and $\beta_{\mathrm{X}}=1.00 \pm 0.20$.

curves are steeper than optical. For BPL light curves, the degrees of the break, $\Delta \alpha$, are consistent with the theoretical predictions of the energy injection models or jet break models.

2. Spectral index $\beta$ : The spectral indices $\beta$ in the optical and $\mathrm{X}$-ray bands are $\beta_{\mathrm{O}}=0.70 \pm 0.15$ and $\beta_{\mathrm{X}}=$ $0.98 \pm 0.15$, respectively. Some (17/45) have X-ray and optical bands in the same spectral segment, while most $(28 / 45)$ have the two bands separated by $\nu_{\mathrm{c}}$ or in the gray zone. Statistically, $\Delta \beta=0.37 \pm 0.18$ is consistent with the theoretical value $0-0.5$, a range of $\Delta \beta$, including those expected in the gray zone (Uhm \& Zhang 2014a).

3. Electron spectral index $p$ : The typical value $p=2.33 \pm 0.48$ is very consistent with the theoretical predictions for relativistic shocks. A wide range of $p$ values is observed, which is consistent with previous findings.

4. Break time $t_{\mathrm{b}}$ : The typical break time is found to be $\log \left(t_{\mathrm{b}} / \mathrm{ks}\right)=3.8 \pm 0.9$. The break time of the energy injection sample $\left(\log \left(t_{\mathrm{b}} / \mathrm{ks}\right)=3.96 \pm 1.9\right)$ is statistically earlier than that of the jet break sample $\left(\log \left(t_{\mathrm{b}} / \mathrm{ks}\right)=3.9 \pm 0.7\right)$.

5. Energy injection parameter $q$ : The central value is $q=0.22 \pm 0.11$, and the ISM and wind models are consistent with each other, with $q=0.20 \pm 0.12$ and $q=0.23 \pm 0.13$, respectively.

6. Magnetization parameter $\epsilon_{B}$ : The derived upper limits of $\epsilon_{B}$ suggest that the typical value of this parameter is low (say, $10^{-5}$ ), which is consistent with previous work 

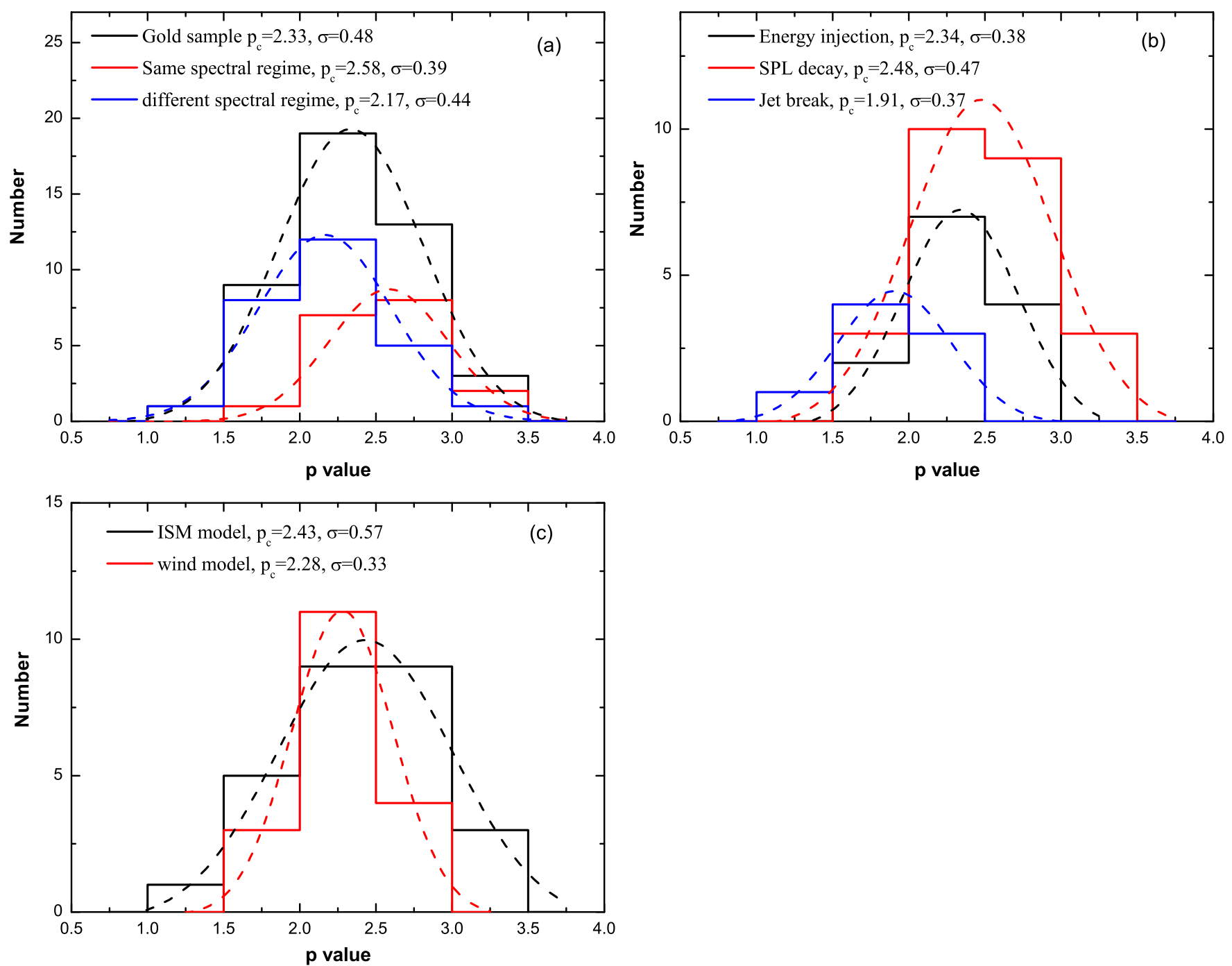

Figure 12. Distribution of the inferred electron spectral index $p$ from various subsamples in the Gold sample and their best Gaussian fits (dashed lines): (a) the entire Gold sample with $p=2.33 \pm 0.48$, and the subsamples with the optical and X-ray bands constrained in the same $(p=2.58 \pm 0.39)$ and different $(p=2.17 \pm 0.44)$ spectral regimes, respectively; (b) the subsamples with different light curve behaviors: energy injection $(p=2.34 \pm 0.38)$, jet break $(p=1.91 \pm 0.37)$, and SPL decay $(p=2.48 \pm 0.47)$; $(c)$ the subsamples with different medium types: $\operatorname{ISM}(p=2.43 \pm 0.57)$ and wind $(p=2.28 \pm 0.33)$.

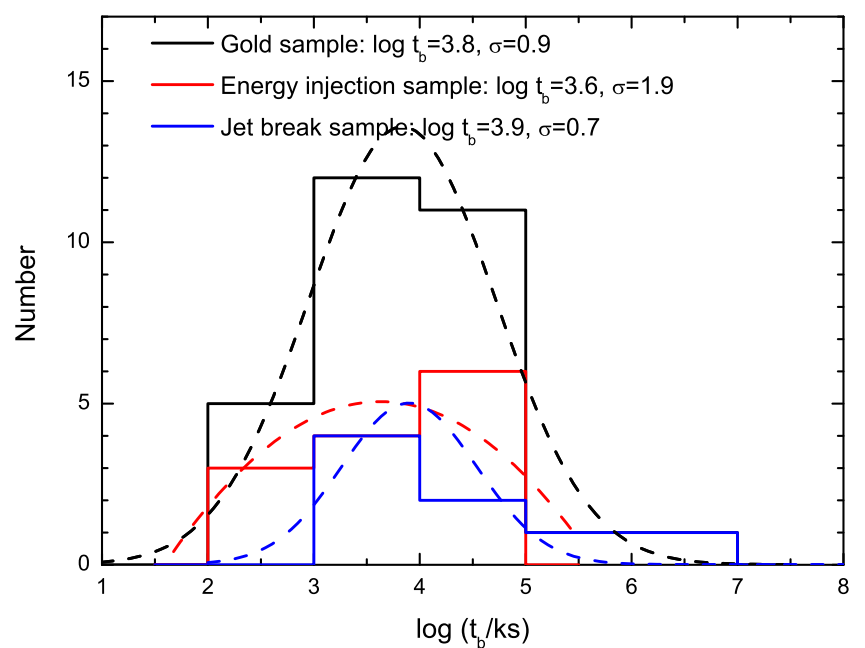

Figure 13. Distributions of the inferred $t_{\mathrm{b}}$ for the entire Gold sample, the energy injection sample, and the jet break sample. The dash lines are the best Gaussian fits: the entire Gold sample $\left(\log \left(t_{\mathrm{b}} / \mathrm{ks}\right)=(3.8 \pm 0.9)\right)$, the energy injection break sample $\left(\log \left(t_{\mathrm{b}} / \mathrm{ks}\right)=(3.6 \pm 1.9)\right)$, and the jet break sample $\left(\log \left(t_{\mathrm{b}} / \mathrm{ks}\right)=(3.9 \pm 0.7)\right)$.

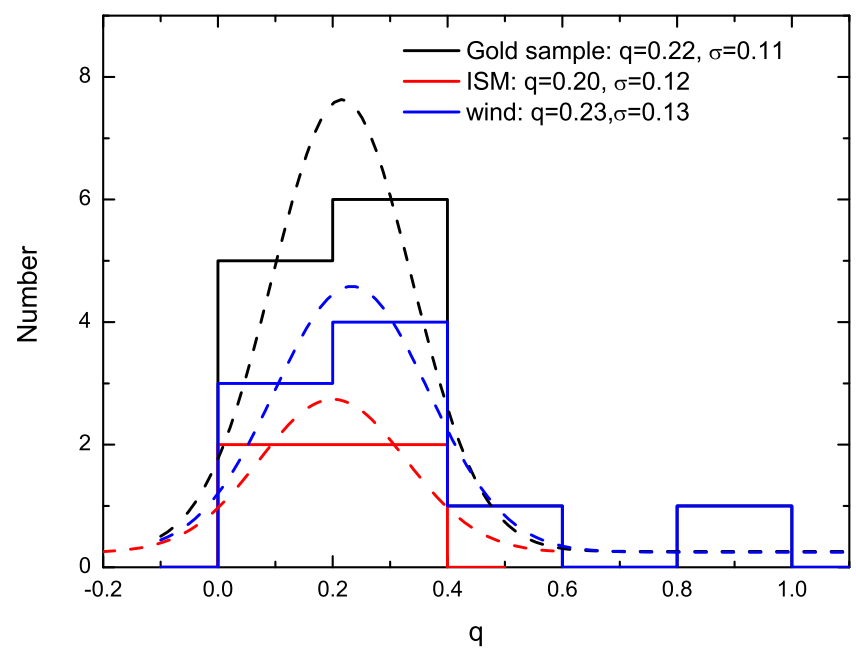

Figure 14. Distributions of the inferred energy injection parameter $q$ and their best Gaussian fits (dashed lines): the entire Gold sample $(q=0.22 \pm 0.11)$, the ISM sample $(q=0.20 \pm 0.12)$, and the wind sample $(q=0.23 \pm 0.13)$. 


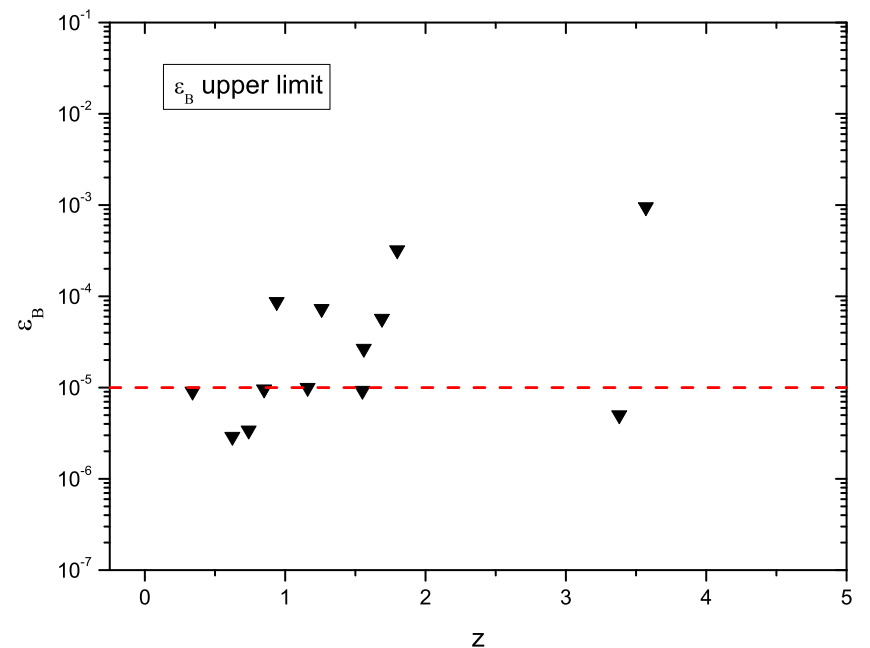

Figure 15. Derived upper limits of $\epsilon_{B}$ of GRBs that are in spectral regime II $\left(\nu_{\mathrm{m}}<\nu<\nu_{\mathrm{c}}\right)$. Other parameters are fixed as $\epsilon_{e}=0.1, n=1$, and $A_{*}=1$. The dashed line denotes $\epsilon_{B}=10^{-5}$. Most GRBs are consistent with this value, with a few having upper limits even below this value.

(Kumar \& Barniol Duran 2009, 2010; Barniol Duran 2014; Santana et al. 2014).

7. Energetics: The typical isotropic $\gamma$-ray energy is $\log \left(E_{\gamma, \text { iso }} / \mathrm{erg}\right)=53.15 \pm 0.69$. For the energy injection case, the typical isotropic kinetic energy in the blast wave is $\log \left(E_{\mathrm{K}, \mathrm{dec}} / \mathrm{erg}\right)=53.29 \pm 0.45$ at the deceleration time, and $\log \left(E_{\mathrm{K}, \text { end }} / \mathrm{erg}\right)=54.99 \pm 0.86$ when energy injection is over. For GRBs without energy injection, the typical blast wave kinetic energy is $\log \left(E_{\mathrm{K}, \mathrm{dec}} / \mathrm{erg}\right)=$ $54.66 \pm 1.18$. Clear correlations among various energy components are found.

8. Radiative efficiency $\eta_{\gamma}$ : With a small $\epsilon_{B} \sim 10^{-5}$ adopted, the derived radiative efficiency $\eta_{\gamma}$ is lower than previous studies. For the entire Gold sample, $\log \left(\eta_{\gamma, \mathrm{dec}} / \%\right)=$ $0.75 \pm 0.86$. Yet, the efficiency is still large for some GRBs, especially the ones with energy injection. For these GRBs, the efficiency measure at the deceleration time has $\log \left(\eta_{\gamma, \mathrm{dec}} / \%\right)=0.92 \pm 1.25$, which still challenges the internal shock model.

9. Jet opening angle $\theta_{j}$ : For the jet break sample, we derived the typical jet opening angle as $\theta_{j}=(2.5 \pm 1.5)$. The jet-corrected $\gamma$-ray energy and kinetic energy are $\log \left(E_{\gamma} /\right.$ erg $)=49.86 \pm 0.65$ and $\log \left(E_{\mathrm{K}} / \mathrm{erg}\right)=$ $50.89 \pm 0.54$, respectively.

We thank an anonymous referee for helpful suggestions and M. De Pasquale for a comment. This work is supported by the National Basic Research Program (973 Programme) of China (grant No. 2014CB845800), the National Natural Science Foundation of China (grants 11303005, U1331202), the Guangxi Science Foundation (2013GXNSFFA019001), the Strategic Priority Research Program "The Emergence of Cosmological Structures" (grant No. XDB09000000) B.Z. acknowledges NASA NNX14AF85G for support. H.G. acknowledges NASA NNX 13AH50G. D.A.K. acknowledges support by DFG grants Kl 766/16-1 and Kl 766/16-3."

\section{APPENDIX A EXPRESSIONS OF $E_{\mathrm{K}, \mathrm{ISO}}$ AND $\epsilon_{B}$}

In this appendix, we present expressions of $E_{\mathrm{K} \text {,iso }}$ and $\epsilon_{B}$ of the external forward shock afterglow models.

\section{A.1. The ISM Model}

For the $p>2$ case, the forward shock emission can be characterized as (Yost et al. 2003; Zhang et al. 2007a)

$$
\begin{aligned}
\nu_{\mathrm{m}}= & 3.3 \times 10^{12} \mathrm{~Hz}\left(\frac{p-2}{p-1}\right)^{2} \\
& \times(1+z)^{1 / 2} \epsilon_{B,-2}^{1 / 2} \epsilon_{e,-1}^{2} E_{\mathrm{K}, \text { iso }, 52}^{1 / 2} t_{d}^{-3 / 2}, \\
\nu_{\mathrm{c}}= & 6.3 \times 10^{15} \mathrm{~Hz}(1+z)^{-1 / 2} \\
& \times(1+Y)^{-2} \epsilon_{B,-2}^{-3 / 2} E_{\mathrm{K}, \mathrm{iso}, 52}^{-1 / 2} n^{-1} t_{d}^{-1 / 2}, \\
F_{\nu, \max }= & 1.6 \mathrm{mJy}(1+z) D_{28}^{-2} \epsilon_{B,-2}^{1 / 2} E_{\mathrm{K}, \text { iso }, 52} n^{1 / 2},
\end{aligned}
$$

where $E_{\mathrm{K}, \text { iso }, 52}$ is the isotropic kinetic energy (in units of $10^{52} \mathrm{erg}$ ) in the blast wave, $t_{d}$ is the time since trigger (in units of days), $n$ is the density of the constant ambient medium, $D_{L}=10^{28} \mathrm{~cm} D_{28}$ is the luminosity distance, and

$$
Y=\left[-1+\left(1+4 \eta_{1} \eta_{2} \epsilon_{e} / \epsilon_{B}\right)^{1 / 2}\right] / 2
$$

is the inverse Compton parameter, with $\eta_{1}=$ $\min \left[1,\left(\nu_{\mathrm{c}} / \nu_{\mathrm{m}}\right)^{(2-p) / 2}\right]\left(\right.$ Sari \& Esin 2001), and $\eta_{2} \leqslant 1$ is a correction factor introduced by the Klein-Nishina correction.

For $p>2$ and in the $\nu>\max \left(\nu_{\mathrm{m}}, \nu_{\mathrm{c}}\right)$ regime, one has (Zhang et al. 2007a)

$$
\begin{aligned}
\nu F_{\nu}\left(\nu=10^{18} \mathrm{~Hz}\right)= & F_{\nu, \max } \nu_{\mathrm{c}}^{1 / 2} \nu_{\mathrm{m}}^{(p-1) / 2} \nu_{\mathrm{X}^{(2-p) / 2}} \\
= & 5.2 \times 10^{-14} \mathrm{erg} \mathrm{s}^{-1} \mathrm{~cm}^{-2} D_{28}^{-2} \\
& \times(1+z)^{(p+2) / 4}(1+Y)^{-1} \\
& \times f_{p 1} \epsilon_{B,-2}^{(p-2) / 4} \epsilon_{e,-1}^{p-1} \\
& \times E_{\mathrm{K}, \mathrm{iso}, 52}^{(p+2) / 4} t_{d}^{(2-3 p) / 4} \nu_{18}^{(2-p) / 2},
\end{aligned}
$$

where $\nu f_{\nu}\left(\nu=10^{18} \mathrm{~Hz}\right.$ ) is the energy flux at $10^{18} \mathrm{~Hz}$ (in units of ergs s$\left.~^{-1} \mathrm{~cm}^{-1}\right)$, and

$$
f_{p 1}=6.73\left(\frac{p-2}{p-1}\right)^{p-1}\left(3.3 \times 10^{-6}\right)^{(p-2.3) / 2}
$$

is a function of electron spectral index $p$ (Zhang et al. 2007a). One can then derive

$$
\begin{aligned}
E_{\mathrm{K}, \text { iso }, 52}= & \left(\frac{\nu F_{\nu}\left(\nu=10^{18} \mathrm{~Hz}\right)}{5.2 \times 10^{-14} \mathrm{erg} \mathrm{s}^{-1} \mathrm{~cm}^{-2}}\right)^{4 /(p+2)} \\
& \times D_{28}^{8 /(p+2)}(1+z)^{-1}(1+Y)^{4 /(p+2)} \\
& \times f_{p 1}^{-4 /(p+2)} \epsilon_{B,-2}^{(2-p) /(p+2)} \\
& \times \epsilon_{e,-1}^{4(1-p) /(p+2)} t_{d}^{(3 p-2) /(p+2)} \nu_{18}^{2(p-2) /(p+2)},
\end{aligned}
$$



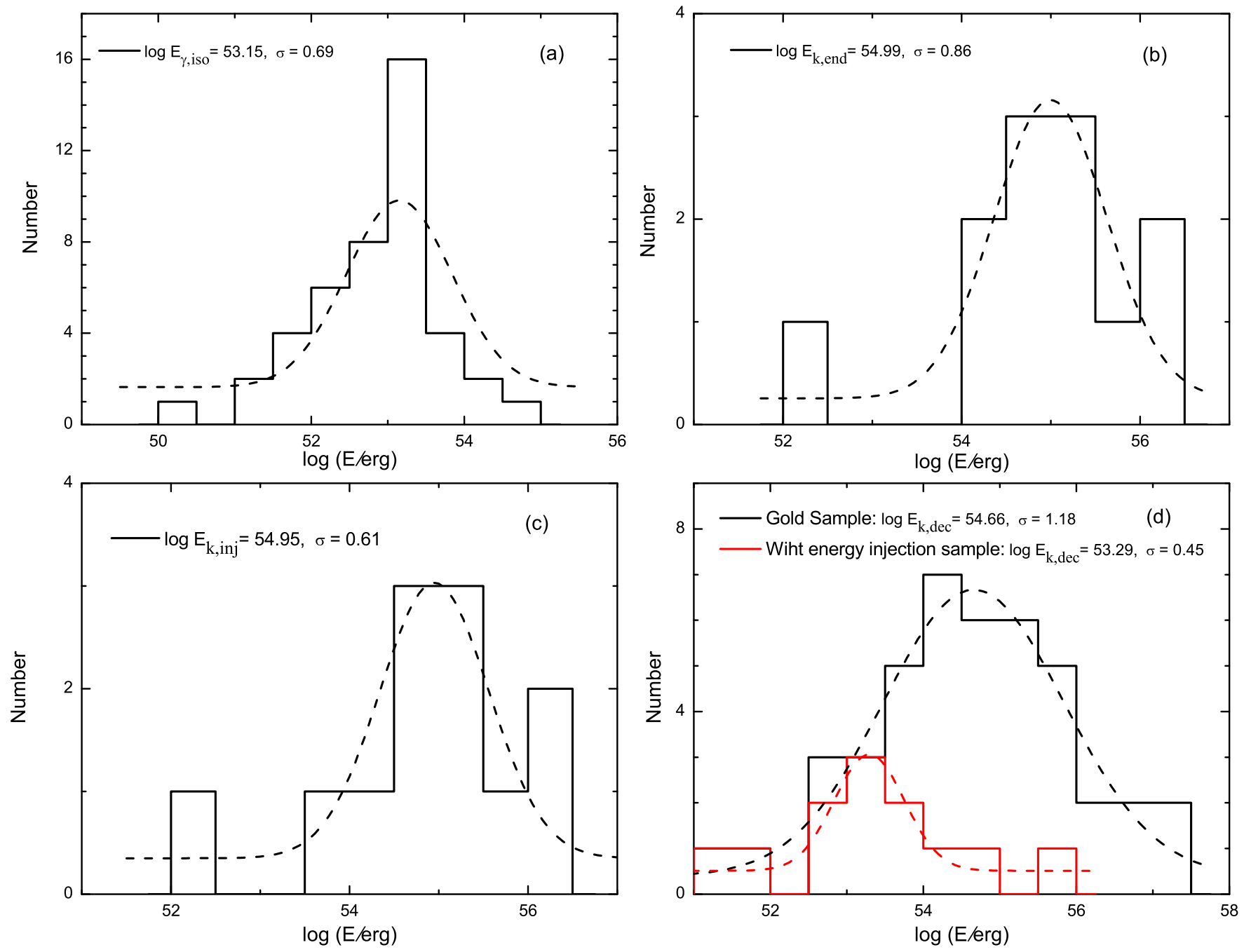

Figure 16. Distributions of various energy components derived from the Gold sample and their best Gaussian fits (dashed lines): (a) isotropic $\gamma$-ray energy, $E_{\gamma, \text { iso }}$, with a typical value of $\log \left(E_{\gamma, \text { iso }} / \mathrm{erg}\right)=(53.15 \pm 0.69)$; (b) the total isotropic kinetic energy at the end of energy injection for the energy injection sample, with a typical value of $\log \left(E_{\mathrm{K}, \text { end }} / \mathrm{erg}\right)=(54.99 \pm 0.86)$; (c) the distribution of the isotropic injected energy in the energy injection sample, with $\log \left(E_{\mathrm{K}, \mathrm{inj}} / \mathrm{erg}\right)=(54.95 \pm 0.61)$; (d) the isotropic kinetic energy at the deceleration time for the energy injection sample $\left(\log \left(E_{\mathrm{K}, \mathrm{dec}} / \mathrm{erg}\right)=(53.29 \pm 0.45)\right)$ and for the entire Gold sample $\left(\log \left(E_{\mathrm{K}, \mathrm{dec}} / \mathrm{erg}\right)=(54.66 \pm 1.18)\right)$. The following parameters are adopted in the kinetic energy calculations: $\epsilon_{e}=0.1, n=1$ or $A_{*}=1$, $Y=1$, and $\epsilon_{B}=10^{-5}$.

$$
\begin{aligned}
\epsilon_{B,-2}= & \left(\frac{6.3 \times 10^{15} \mathrm{~Hz}}{\nu_{\mathrm{c}}}\right)^{(p+2) /(p+4)} \\
& \times\left(\frac{\nu F_{\nu}\left(\nu=10^{18} \mathrm{~Hz}\right)}{5.2 \times 10^{-14} \mathrm{erg} \mathrm{s}^{-1} \mathrm{~cm}^{-2}}\right)^{-2 /(p+4)} D_{28}^{-4 /(p+4)} \\
& \times(1+Y)^{-2(p+3) /(p+4)} n^{-(p+2) /(p+4)} \\
& \times f_{p 1}^{2 /(p+4)} \epsilon_{e,-1}^{2(p-1) /(p+4)} t_{d}^{-2 p /(p+4)} \nu_{18}^{(2-p) /(p+4)}
\end{aligned}
$$

For $p>2$ and in the $\nu_{\mathrm{m}}<\nu<\nu_{\mathrm{c}}$ regime, one has

$$
\begin{aligned}
\nu F_{\nu}\left(\nu=10^{18} \mathrm{~Hz}\right)= & F_{\nu, \max } \nu_{\mathrm{m}}^{(p-1) / 2} \nu_{\mathrm{X}}^{(3-p) / 2} \\
= & 6.5 \times 10^{-13} \mathrm{erg} \mathrm{s}^{-1} \mathrm{~cm}^{-2} \\
& \times D_{28}^{-2}(1+z)^{(p+3) / 4} f_{p 1} \epsilon_{B,-2}^{(p+1) / 4} \\
& \times \epsilon_{e,-1}^{p-1} E_{\mathrm{K}, \text { iso }, 52}^{(p+3) / 4} \\
& \times n^{1 / 2} t_{d}^{(3-3 p) / 4} \nu_{18}^{(3-p) / 2},
\end{aligned}
$$

so

$$
\begin{aligned}
E_{\mathrm{K}, \text { iso }, 52}= & \left(\frac{\nu F_{\nu}\left(\nu=10^{18} \mathrm{~Hz}\right)}{6.5 \times 10^{-13} \mathrm{erg} \mathrm{s}^{-1} \mathrm{~cm}^{-2}}\right)^{4 /(p+3)} \\
& \times D_{28}^{8 /(p+3)}(1+z)^{-1} f_{p 1}^{-4 /(p+3)} \epsilon_{B,-2}^{-(p+1) /(p+3)} \\
& \times \epsilon_{e,-1}^{4(1-p) /(p+3)} n^{-2 /(p+3)} t_{d}^{(3 p-3) /(p+3)} \nu_{18}^{2(p-3) /(p+3)}, \\
\epsilon_{B,-2}= & \left(\frac{6.3 \times 10^{15} \mathrm{~Hz}}{\nu_{\mathrm{c}}}\right)^{(p+3) /(p+4)} \\
& \times\left(\frac{\nu F_{\nu}\left(\nu=10^{18} \mathrm{~Hz}\right)}{6.5 \times 10^{-13} \mathrm{erg} \mathrm{s}^{-1} \mathrm{~cm}^{-2}}\right)^{-2 /(p+4)} D_{28}^{-4 /(p+4)} \\
& \times(1+Y)^{-2(p+3) /(p+4)} n^{-(p+2) /(p+4)} \\
& \times f_{p 1}^{2 /(p+4)} \epsilon_{e,-1}^{2(p-1) /(p+4)} t_{d}^{-2 p /(p+4)} \nu_{18}^{(3-p) /(p+4)}
\end{aligned}
$$

Following Gao et al. (2013), below we derive the expressions in the $p<2$ case. 

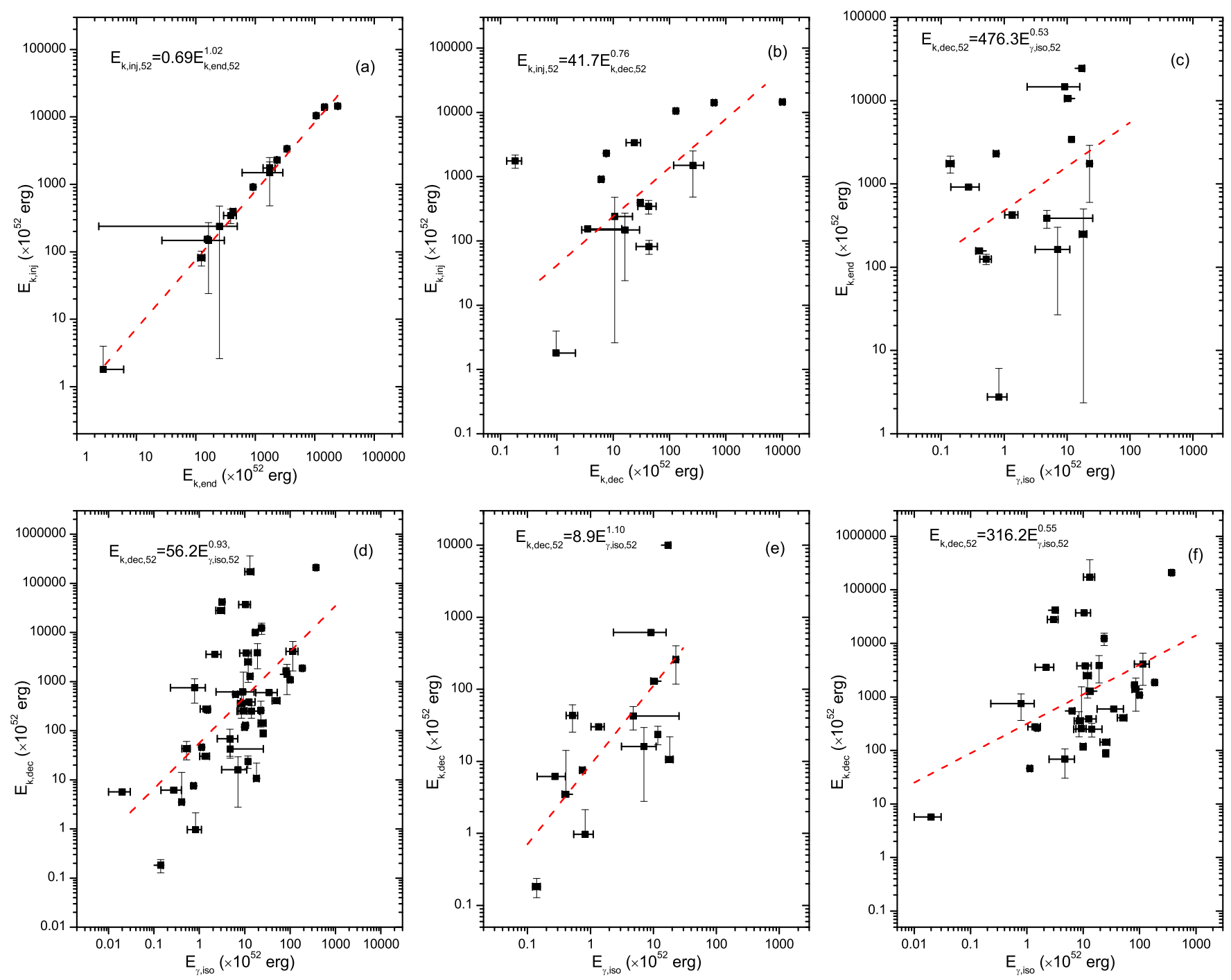

Figure 17. Correlations among different energy components: (a) $E_{\mathrm{K}, \text { end }}-E_{\mathrm{K}, \text { inj }}: E_{\mathrm{K}, \text { inj, } 52}=0.69 E_{\mathrm{K}, \text { end, }, 02}^{1.02 \pm 02}$; (b) $E_{\mathrm{K}, \text { dec }}-E_{\mathrm{K}, \text { inj }}: E_{\mathrm{K}, \text { inj, } 52}=41.7 E_{\mathrm{K}, \text { dec }, 52}^{0.76}$; (c) $E_{\gamma, \text { iso }}-E_{\mathrm{K}, \text { end }}: E_{\mathrm{K}, \text { end }, 52}=476.3 E_{\gamma, \text { iso } 52}^{0.53 \pm 0.23} ;(\mathrm{d})$, (e), and (f): $E_{\gamma, \text { iso }}-E_{\mathrm{K}, \mathrm{dec}}$ for the entire Gold sample, the energy injection subsample, and the subsample without energy injection, with $E_{\mathrm{K}, \mathrm{dec}, 52}=56.2 E_{\gamma, \mathrm{iso}, 52}^{0.93 \pm 0.19}, E_{\mathrm{K}, \mathrm{dec}, 52}=8.9 E_{\gamma, \mathrm{iso}, 52}^{1.10 \pm 0.29}$, and $E_{\mathrm{K}, \mathrm{dec}, 52}=316.2 E_{\gamma, \text { iso }, 52}^{0.55 \pm 0.21}$, respectively.

In the $\nu>\max \left(\nu_{\mathrm{m}}, \nu_{\mathrm{c}}\right)$ regime, one has

$$
\begin{aligned}
& E_{\mathrm{K}, \text { iso }}=\left(\nu F_{\nu}\left(\nu=10^{18} \mathrm{~Hz}\right)\right)^{16 /(p+14)} D^{32 /(p+14)} \\
& \times f_{p 2}^{-16 /(p+14)} n^{(p-2) /(p+14)} t^{(3 p+10) /(p+14)} \\
& \times(1+Y)^{16 /(p+14)} \\
& \times(1+z)^{-12 /(p+14)} \epsilon_{e}^{-16 /(p+14)} \nu_{18}^{8(p-2) /(p+14)}, \\
& f_{p 2}=0.00529 \times e^{0.767 p}\left(\frac{2-p}{p-1}\right) \\
& \epsilon_{B}=9.44 \times 10^{27} \nu_{\mathrm{c}}^{-2 / 3}\left(\nu F_{\nu}\left(\nu=10^{18} \mathrm{~Hz}\right)\right)^{-16 / 3(p+14)} \\
& \times D^{-32 / 3(p+14)} f_{p 2}^{16 / 3(p+14)} n^{-(3 p+26) /(3 p+42)} \\
& \times t^{-4(p+6) / 3(p+14)}(1+Y)^{-4(p+18) / 3(p+14)} \\
& \times(1+z)^{-(p+2) / 3(p+14)} \epsilon_{e}^{16 / 3(p+14)} \nu_{18}^{-8(p-2) / 3(p+14)} .
\end{aligned}
$$

In the $\nu_{\mathrm{m}}<\nu<\nu_{\mathrm{c}}$ regime, one has

$$
\begin{aligned}
& E_{\mathrm{K}, \text { iso }}=\left(\nu F_{\nu}\left(\nu=10^{18} \mathrm{~Hz}\right)\right)^{16 /(p+18)} D^{32 /(p+18)} \\
& \times f_{p 3}^{-16 /(p+18)} n^{(p-10) /(p+18)} t^{3(p+2) /(p+18)} \\
& \times(1+z)^{-16 /(p+18)} \epsilon_{e}^{-16 /(p+18)} \\
& \times \epsilon_{B}^{-12 /(p+18)} \nu_{18}^{8(p-3) /(p+18)}, \\
& \epsilon_{B}= e^{(1159.5+64.4 p) /(p+14)} \nu_{\mathrm{c}}^{-2(p+18) / 3(p+14)} \\
& \times\left(\nu F_{\nu}\left(\nu=10^{18} \mathrm{~Hz}\right)\right)^{-16 / 3(p+14)} D^{-32 / 3(p+14)} f_{p 3}^{16 / 3(p+14)} \\
& \times n^{-(3 p+26) /(3 p+42)} t^{-4(p+6) / 3(p+14)} \\
& \times(1+Y)^{-4(p+18) / 3(p+14)}(1+z)^{-(p+2) / 3(p+14)} \\
& \times \epsilon_{e}^{16 / 3(p+14)} \nu_{18}^{-8(p-3) / 3(p+14)},
\end{aligned}
$$

and

$$
f_{p 3}=5.53 \times 10^{-15} e^{0.767 p}\left(\frac{2-p}{p-1}\right) .
$$



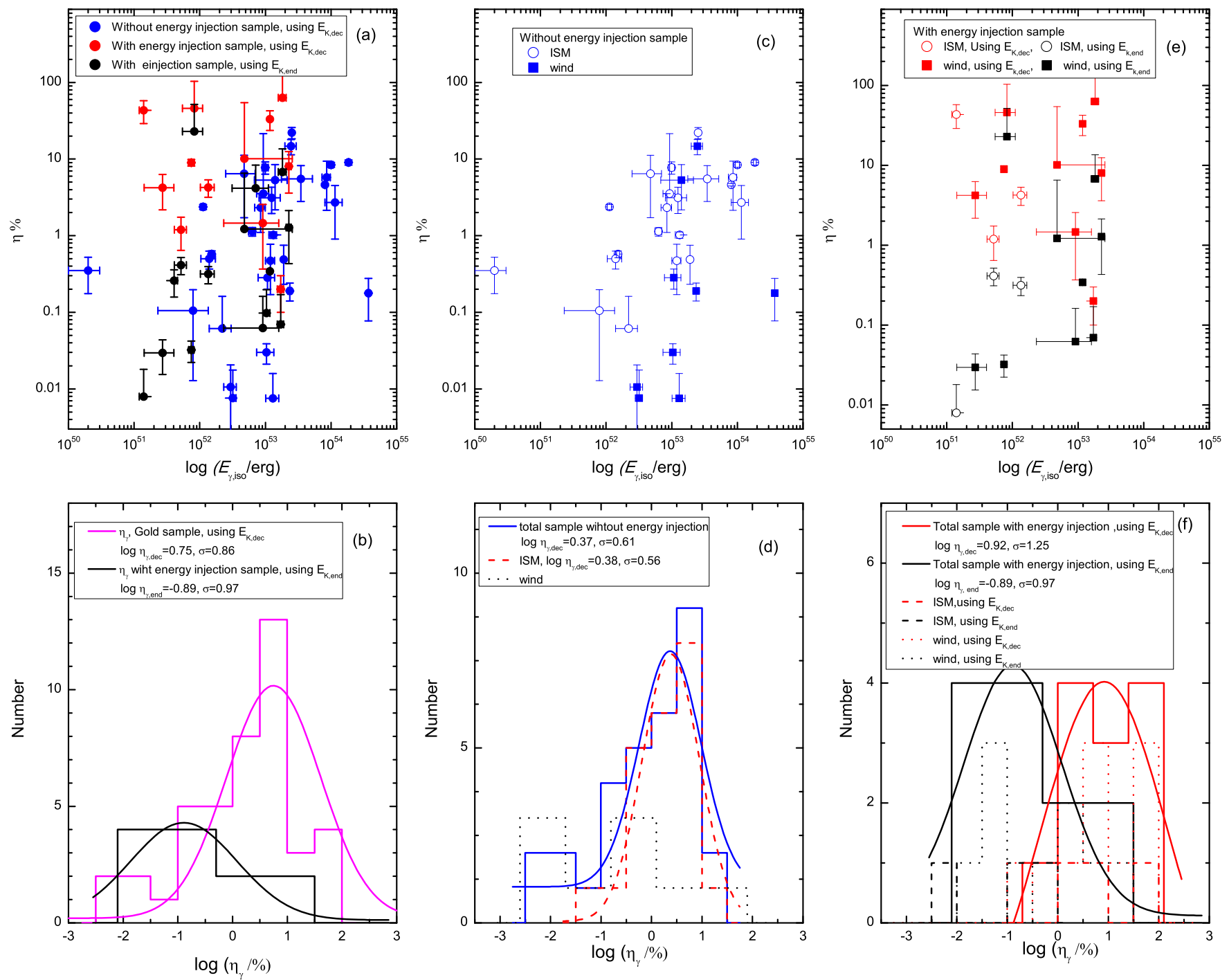

Figure 18. Radiative efficiency $\eta_{\gamma}$ of the Gold sample as a function of $E_{\gamma, \text { iso }}$ (upper row) and histograms (lower row): (a) and (b): for the entire Gold sample. Among them, the GRBs in the energy injection subsample are marked in red and black. Two efficiencies are calculated for each GRB: one using $E_{\mathrm{K}, \mathrm{dec}}$ (red) and the other using $E_{\text {end }}$ (black). Those GRBs without energy injection are marked in blue. Log-normal fits to the efficiencies derived from $E_{\mathrm{K}, \text { dec }}$ and $E_{\text {end }}$ give $\log \left(\eta_{\gamma, \mathrm{dec}} / \%\right)=0.75 \pm 0.86$ and $\log \left(\eta_{\gamma, \text { end }} / \%\right)=-0.89 \pm 0.97$, respectively. (c) and (d): for the subsample of GRBs without energy injection with best fit $\log \left(\eta_{\gamma, \mathrm{dec}} / \%\right)=0.36 \pm 0.61$. Open circles denote the ISM medium, and solid squares denote the wind medium. (e) and (f): for the subsample of GRBs with energy injection, with best fits $\log \left(\eta_{\gamma, \mathrm{dec}} / \%\right)=0.92 \pm 1.25$ and $\log \left(\eta_{\gamma, \text { end }} / \%\right)=-0.89 \pm 0.97$, respectively. Again, the ISM and wind cases are denoted as open circles and solid squares, respectively.

Table 7

Parameters of the Jet Break Sample

\begin{tabular}{lccc}
\hline \hline GRB & $\theta_{j}^{o}$ & $\log \left(E_{\gamma} / \mathrm{erg}\right)$ & $\log \left(E_{\mathrm{K}} / \mathrm{erg}\right)$ \\
\hline $050820 \mathrm{~A}$ & $4.5 \pm 3.0$ & $51.54_{-0.57}^{+0.24}$ & $53.05_{-0.99}^{+0.28}$ \\
$050922 \mathrm{C}$ & $1.8 \pm 0.3$ & $49.69_{-0.10}^{+0.08}$ & $50.13_{-0.12}^{+0.09}$ \\
$060111 \mathrm{~B}^{\mathrm{a}}$ & $\ldots$ & $\ldots$ & $\ldots$ \\
081008 & $1.3 \pm 0.4$ & $49.41_{-0.21}^{+0.14}$ & $50.54_{-0.29}^{+0.17}$ \\
$081203 \mathrm{~A}$ & $1.0 \pm 0.6$ & $49.76_{-0.56}^{+0.24}$ & $50.72_{-0.33}^{+0.19}$ \\
090618 & $3.5 \pm 1.3$ & $50.68_{-0.21}^{+0.14}$ & $50.84_{-0.23}^{+0.15}$ \\
091127 & $2.7 \pm 0.4$ & $49.22_{-0.08}^{+0.07}$ & $50.73_{-0.09}^{+0.07}$ \\
$130427 \mathrm{~A}$ & $3.8 \pm 0.3$ & $51.25_{-0.04}^{+0.04}$ & $51.54_{-0.04}^{+0.04}$ \\
\hline
\end{tabular}

Note.

${ }^{\mathrm{a}}$ No redshift $z$ available.

\section{A.2. The Wind Model}

The following derivations follow Chevalier \& $\mathrm{Li}$ (2000), Gao et al. (2013), and Lü \& Zhang (2014).

For the $p>2$ case, the forward shock emission can be characterized as

$$
\begin{aligned}
\nu_{\mathrm{m}}= & 5.2 \times 10^{11} \mathrm{~Hz}\left(\frac{p-2}{p-1}\right)^{2} \\
& \times(1+z)^{1 / 2} \epsilon_{B,-2}^{1 / 2} \epsilon_{e,-1}^{2} E_{\mathrm{K}, \text { iso }, 52}^{1 / 2} t_{d}^{-3 / 2}, \\
\nu_{\mathrm{c}}= & 1.7 \times 10^{18} \mathrm{~Hz}(1+z)^{-3 / 2} \\
& \times(1+Y)^{-2} \epsilon_{B,-2}^{-3 / 2} E_{\mathrm{K}, \mathrm{iso}, 52}^{1 / 2} A_{*,-1}^{-2} t_{d}^{1 / 2}, \\
F_{\nu, \max }= & 1.6 \mathrm{mJy}(1+z)^{3 / 2} D_{28}^{-2} \epsilon_{B,-2}^{1 / 2} E_{\mathrm{K}, \text { iso }, 52}^{1 / 2} A_{*,-1} t_{d}^{-1 / 2},
\end{aligned}
$$



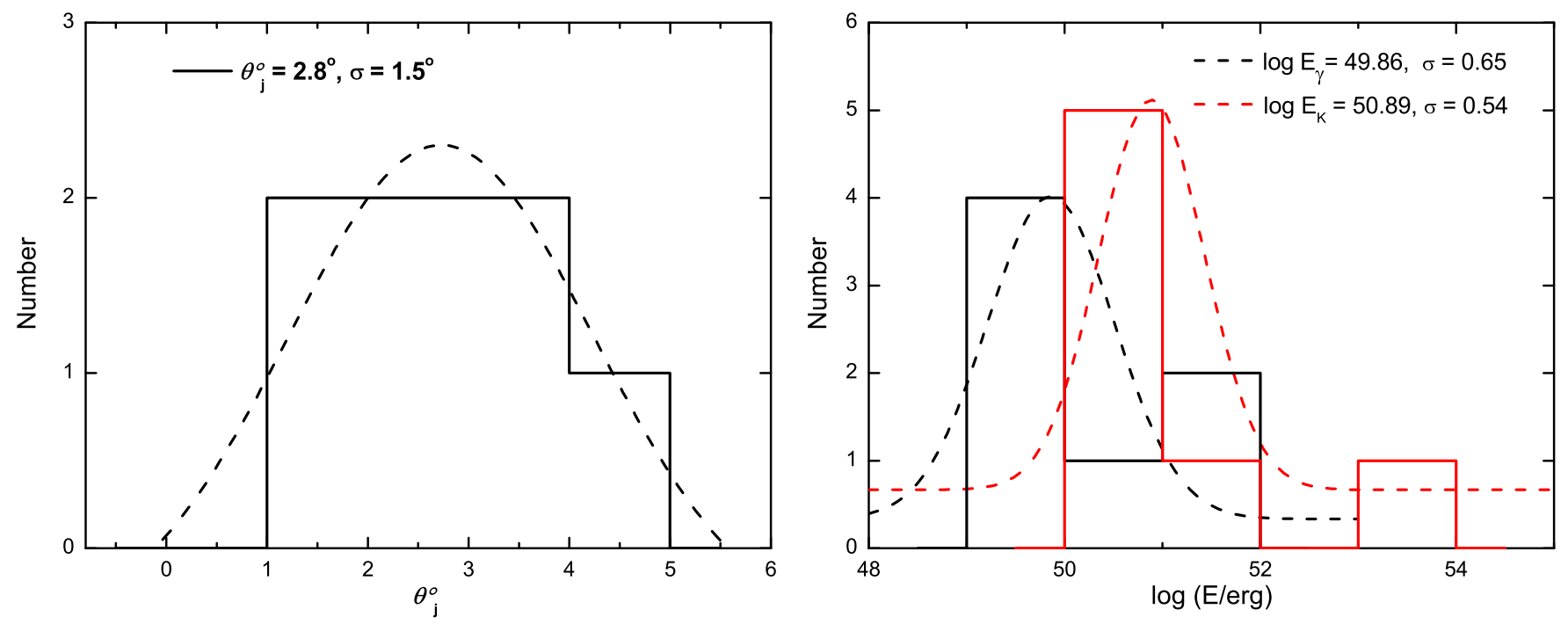

Figure 19. The distributions of the jet opening angle $\left(\theta_{j}\right)$, geometrically corrected $\gamma$-ray energy $\left(E_{\gamma}\right)$, and kinetic energy $\left(E_{\mathrm{K}}\right)$, respectively, derived from the jet break subsample of the Gold sample. The dashed lines are the best Gaussian fits, with $\theta_{j}=(2.8 \pm 1.5) \log \left(E_{\gamma} / \mathrm{erg}\right)=(49.86 \pm 0.65)$ and $\log \left(E_{\mathrm{K}} / \mathrm{erg}\right)=(50.89 \pm 0.54)$, respectively.

where $A_{*}$ is the density parameter of the stellar wind medium.

For $p>2$ and in the $\nu>\max \left(\nu_{\mathrm{m}}, \nu_{\mathrm{c}}\right)$ regime, one has

$$
\begin{aligned}
& \nu F_{\nu}\left(\nu=10^{18} \mathrm{~Hz}\right)=F_{\nu, \max } \nu_{\mathrm{c}}^{1 / 2} \nu_{\mathrm{m}}^{(p-1) / 2} \nu_{\mathrm{X}}^{(2-p) / 2} \\
& =2.6 \times 10^{-13} \mathrm{erg} \mathrm{s}^{-1} \mathrm{~cm}^{-2} D_{28}^{-2} \\
& \times(1+z)^{(p+2) / 4}(1+Y)^{-1} \\
& \times f_{p 4} \epsilon_{B,-2}^{(p-2) / 4} \epsilon_{e,-1}^{p-1} \\
& \times E_{\mathrm{K}, \mathrm{is}, 52}^{(p+2) / 4} t_{d}^{(2-3 p) / 4} \nu_{18}^{(2-p) / 2} \text {, } \\
& f_{p 4}=6.73\left(\frac{p-2}{p-1}\right)^{p-1}\left(5.2 \times 10^{-7}\right)^{(p-2.3) / 2} \\
& E_{\mathrm{K}, \text { iso }, 52}=\left(\frac{\nu F_{\nu}\left(\nu=10^{18} \mathrm{~Hz}\right)}{2.6 \times 10^{-13} \mathrm{erg} \mathrm{s}^{-1} \mathrm{~cm}^{-2}}\right)^{4 /(p+2)} \\
& \times D_{28}^{8 /(p+2)}(1+z)^{-1}(1+Y)^{4 /(p+2)} \\
& \times f_{p 4}^{-4 /(p+2)} \epsilon_{B,-2}^{(2-p) /(p+2)} \\
& \times \epsilon_{e,-1}^{4(1-p) /(p+2)} t_{d}^{(3 p-2) /(p+2)} \nu_{18}^{2(p-2) /(p+2)}, \\
& \epsilon_{B,-2}=\left(\frac{1.7 \times 10^{18} \mathrm{~Hz}}{\nu_{\mathrm{c}}}\right)^{(p+2) /(2 p+2)} \\
& \times\left(\frac{\nu F_{\nu}\left(\nu=10^{18} \mathrm{~Hz}\right)}{2.6 \times 10^{-13} \mathrm{erg} \mathrm{s}^{-1} \mathrm{~cm}^{-2}}\right)^{1 /(p+1)} \\
& \times D_{28}^{2 /(p+1)}(1+z)^{-(p+2) /(p+1)} \\
& \times(1+Y)^{-1} f_{p 4}^{-1 /(p+1)} \epsilon_{e,-1}^{(1-p) /(p+1)} \\
& \times A_{*,-1}^{-(p+2) /(p+1)} t_{d}^{p /(p+1)} \nu_{18}^{(p-2) / 2(p+1)} .
\end{aligned}
$$

For $p>2$ and in the $\nu_{\mathrm{m}}<\nu<\nu_{\mathrm{c}}$ regime, one has

$$
\begin{aligned}
\nu F_{\nu}\left(\nu=10^{18} \mathrm{~Hz}\right)= & F_{\nu, \max } \nu_{\mathrm{m}}^{(p-1) / 2} \nu_{\mathrm{X}}^{(3-p) / 2} \\
= & 2.0 \times 10^{-13} \mathrm{erg} \mathrm{s}^{-1} \mathrm{~cm}^{-2} D_{28}^{-2} \\
& \times(1+z)^{(p+5) / 4} f_{p 4} \epsilon_{B,-2}^{(+1) / 4} \epsilon_{e,-1}^{p-1} E_{\mathrm{K}, \text { iso,52 }}^{(p+1) / 4} \\
& \times A_{*,-1} t_{d}^{(1-3 p) / 4} \nu_{18}^{(3-p) / 2}, \\
E_{\mathrm{K}, \text { iso, } 52}= & \left(\frac{\nu F_{\nu}\left(\nu=10^{18} \mathrm{~Hz}\right)}{2.0 \times 10^{-13} \mathrm{erg} \mathrm{s}^{-1} \mathrm{~cm}^{-2}}\right)^{4 /(p+1)} \\
\times & D_{28}^{8 /(p+1)}(1+z)^{-(p+5) /(p+1)} f_{p 4}^{-4 /(p+1)} \epsilon_{B,-2}^{-1} \\
\times & \epsilon_{e,-1}^{4(1-p) /(p+1)} A_{*,-1}^{-4 /(p+1)} t_{d}^{(3 p-1) /(p+1)} \nu_{18}^{2(p-3) /(p+1)}, \\
\epsilon_{B,-2}= & \left(\frac{1.7 \times 10^{18} \mathrm{~Hz}}{\nu_{\mathrm{c}}}\right)^{1 / 2} \\
& \times\left(\frac{\nu F_{\nu}\left(\nu=10^{18} \mathrm{~Hz}\right)}{2.0 \times 10^{-13} \mathrm{erg} \mathrm{s}^{-1} \mathrm{~cm}^{-2}}\right)^{1 /(p+1)} \\
& \times D_{28}^{2 /(p+1)}(1+z)^{-(p+2) /(p+1)} \\
& \times(1+Y)^{-1} A_{*,-1}^{-(p+2) /(p+1)} f_{p 4}^{-1 /(p+1)} \\
& \times \epsilon_{e,-1}^{(1-p) /(p+1)} t_{d}^{p /(p+1)} \nu_{18}^{(p-3) / 2(p+1)} .
\end{aligned}
$$

For $p<2$ and in the $\nu>\max \left(\nu_{\mathrm{m}}, \nu_{\mathrm{c}}\right)$ regime, one has

$$
\begin{aligned}
E_{\mathrm{K}, \text { iso }}= & \left(\nu F_{\nu}\left(\nu=10^{18} \mathrm{~Hz}\right)\right)^{8 /(p+6)} D^{16 /(p+6)} \\
& \times f_{p 5}^{-8 /(p+6)} A^{(p-2) /(p+6)} t \\
& \times(1+Y)^{8 /(p+6)} \times(1+z)^{-6 /(p+6)} \\
& \times \epsilon_{e}^{-8 /(p+6)} \nu_{18}^{4(p-2) /(p+6)},
\end{aligned}
$$




$$
\begin{aligned}
f_{p 5} & =405854 \times 3^{3 p / 8} 5^{-p / 4} e^{-7 p} \pi^{(4-3 p) / 8}\left(\frac{2-p}{p-1}\right), \\
\epsilon_{B}= & 6.87 \times 10^{-11} \nu_{\mathrm{c}}^{-2 / 3}\left(\nu F_{\nu}\left(\nu=10^{18} \mathrm{~Hz}\right)\right)^{8 / 3(p+6)} \\
& \times D^{16 / 3(p+6)} f_{p 5}^{-8 / 3(p+6)} A^{-(3 p+26) /(3 p+18)} t^{2 / 3} \\
& \times(1+Y)^{-4(p+4) / 3(p+6)}(1+z)^{-(p+8) /(p+6)} \\
& \times \epsilon_{e}^{-8 / 3(p+6)} \nu_{18}^{4(p-2) / 3(p+6)} .
\end{aligned}
$$

For $p<2$ and in the $\nu_{\mathrm{m}}<\nu<\nu_{\mathrm{c}}$ regime, one has

$$
\begin{aligned}
E_{\mathrm{K}, \text { iso }}= & \left(\nu F_{\nu}\left(\nu=10^{18} \mathrm{~Hz}\right)\right)^{8 /(p+4)} D^{16 /(p+4)} \\
& \times f_{p 6}^{-8 /(p+4)} A^{(p-10) /(p+4)} t^{(p+8) /(p+4)} \\
& \times(1+z)^{-12 /(p+4)} \epsilon_{e}^{-8 /(p+4)} \epsilon_{B}^{-6 /(p+4)} \nu_{18}^{4(p-3) /(p+4)}, \\
& f_{p 6}=1.72 \times 10^{22} \times 2^{-17 p / 2} 3^{3 p / 8} 5^{-37 p / 4} \\
& \quad \times e^{13.38 p} \pi^{(4-3 p) / 8}\left(\frac{2-p}{p-1}\right)
\end{aligned}
$$

and

$$
\begin{aligned}
\epsilon_{B}= & \left(5.69 \times 10^{-16}\right)^{2(p+4) / 3(p+6)} \nu_{\mathrm{c}}^{-2(p+4) / 3(p+6)} \\
& \times\left(\nu F_{\nu}\left(\nu=10^{18} \mathrm{~Hz}\right)\right)^{8 / 3(p+6)} D^{16 / 3(p+6)} f_{p 6}^{-8 / 3(p+6)} \\
& \times A^{-(3 p+26) /(3 p+18)} t^{2 / 3}(1+Y)^{-4(p+4) / 3(p+6)} \\
& \times(1+z)^{-(p+8) /(p+6)} \epsilon_{e}^{-8 / 3(p+6)} \nu_{18}^{4(p-3) / 3(p+6)}
\end{aligned}
$$

\section{REFERENCES}

Achterberg, A., Gallant, Y. A., Kirk, J. G., \& Guthmann, A. W. 2001, MNRAS, 328, 393

Band, D., Matteson, J., Ford, L., et al. 1993, ApJ, 413, 281

Barniol Duran, R. 2014, MNRAS, 442, 3147

Barthelmy, S. D., Cannizzo, J. K., Gehrels, N., et al. 2005, ApJL, 635, L133

Beloborodov, A. M. 2000, ApJL, 539, L25

Berger, E., Kulkarni, S. R., Pooley, G., et al. 2003, Natur, 426, 154

Bloom, J. S., Frail, D. A., \& Sari, R. 2001, AJ, 121, 2879

Burrows, D. N., Romano, P., Falcone, A., et al. 2005, Sci, 309, 1833

Chevalier, R. A., \& Li, Z.-Y. 2000, ApJ, 536, 195

Chincarini, G., Moretti, A., Romano, P., et al. 2007, ApJ, 671, 1903

Costa, E., Frontera, F., Heise, J., et al. 1997, Natur, 387, 783

Curran, P. A., Evans, P. A., De Pasquale, M., Page, M. J., \& van der Horst, A. J. 2010, ApJL, 716, L135

Dai, Z. G., \& Lu, T. 1998, A\&A, 333, L87

Deng, W., Li, H., Zhang, B., \& Li, S. 2015, ApJ, 805, 163

De Pasquale, M., Evans, P., Oates, S., et al. 2009, MNRAS, 392, 153

Ellison, D. C., \& Double, G. P. 2002, APh, 18, 213

Fan, Y., \& Piran, T. 2006, MNRAS, 369, 197

Fan, Y. Z., \& Wei, D. M. 2005, MNRAS, 364, L42

Frail, D. A., Kulkarni, S. R., Nicastro, L., Feroci, M., \& Taylor, G. B. 1997, Natur, 389, 261

Frail, D. A., Kulkarni, S. R., Sari, R., et al. 2001, ApJL, 562, L55

Freedman, D. L., \& Waxman, E. 2001, ApJ, 547, 922

Gao, H., Lei, W.-H., Zou, Y.-C., Wu, X.-F., \& Zhang, B. 2013, NewAR, 57,141

Gao, H., \& Mészáros, P. 2015, ApJ, 802, 90

Gao, H., \& Zhang, B. 2015, ApJ, 801, 103

Gehrels, N., Chincarini, G., Giommi, P., et al. 2004, ApJ, 611, 1005
Genet, F., Daigne, F., \& Mochkovitch, R. 2007, MNRAS, 381, 732

Ghisellini, G., Ghirlanda, G., Nava, L., \& Firmani, C. 2007, ApJL, 658, L75

Granot, J., \& Kumar, P. 2003, ApJ, 591, 1086

Granot, J., \& Sari, R. 2002, ApJ, 568, 820

Harrison, F. A., Bloom, J. S., Frail, D. A., et al. 1999, ApJL, 523, L121

Huang, K. Y., Urata, Y., Kuo, P. H., et al. 2007, ApJL, 654, L25

Huang, Y. F., Dai, Z. G., \& Lu, T. 1999, MNRAS, 309, 513

Huang, Y. F., Gou, L. J., Dai, Z. G., \& Lu, T. 2000, ApJ, 543, 90

Ioka, K., Kobayashi, S., \& Zhang, B. 2005, ApJ, 631, 429

Kaneko, Y., Preece, R. D., Briggs, M. S., et al. 2006, ApJS, 166, 298

Kann, D. A., Klose, S., \& Zeh, A. 2006, ApJ, 641, 993

Kann, D. A., Klose, S., Zhang, B., et al. 2010, ApJ, 720, 1513

Kann, D. A., Klose, S., Zhang, B., et al. 2011, ApJ, 734, 96

Kobayashi, S., \& Sari, R. 2001, ApJ, 551, 934

Kumar, P. 1999, ApJL, 523, L113

Kumar, P., \& Barniol Duran, R. 2009, MNRAS, 400, L75

Kumar, P., \& Barniol Duran, R. 2010, MNRAS, 409, 226

Kumar, P., \& Granot, J. 2003, ApJ, 591, 1075

Kumar, P., Narayan, R., \& Johnson, J. L. 2008a, MNRAS, 388, 1729

Kumar, P., Narayan, R., \& Johnson, J. L. 2008b, Sci, 321, 376

Kumar, P., \& Zhang, B. 2015, PhR, 561, 1

Lazzati, D., Morsony, B. J., Margutti, R., \& Begelman, M. C. 2013, ApJ, 765,103

Lazzati, D., \& Perna, R. 2007, MNRAS, 375, L46

Li, L., Liang, E.-W., Tang, Q.-W., et al. 2012, ApJ, 758, 27

Li, L., Wu, X.-F., Huang, Y.-F., et al. 2015, ApJ, 805, 13

Liang, E.-W., Racusin, J. L., Zhang, B., Zhang, B.-B., \& Burrows, D. N. 2008 , $\mathrm{ApJ}, 675,528$

Liang, E.-W., Zhang, B.-B., \& Zhang, B. 2007, ApJ, 670, 565

Liang, E. W., Zhang, B., O'Brien, P. T., et al. 2006, ApJ, 646, 351

Liang, E.-W., Li, L., Gao, H., et al. 2013, ApJ, 774, 13

Lloyd-Ronning, N. M., \& Zhang, B. 2004, ApJ, 613, 477

Lü, H.-J., \& Zhang, B. 2014, ApJ, 785, 74

Margutti, R., Guidorzi, C., Chincarini, G., et al. 2010, MNRAS, 406, 2149

Maselli, A., Melandri, A., Nava, L., et al. 2014, Sci, 343, 48

Maxham, A., \& Zhang, B. 2009, ApJ, 707, 1623

Medvedev, M. V., \& Loeb, A. 1999, ApJ, 526, 697

Mészáros, P., \& Rees, M. J. 1997, ApJ, 476, 232

Mészáros, P., Rees, M. J., \& Wijers, R. A. M. J. 1998, ApJ, 499, 301

Molinari, E., Vergani, S. D., Malesani, D., et al. 2007, A\&A, 469, L13

Nardini, M., Ghisellini, G., Ghirlanda, G., et al. 2006, A\&A, 451, 821

Nishikawa, K.-I., Niemiec, J., Hardee, P. E., et al. 2009, ApJL, 698, L10

Nousek, J. A., Kouveliotou, C., Grupe, D., et al. 2006, ApJ, 642, 389

Panaitescu, A., \& Kumar, P. 2001, ApJ, 554, 667

Panaitescu, A., \& Kumar, P. 2002, ApJ, 571, 779

Panaitescu, A., Mészáros, P., Gehrels, N., Burrows, D., \& Nousek, J. 2006, MNRAS, 366, 1357

Panaitescu, A., Mészáros, P., \& Rees, M. J. 1998, ApJ, 503, 314

Panaitescu, A., Spada, M., \& Mészáros, P. 1999, ApJL, 522, L105

Panaitescu, A., \& Vestrand, W. T. 2008, MNRAS, 387, 497

Panaitescu, A., \& Vestrand, W. T. 2011, MNRAS, 414, 3537

Perley, D. A., Cenko, S. B., Corsi, A., et al. 2014, ApJ, 781, 37

Preece, R. D., Briggs, M. S., Mallozzi, R. S., et al. 2000, ApJS, 126, 19

Racusin, J. L., Liang, E. W., Burrows, D. N., et al. 2009, ApJ, 698, 43

Racusin, J. L., Oates, S. R., Schady, P., et al. 2011, ApJ, 738, 138

Rees, M. J., \& Mészáros, P. 1998, ApJ, 496, L1

Rhoads, J. E. 1999, ApJ, 525, 737

Rossi, E., Lazzati, D., \& Rees, M. J. 2002, MNRAS, 332, 945

Sakamoto, T., Sato, G., Barbier, L., et al. 2009, ApJ, 693, 922

Santana, R., Barniol Duran, R., \& Kumar, P. 2014, ApJ, 785, 29

Sari, R., \& Esin, A. A. 2001, ApJ, 548, 787

Sari, R., \& Mészáros, P. 2000, ApJL, 535, L33

Sari, R., Piran, T., \& Halpern, J. P. 1999, ApJL, 519, L17

Sari, R., Piran, T., \& Narayan, R. 1998, ApJL, 497, L17

Schlegel, D. J., Fink beiner, D. P., \& Davis, M. 1998, ApJ, 500, 525

Schulze, S., Klose, S., Björnsson, G., et al. 2011, A\&A, 526, A23

Shao, L., \& Dai, Z. G. 2007, ApJ, 660, 1319

Shen, R., Kumar, P., \& Robinson, E. L. 2006, MNRAS, 371, 1441

Swenson, C. A., Roming, P. W. A., De Pasquale, M., \& Oates, S. R. 2013, ApJ, 774, 2

Tagliaferri, G., Goad, M., Chincarini, G., et al. 2005, Natur, 436, 985

Troja, E., Cusumano, G., O’Brien, P. T., et al. 2007, ApJ, 665, 599

Uhm, Z. L., \& Beloborodov, A. M. 2007, ApJL, 665, L93

Uhm, Z. L., \& Zhang, B. 2014a, ApJ, 780, 82

Uhm, Z. L., \& Zhang, B. 2014b, ApJ, 789, 39 
Uhm, Z. L., \& Zhang, B. 2014c, NatPh, 10, 351

Uhm, Z. L., Zhang, B., Hascoët, R., et al. 2012, ApJ, 761, 147

van Eerten, H. J., \& Wijers, R. A. M. J. 2009, MNRAS, 394, 2164

van Paradijs, J., Groot, P. J., Galama, T., et al. 1997, Natur, 386, 686

Virgili, F. J., Qin, Y., Zhang, B., \& Liang, E. 2012, MNRAS, 424, 2821

Wang, X.-G., Liang, E.-W., Li, L., et al. 2013, ApJ, 774, 132

Waxman, E. 1997, ApJ, 485, L5

Willingale, R., O'Brien, P. T., Osborne, J. P., et al. 2007, ApJ, 662, 1093

Wijers, R. A. M. J., \& Galama, T. J. 1999, ApJ, 523, 177

Wijers, R. A. M. J., Rees, M. J., \& Meszaros, P. 1997, MNRAS, 288, L51

Wu, X. F., Dai, Z. G., \& Liang, E. W. 2004, ApJ, 615, 359

Yi, S.-X., Wu, X.-F., \& Dai, Z.-G. 2013, ApJ, 776, 120
Yost, S. A., Harrison, F. A., Sari, R., \& Frail, D. A. 2003, ApJ, 597, 459

Zhang, B., Fan, Y. Z., Dyks, J., et al. 2006, ApJ, 642, 354

Zhang, B., Liang, E., Page, K. L., et al. 2007a, ApJ, 655, 989

Zhang, B., \& Mészáros, P. 2001, ApJL, 552, L35

Zhang, B., \& Mészáros, P. 2002, ApJ, 566, 712

Zhang, B., \& Mészáros, P. 2004, IJMPA, 19, 2385

Zhang, B., \& Yan, H. 2011, ApJ, 726, 90

Zhang, B., Zhang, B.-B., Liang, E.-W., et al. 2007b, ApJL, 655, L25

Zhang, B.-B., Liang, E.-W., \& Zhang, B. 2007c, ApJ, 666, 1002

Zhang, B.-B., Zhang, B., Murase, K., Connaughton, V., \& Briggs, M. S. 2014 ApJ, 787, 66

Zhang, W., \& MacFadyen, A. 2009, ApJ, 698, 1261 